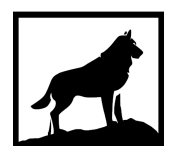

Michigan Technological

1 8 8 5 University
Michigan Technological University Digital Commons @ Michigan Tech

\title{
A STUDY OF MODEL-BASED CONTROL STRATEGY FOR A GASOLINE TURBOCHARGED DIRECT INJECTION SPARK IGNITED ENGINE
}

Xin Wang

Michigan Technological University, xwang15@mtu.edu

Copyright 2020 Xin Wang

\section{Recommended Citation}

Wang, Xin, "A STUDY OF MODEL-BASED CONTROL STRATEGY FOR A GASOLINE TURBOCHARGED DIRECT INJECTION SPARK IGNITED ENGINE", Open Access Dissertation, Michigan Technological University, 2020.

https://doi.org/10.37099/mtu.dc.etdr/1116

Follow this and additional works at: https://digitalcommons.mtu.edu/etdr

Part of the Automotive Engineering Commons, Computer-Aided Engineering and Design Commons, and the Controls and Control Theory Commons 
A STUDY OF MODEL-BASED CONTROL STRATEGY FOR A GASOLINE

TURBOCHARGED DIRECT INJECTION SPARK IGNITED ENGINE

\author{
By \\ Xin Wang

\begin{abstract}
A DISSERTATION
Submitted in partial fulfillment of the requirements for the degree of DOCTOR OF PHILOSOPHY

In Mechanical Engineering - Engineering Mechanics
\end{abstract}

MICHIGAN TECHNOLOGICAL UNIVERSITY

2020

(C) 2020 Xin Wang 
This dissertation has been approved in partial fulfillment of the requirements for the Degree of DOCTOR OF PHILOSOPHY in Mechanical Engineering - Engineering Mechanics.

Department of Mechanical Engineering - Engineering Mechanics

Dissertation Co-Advisor: $\quad$ Dr. Bo Chen

Dissertation Co-Advisor: $\quad$ Dr. Jeffrey D. Naber

Committee Member: Dr. Mahdi Shahbakhti

Committee Member: $\quad$ Dr. David D. Wanless

Department Chair: Dr. William Predebon 


\section{Table of Contents}

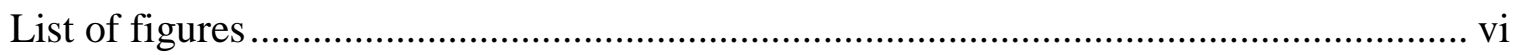

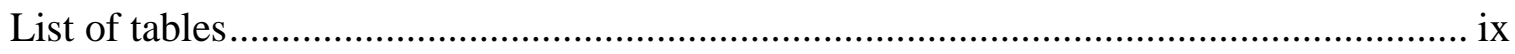

Preface

Acknowledgements ................................................................................................... xii

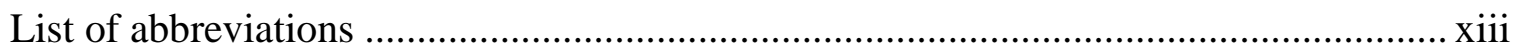

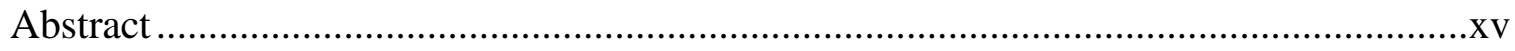

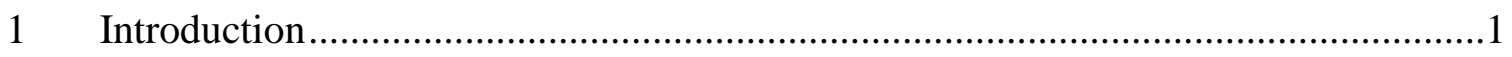

$1.1 \quad$ Background and Research Motivation …………......................................

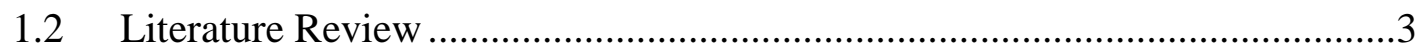

1.2.1 GTDI SI Engine Control Problems and Approaches ............................

1.2.2 Combustion Phasing Control ...........................................................

1.2.3 Model Predictive Control and its Applications in Automotive

Control Problems...................................................................................10

1.2.3.1 Model Predictive Control................................................10

1.2.3.2 Applications of MPC for Automotive Control ...............12

1.2.3.3 MPC-Based Engine Control ...........................................13

1.3 Research Objectives and Tasks ……………….........................................

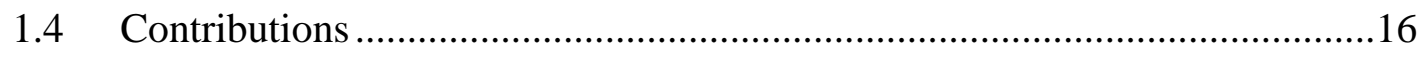

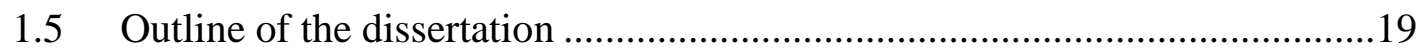

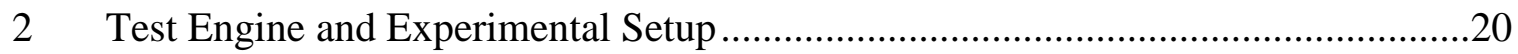

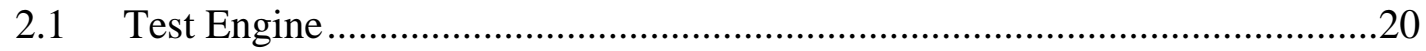

2.2 Experimental Setup ………………………….................................22

3 Model-Based Combustion Duration and Ignition Timing Prediction for Combustion Phasing Control of a Spark-Ignition Engine Using In-Cylinder Pressure Sensors [108] ..24

3.1 Overview of Model-Based Burn Duration and Ignition Timing Management for Combustion Phasing Control ...........................................................................24

3.2 SI Combustion Modeling for Engine Control ...............................................26

3.3 Control-Oriented Combustion Model Development......................................27

3.3.1 Flame Development Stage Model..................................................28

3.3.2 Rapid Burning Stage Model.................................................................

3.3.3 Dynamic Burn Duration and Ignition Timing Prediction ...................39 


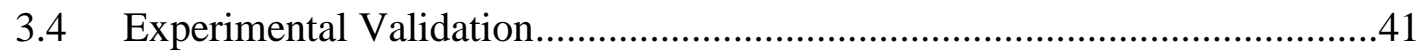

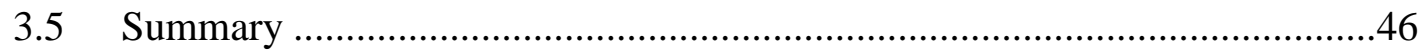

4 Adaptive Ignition Timing Management and Feedback Combustion Phasing Estimation for Combustion Phasing Control ................................................................47

4.1 Adaptive Model-Based Ignition Timing Management .................................47

4.1.1 Overview of Adaptive Model-Based Ignition Timing Management 48

4.1.2 Recursive Least Square-Based Model Adaptation ............................51

4.1.3 RLS-Based Model Adaptation Experimental Validation ...................57

4.2 Adaptive Model-Based Combustion Phasing Estimation ................................62

4.2.1 AEKF-based Feedback Combustion Phasing Estimation ...................63

4.2.2 Experimental Validation of AEKF-based Burn Duration and CA50

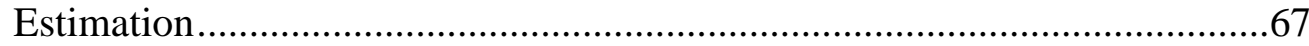

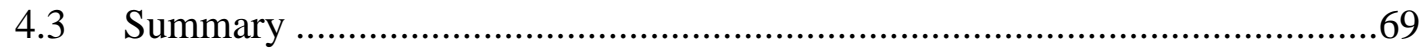

5 The Economic Nonlinear Model Predictive Control of GTDI SI Engine .................70

5.1 Overview of Cycle-by-Cycle GTDI SI Engine Control using E-NMPC ........71

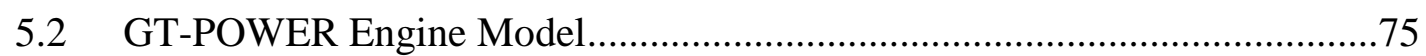

5.2.1 GT-POWER Engine Model Development......................................75

5.2.2 Calibration and Validation of the GT-POWER Engine Model .........77

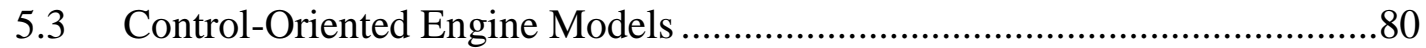

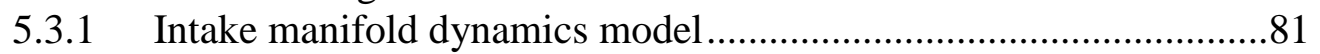

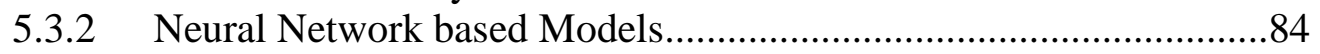

5.3.2.1 Volumetric Efficiency Model ..........................................89

5.3.2.2 Throttle inlet pressure model ...........................................89

5.3.2.3 IMEPn Model...........................................................93

5.3.2.4 NOx Model ...............................................................

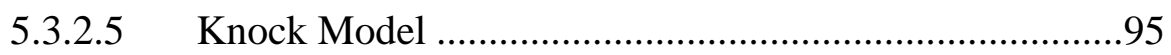

5.3.2.6 COV of IMEP Model ...................................................96

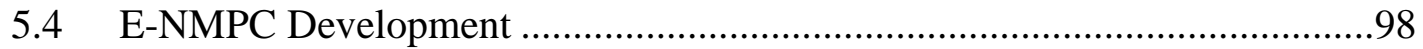

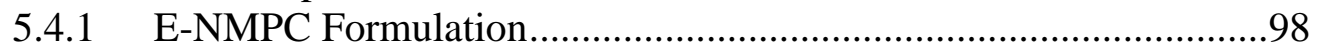

5.4.2 E-NMPC Implementation for SIL Co-simulation.............................101

5.5 Performance Assessment with SIL Co-simulation.......................................103

5.5.1 Transient Test Assessment...........................................................103

5.5.2 Economic Performances Validation ................................................106

5.5.2.1 VVT Control Evaluation.............................................106

5.5.2.2 CA50 Control Evaluation ..........................................109

5.5.3 Real World Driving Cycle Assessment …………..........................110

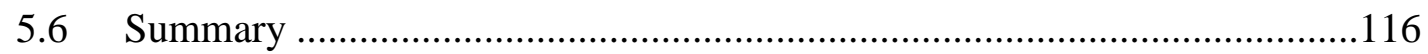




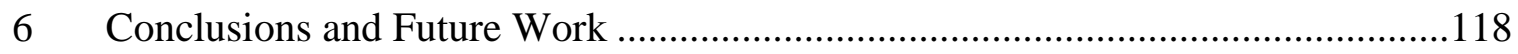

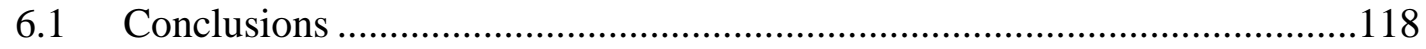

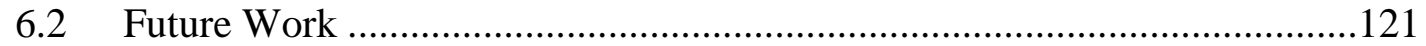

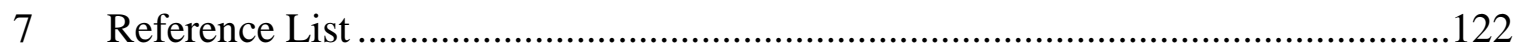

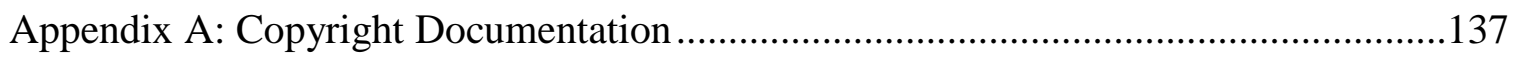




\section{List of figures}

Figure 1.1 Light-duty Vehicle Stock [1] ...................................................................

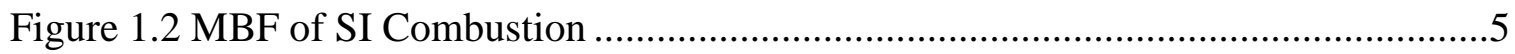

Figure 1.3 Influence of Combustion Phasing (CA50) on Engine Efficiency [28]...............6

Figure 1.4 An Example of Application of E-NMPC based Engine Control......................11

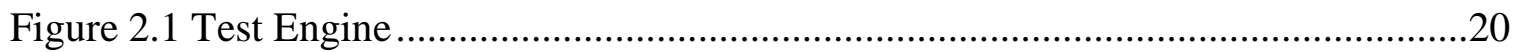

Figure 2.2 Schematic of Experimental 2.0L GTDI engine ..............................................23

Figure 3.1 Structure of Model-Based Combustion Phasing Control in GTDI SI Engine

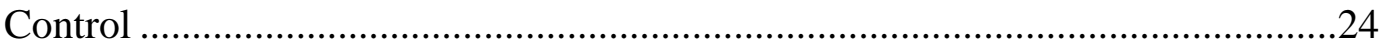

Figure 3.2 Model-based Ignition Timing Management ...................................................25

Figure 3.3 Modeled flame developed burn duration vs. experimental flame development

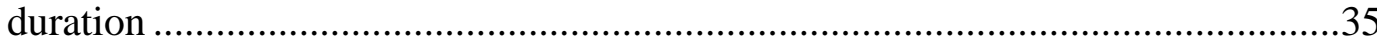

Figure 3.4 Experimental flame development period (IGN-CA05) vs. experimental rapid

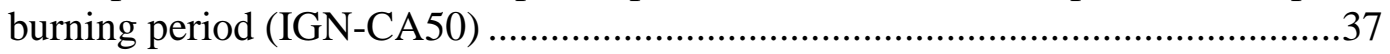

Figure 3.5 Experimental burn duration (IGN-CA50) vs. modeled burn duration (IGN-

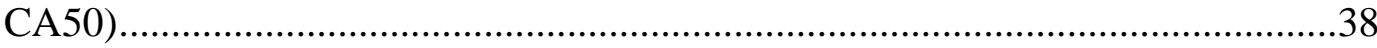

Figure 3.6 Dynamic Burn Duration and Ignition Timing Prediction ................................40

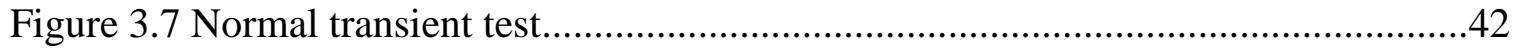

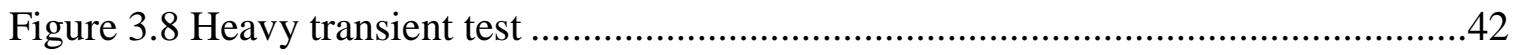

Figure 3.9 The performance of model-based burn duration and ignition prediction in a

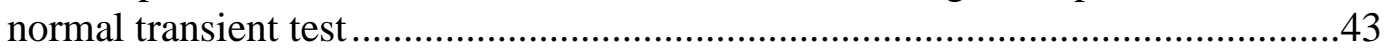

Figure 3.10 The performance of model-based burn duration and ignition prediction in a heavy transient test.............................................................................................4

Figure 3.11 Error of burn duration and ignition timing prediction....................................45

Figure 4.1 Structure of Model-Based GTDI SI Engine Control with Adaptive Ignition

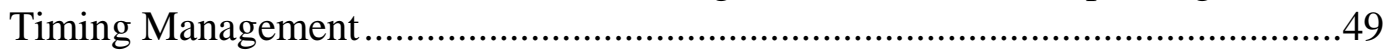

Figure 4.2 Block Diagram of Adaptive Ignition Timing Management .............................50 
Figure 4.3 Engine Operating Condition Detection .................................................54

Figure 4.4 VFF-RLS Based Combustion Model Adaptation............................................56

Figure 4.5 Transient Engine Operating Points.......................................................57

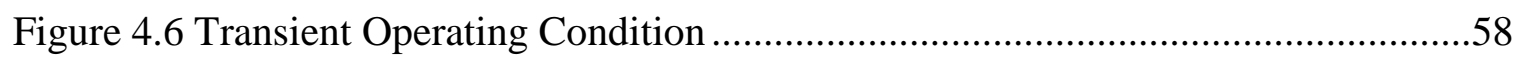

Figure 4.7 The Performance of Adaptive Burn Duration and Ignition Timing Prediction60

Figure 4.8 Errors of burn duration and ignition timing prediction ................................61

Figure 4.9 AEKF based Feedback Estimation of Burn Duration and CA50 ..................66

Figure 4.10 AEKF-Based Feedback Estimation of Burn Duration Estimation and Combustion Phasing ...........................................................................................68

Figure 5.1 Schematic of GTDI SI Engine System .............................................. 72

Figure 5.2 Model-based Cycle-by-Cycle GTDI SI Engine Control using E-NMPC ........73

Figure 5.3 GT-POWER Engine Model Development ….......................................... 76

Figure 5.4 GT-POWER Combustion Model and NOx model Calibration Results ...........78

Figure 5.5 GT-POWER Engine Model ................................................................

Figure 5.6 GT-POWER Engine Model Transient Validation.......................................80

Figure 5.7 Feedforward Neural Network Architecture ...........................................86

Figure 5.8 Feedforward Neural Network Architecture Optimization............................88

Figure 5.9 E-NMPC Engine Control Performances of the First Transient Test ..............104

Figure 5.10 E-NMPC Engine Control Actions of the First Transient Test ....................105

Figure 5.11 ISFC Results of GT-POWER DOE CAM Sweep Under Different Engine Speed and IMEPn with Fixed 8 degATDC CA50 and Wide Open Wastegate (Red stars are optimal CAM timings calculated by E-NMPC) .................................108

Figure 5.12 NOx Results of GT-POWER DOE CAM Sweep Under Different Engine Speed and IMEPn with Fixed 8 degATDC CA50 and 100\% Wastegate Opened (Red stars are optimal CAM timings calculated by E-NMPC)...........................108

Figure 5.13 ISFC and NOx Results of GT-POWER DOE CA50 Sweep with 100\% Wastegate Opened .109 
Figure 5.14 Process of Fuel Consumption and NOx Emission Comparison....

Figure 5.15 Dyno Test Engine Operating Points: Replicated from Real-Word Driving

Cycle

Figure 5.16 E-NMPC Engine Control Performances of the Real-Driving Cycle Test ....114

Figure 5.17 E-NMPC Engine Control Actions of the Real-Driving Cycle Test .............115 


\section{List of tables}

Table 2.1 Test Fuel Specifications .........................................................................21

Table 2.2 Experimental Engine Specifications ......................................................21

Table 3.1 Base Combustion Model Calibration Points....................................................34

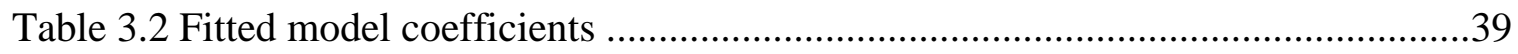

Table 3.3 Transient tests comparison.....................................................................41

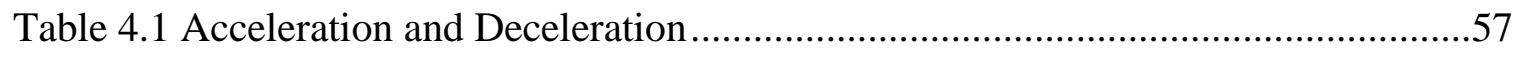

Table 5.1 Model Calibration Multipliers in GT-POWER .............................................78

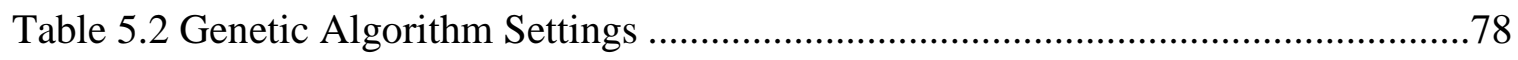

Table 5.3 GT-POWER Steady State DOE Simulation for Control-Oriented Engine Model

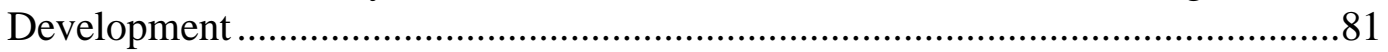

Table 5.4 Ordinates for Turbocharger Maps........................................................... 90

Table 5.5 Control-Oriented Engine Models.........................................................97

Table 5.6 Real-Word Driving Cycle Engine Acceleration/Deceleration Conditions ......113

Table 5.7 IMEPn Tracking and Control-Oriented Model Prediction Performance of ENMPC Engine Control........................................................................ 115

Table 5.8 E-NMPC Engine Control vs. Baseline Engine Control ................................116 


\section{Preface}

The goal of this research is to develop a model-based control system for cycle-by-cycle GTDI SI engine control. Chapter 3 and Chapter 4 include materials that have been published.

1. Wang, Xin, Khameneian, Amir, Dice, Paul, Chen, Bo, Shahbakhti, Mahdi, Naber, Jeffrey D., Archer, Chad, Qu, Qiuping, Glugla, Chris, and Huberts, Garlan. "ModelBased Combustion Duration and Ignition Timing Prediction for Combustion Phasing Control of a Spark-Ignition Engine Using In-Cylinder Pressure Sensors." Proceedings of the ASME 2019 International Design Engineering Technical Conferences and Computers and Information in Engineering Conference. Volume 9: 15th IEEE/ASME International Conference on Mechatronic and Embedded Systems and Applications. Anaheim, California, USA. August 18-21, 2019. V009T12A033. ASME. https://doi.org/10.1115/DETC2019-97703

This work was collaborated with Ford Motor Company to develop a model-based ignition timing management system for combustion phasing (CA50) control. This paper was peer reviewed and presented at the 2019 ASME International Design Engineering Technical Conferences and Computers and Information in Engineering Conference in Anaheim, CA, USA. The materials in this paper are included in Chapter 3.

2. Wang, Xin, Khameneian, Amir, Dice, Paul, Chen, Bo, Shahbakhti, Mahdi, Naber, Jeffrey D., Archer, Chad, Qu, Qiuping, Glugla, Chris, and Huberts, Garlan. "ControlOriented Model-Based Burn Duration and Ignition Timing Prediction with RecursiveLeast-Square Adaptation for Closed-Loop Combustion Phasing Control of a Spark Ignition Engine." Proceedings of the ASME 2019 Dynamic Systems and Control Conference. Volume 2: Modeling and Control of Engine and Aftertreatment Systems; Modeling and Control of IC Engines and Aftertreatment Systems. Park City, Utah, 
USA. October $8-11, \quad 2019 . \quad$ V002T12A004. ASME. https://doi.org/10.1115/DSCC2019-9073

This work was collaborated with Ford Motor Company to continue the development of model-based combustion phasing control. This paper was peer reviewed and presented at the 2019 ASME Dynamic Systems and Control Conference in Park City, Utah, USA. The materials in this paper are included in part of the Chapter 4.

3. Wang, Xin, Khameneian, Amir, Dice, Paul, Chen, Bo, Shahbakhti, Mahdi, Naber, Jeffrey D., Archer, Chad, Qu, Qiuping, Glugla, Chris, and Huberts, Garlan. " Cycleby-Cycle Economic Nonlinear Model Predictive Control for a Gasoline Turbocharged Direct Injection Spark-Ignited Engine."

This work was collaborated with Ford Motor Company to continue the development of model-based GTDI SI engine control. The draft will be submitted to Applied Energy. Contents are included in Chapter 5 


\section{Acknowledgements}

First of all, I would like to sincerely send my appreciation and respect to my co-advisors,

Dr. Bo Chen and Dr. Jeffrey D. Naber, who have been guiding and supporting my research throughout my Ph.D. journey. Their expertise and guidance provided great support during the difficult times in my research. Their work ethic has inspired me to be more passionate and dedicated to the field I am working in.

I also give my most gratitude and thanks to Dr. Mahdi Shahbakhti, who has offered consistent help to my research. I would also like to thank Dr. David D. Wanless for being my doctoral advisory committee and providing support.

I would like to acknowledge Ford Motor Company for their sponsorship of the research and guidance on the research. I would like to give special thanks to Garlan Huberts, Chris Glugla, Chad Archer, and Qiuping Qu from Ford Motor Company. They provided valuable suggestions and help with respect to this research.

I would like to acknowledge some of the best colleagues at APS Labs, Paul Dice, Joel Duncan, Yanyu Wang, Jiongxun Zhang, Amir Khameneian, Muralidhar Nischal, Cooper Heyne. Thanks for their help and support to all work at APS Labs. Thanks also go to my friends, Wei Luo, Ming Cheng, Chong Cao, Luting Wang, Chuanliangzi Liu, Meng Tang, Xuebin Yang, Mingyang Li, Zhihao Zhao, Zhuyong Yang, and many others.

At last, I would like to express my extreme gratitude to my parents Suqin Wang and Gongting Wang, who always support, encourage, and believe in me. 


\section{List of abbreviations}

\begin{tabular}{|c|c|}
\hline $\mathrm{AC}$ & Alternating Current \\
\hline AEKF & Adaptive Extended Kalman Filter \\
\hline ANNs & Artificial Neural Networks \\
\hline ATDC & After Top Dead Center in Compression Stroke \\
\hline BMEP & Brake Mean Effective Pressure \\
\hline $\mathrm{CA}$ & Crank Angle \\
\hline CA50 & Combustion Phasing or Location of $50 \%$ mass fraction burned \\
\hline CAFÉ & Corporate Average Fuel Economy \\
\hline COVIMEP & Coefficient of Variation of IMEP \\
\hline ECU & Engine Control Unit \\
\hline EMS & Engine Management System \\
\hline E-NMPC & Economic Nonlinear Model Predictive Control \\
\hline EVO & Exhaust Valve Opening \\
\hline FMEP & Friction Mean Effective Pressure \\
\hline GTDI & Gasoline Turbocharged Direct Injection \\
\hline $\mathrm{HEV}$ & Hybrid Electric Vehicle \\
\hline IMEPn & Net Indicated Mean Effective Pressure \\
\hline ISFC & Indicated Specific Fuel Consumption \\
\hline
\end{tabular}




\begin{tabular}{ll} 
IVC & Intake Valve Closing \\
MAP & Manifold Air Pressure \\
MFB & Mass Fraction Burned \\
MIMO & Multi-Input-Multi-Output \\
NOx & Oxides of Nitrogen \\
RGF & Residual Gas Fraction \\
RLS & Recursive Least Square \\
SI & Spark Ignition \\
SIL & Software-In-The-Loop \\
VISO & Single Input Single Output \\
SQP & Sequential Quadratic Programming \\
& \\
VFF & Three Way Catalyst Converters \\
\hline &
\end{tabular}




\section{Abstract}

To meet increasingly stringent fuel economy and emissions legislation, more advanced technologies have been added to spark-ignition (SI) engines, thus exponentially increase the complexity and calibration work of traditional map-based engine control. To achieve better engine performance without introducing significant calibration efforts and make the developed control system easily adapt to future engines upgrades and designs, this research proposes a model-based optimal control system for cycle-by-cycle Gasoline Turbocharged Direct Injection (GTDI) SI engine control, which aims to deliver the requested torque output and operate the engine to achieve the best achievable fuel economy and minimum emission under wide range of engine operating conditions.

This research develops a model-based ignition timing prediction strategy for combustion phasing (crank angle of fifty percent of the fuel burned, CA50) control. A control-oriented combustion model is developed to predict burn duration from ignition timing to CA50. Using the predicted burn duration, the ignition timing needed for the upcoming cycle to track optimal target CA50 is calculated by a dynamic ignition timing prediction algorithm.

A Recursive-Least-Square (RLS) with Variable Forgetting Factor (VFF) based adaptation algorithm is proposed to handle operating-point-dependent model errors caused by inherent errors resulting from modeling assumptions and limited calibration points, which helps to ensure the proper performance of model-based ignition timing prediction strategy throughout the entire engine lifetime. Using the adaptive combustion model, an Adaptive Extended Kalman Filter (AEKF) based CA50 observer is developed to provide filtered CA50 estimation from cyclic variations for the closed-loop combustion phasing control.

An economic nonlinear model predictive controller (E-NMPC) based GTDI SI engine control system is developed to simultaneously achieve three objectives: tracking the requested net indicated mean effective pressure (IMEPn), minimizing the SFC, and reducing NOx emissions. The developed E-NMPC engine control system can achieve the above objectives by controlling throttle position, IVC timing, CA50, exhaust valve opening (EVO) timing, and wastegate position at the same time without violating engine operating 
constraints. A control-oriented engine model is developed and integrated into the E-NMPC to predict future engine behaviors. A high-fidelity 1-D GT-POWER engine model is developed and used as the plant model to tune and validate the developed control system. The performance of the entire model-based engine control system is examined through the software-in-the-loop (SIL) simulation using on-road vehicle test data. 


\section{Introduction}

\subsection{Background and Research Motivation}

Currently, there are more than 1.2 billion vehicles on the road around the world, and about 80 million new cars are sold by the automotive industry annually. Over $59 \%$ of worldwide fossil fuel is and will be consumed by the transportation sector until the year 2050 [1]. With increasing concerns on energy security, economy, and environmental quality, the transportation industry is adopting various technologies to produce cleaner and more efficient vehicles including alternative fuels, hybrid electric vehicles (HEVs), and pure electric vehicles (EVs). However, it takes time for new technologies to penetrate the market. Figure 1.1 shows that the majority of the light-duty vehicles will still have internal combustion engines (ICEs) in the next thirty years. As a result, optimizing IC engines to improve the fuel economy and reduce emissions are valuable.
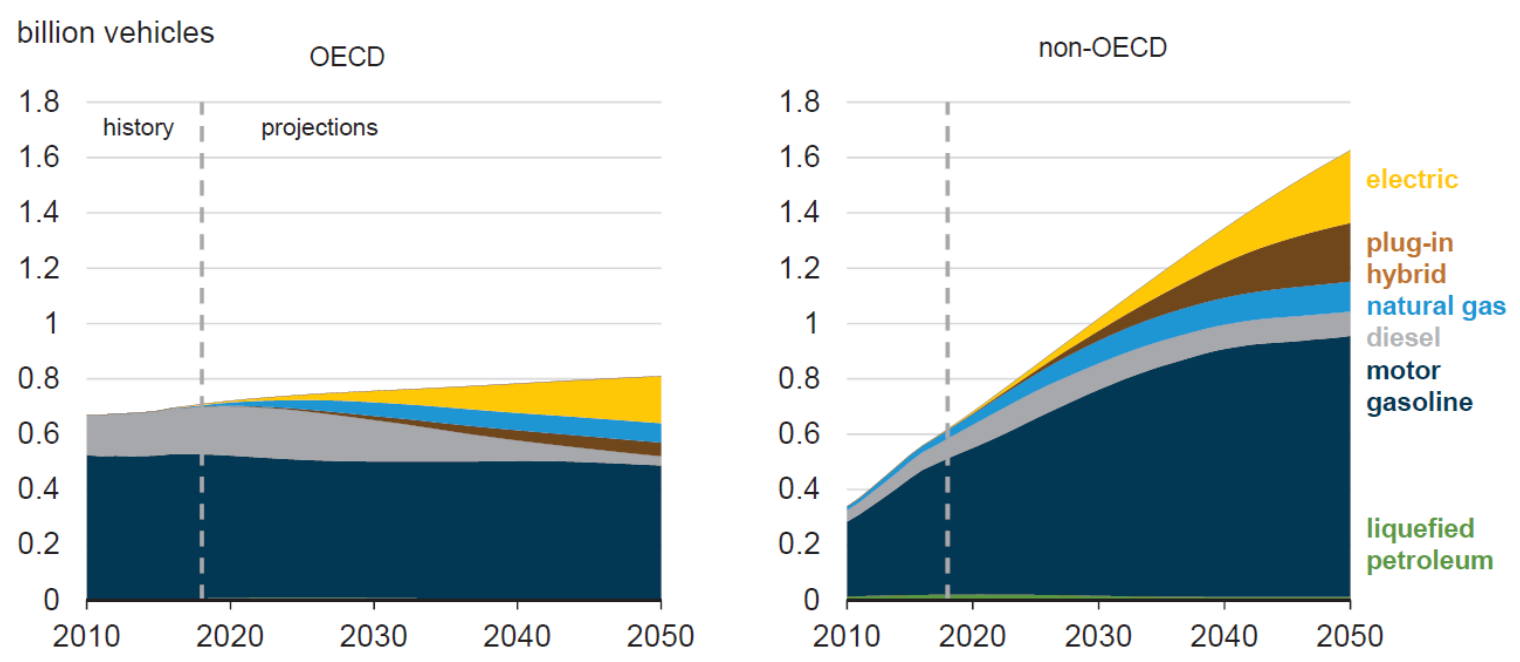

Figure 1.1 Light-duty Vehicle Stock [1] ${ }^{1}$

\footnotetext{
${ }^{1}$ OECD: Organization of Economic Cooperation and Development
} 
Spark-ignition (SI) engines fueled with gasoline are major power sources of most on-road passenger cars. The released corporate average fuel economy (CAFE) standards require light-duty vehicles to achieve 40.4 miles per gallon (mpg) fuel economy target [2], and the carbon dioxides and nitrogen oxide emissions are limited to 201 grams per mile and 0.07 grams per mile, respectively, in 2021-2026 [3]. Increasingly stringent fuel economy and emission regulations force the automotive industry to develop more efficient and cleaner SI engines by adopting various advanced technologies to production engines, including direct injection $[4,5]$, variable valve timing (VVT) [6, 7], turbocharging [8, 9], exhaust gas recirculation $[10,11]$, and variable compression ratio $[12,13]$. Among mass-production engines, GTDI SI engines equipped with VVT dominate the market due to high propulsion performance with low fuel consumption and emissions. To realize the above innovations, more advanced actuators and sensors have been equipped in modern engines, which increase the degrees of engine freedom. This results in an exponential increase of calibration work and makes conventional map-based and single-input-single-output (SISO) feedback engine control cumbersome. In addition, the traditional map-based engine control system is usually developed and calibrated for a specific engine design, and it is hard to be adapted to the future design of engine upgrading. Since the performance of GTDI SI engines, such as the response of torque delivery, fuel economy, and emission levels, are affected by the control strategies in the engine management system (EMS), developing an optimal engine control system which can deliver the requested output and operate the engine with minimum fuel consumption and emissions under wide engine operating conditions is crucial. All the above motives the researchers and the automotive industry to adopt the model-based optimal multi-input-multi-output (MIMO) control approach to develop the engine control system, which can potentially reduce the calibration work significantly, shorten the engine production cycle, and provide the capability of adapting the developed control system to similar engine designs and future engine upgrading. 


\subsection{Literature Review}

\subsubsection{GTDI SI Engine Control Problems and Approaches}

Torque control is the main control task of GTDI SI engines, which provides driver requested torque by preparing correct amount of inducted fresh air and injected fuel and igniting the in-cylinder mixtures at a proper timing. The torque-based engine control was proposed by Bosch [14] and is broadly adopted in the current production engine controllers, where the torque control module is a crucial component of an engine management system. The EMS systematically coordinates the torque control module with other control modules, including electronic throttle control, VVT management, fuel injection control, ignition timing control, turbocharger control, knock control, transmission control, tractive control, to deliver driver's torque requests. The subsystems of each control module manipulate mechanical and electrical actuators, such as electronic throttle, intake and exhaust valves, ignition coils, wastegate, to track the references generated by upper-level control modules. Currently, the rule-based torque control approach incorporating lookup tables is widely used in the production engine control unit (ECU). Extensive experimental tests are required to obtain these lookup tables. The degrees of freedom of the engine control increases with added actuators, which exponentially increases the complexity of coordinating all control modules to meet torque requirement. Many researchers have investigated various control methods for torque-based SI engine control, such as sliding-mode control [15], fuzzy gain scheduling-based PID control [16], and adaptive PID control [17]. However, the above traditional feedback control methods lack the ability to handle system constraints, require extensive calibration efforts to tune the controller parameters, and have limited working ranges.

To meet fuel economy and emission legislations, reducing fuel consumption and gaseous pollutants, such as oxides of nitrogen (NOx), carbon monoxide (CO), and hydrocarbons (HC), are two common goals of the engine control. During the warm-up period of threeway catalytic converters (TWC), engine-out emissions are high. When the TWC has been fully warmed up, over $90 \%$ of engine-out emissions can be removed, and the main task of 
EMS is to maintain stoichiometric air-fuel ratio (AFR) to achieve high conversion efficiency of TWC. Under normal engine operating conditions after the engine warms up, NOx emission rates are always high under both vehicle acceleration and deceleration due to high temperature combustion [18], and NOx emission is one of the major concerned pollutants in GTDI SI engines. In this study, we focus on reducing engine-out NOx emissions under normal engine operating conditions after the TWC is totally warmed up. Many researchers have investigated the methods of reducing NOx emission and fuel consumption through controlling VVT [19-22]. From an experimental study in [19], it shows that the objectives of minimizing the specific fuel consumption and minimizing NOx emissions conflict with each other under some engine operating conditions. The balance of these two objectives is required in the control algorithm.

For engine control, the combustion stability and engine durability are also key control requirements. Engine combustion stability is normally quantified by the coefficient of variation (COV) of IMEP, denoted as COVIMEP. High cycle-to-cycle combustion variations lead to poor drivability [23]. Engine knock causes engine damage and decreases combustion efficiency [24]. The EMS needs to operate an engine below the combustion stability threshold and engine knock limit. Furthermore, the engine controller must consider the operating limits and response delays of actuators. The calibration effort is tremendous to obtain optimal nonlinear feedforward lookup tables to simultaneously accomplish the above tasks and meet the control goals with the consideration of system constraints, and thus elongates the production cycle. 


\subsubsection{Combustion Phasing Control}

In homogeneous SI engines, the combustion of in-cylinder air-fuel mixture is initialized by the ignition spark. The mass fraction burned (MFB) curve (Figure 1.2) estimated from the measured in-cylinder pressure trace and cylinder volume data is widely used to describe the process of chemical energy release as a function of the crank angle [25]. From energy release point of view, the SI combustion process is commonly categorized into two periods, which are flame development period and rapid burning period, respectively [26]. Among the combustion matrices determined from the MFB curve, the combustion phasing (CA50) is one of the most important parameters affecting engine torque output, thermal efficiency, and emissions.

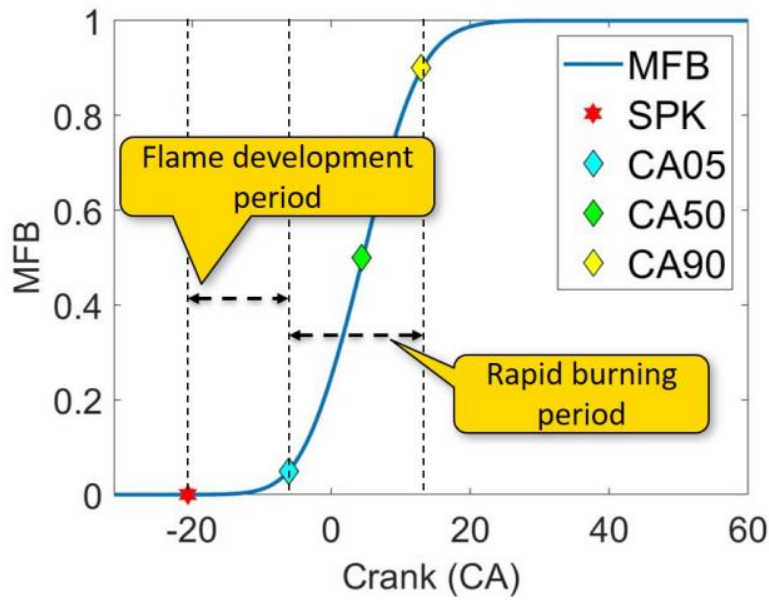

Figure 1.2 MBF of SI Combustion

For combustion phasing control in SI engines, ignition timing is used to achieve optimal CA50 targets, which are usually determined from extensive experimental tests by considering torque output response, fuel economy, emissions, and engine operating constraints. Selection of optimal CA50 values depends on the priorities of the above considerations under different engine operating conditions. Under normal engine operating conditions after the engine warms up, optimal CA50 targets are usually calibrated to achieve Maximum Brake Torque (MBT) output, which leads to the maximum engine thermal efficiency and best fuel economy [27]. Figure 1.3 shows the effect of combustion 
phasing on indicated fuel conversion efficiency [28]. Under part-load operating conditions, advanced CA50 leads to more negative work during the compression stroke and higher heat loss to the coolant, which results in lower fuel economy. Although retarding CA50 decreases peak in-cylinder temperature, which results in NOx emissions reduction [29], over retarded CA50 leads to more expansion loss and worsens the fuel economy. CA50 is correlated to two major SI engine operating constraints, which are combustion knock and combustion stability. Under high load conditions, early CA50 results in higher peak incylinder pressures and temperatures, which results in combustion knock [30] and damages the engine. Reducing emissions during engine cold start is the major task of the engine control, and the CA50 is retarded to warm up the catalyst as soon as possible [31]. However, too late CA50 may cause partial burns or misfires and leads to higher cycle to cycle combustion variations [32].

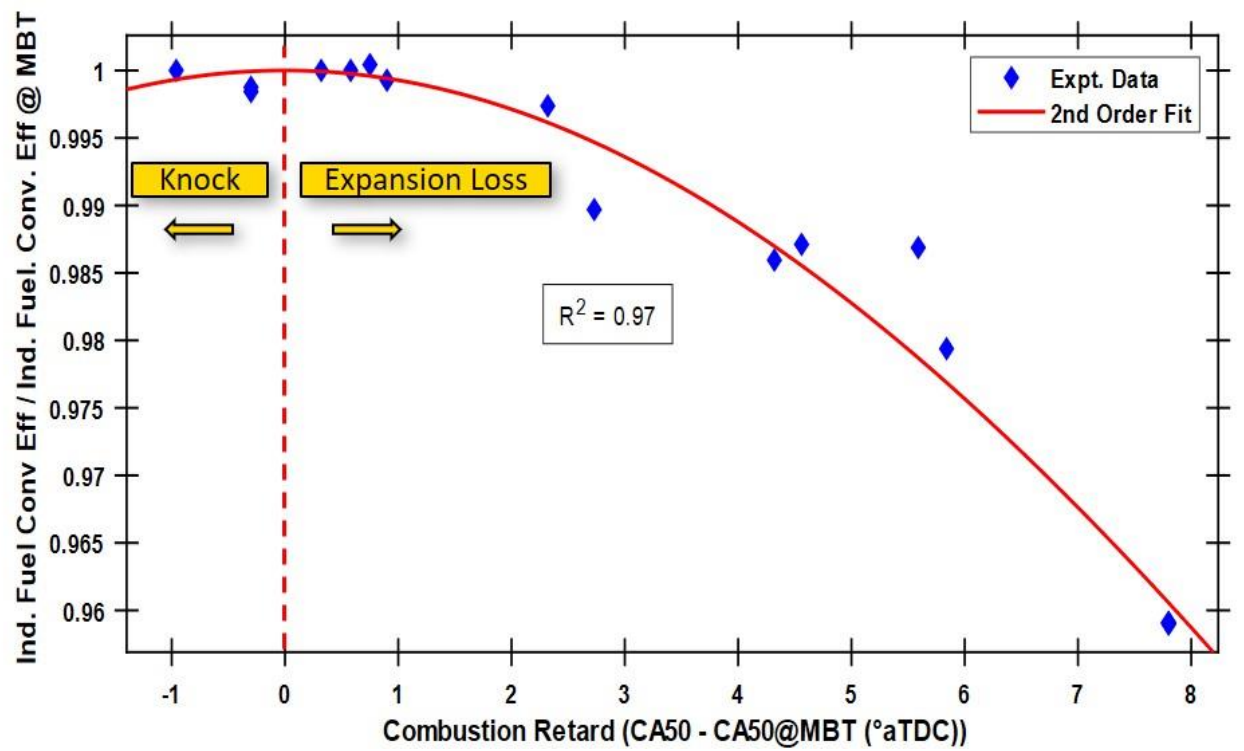

Figure 1.3 Influence of Combustion Phasing (CA50) on Engine Efficiency [28] 
Taking advantages of advanced sensors, such as the in-cylinder pressure sensor and ion sensor, traditional closed-loop feedback control, such as proportional-integral-derivative (PID) control, are widely used to manipulate the ignition timing to track target CA50 by using the estimated CA50 from measured in-cylinder pressure [33, 34] trace or ion signal $[35,36]$. In SI engines, cyclic combustion variations are natural characteristics and are normally considered as the stochastic process. The traditional feedback combustion phasing controllers calculate the ignition timing correction based on CA50 error, which is the difference between estimated CA50 of the previous cycle and CA50 target. This approach not only introduces unavoidable cycle delay, but also may lead to wrong ignition timing correction. Under transient engine operating conditions, above cycle delay and wrong correction of ignition timing can lead to severe CA50 deviation from CA50 target, which may cause combustion knock during throttle tip in maneuver and partial burn or misfire during throttle tip out maneuver. To improve the performance of the combustion phasing control under transient operating conditions, feedforward control methods are widely used in CA50 control [37]. This method helps to shorten the settling time and rising time of combustion phasing control system through outputting ignition timing needed to track the target CA50 for the upcoming cycle in the feedforward path based on current engine operating conditions. In the current production engine control units (ECUs), empirical feedforward ignition timing maps are used to achieve desired combustion phasing. These maps are obtained from lots of experimental tests. As the degree of freedom of the engine control system increases, the complexity and difficulties of deriving ignition timing control maps with considering multiple engine control objectives and constraints increase significantly. To reduce the calibration effort, both on-board calibration [38] and model-based calibration [39] methods are investigated to generate ignition timing maps from engine dyno tests and high-fidelity engine simulations. However, a large number of steady-state tests are still needed to generate optimal ignition timing maps for combustion phasing control to minimize fuel consumption and emissions. All the above motivates the automotive industry and researchers to adopt the model-based feedforward combustion phasing control. 
The process of SI combustion is complicated, which incorporates combustion chemistry, chemical kinetics, thermodynamics, fluid dynamics, and other related physics. Based on application scenarios, different kinds of SI engine combustion models have been studied and developed by researchers to be used for combustion phasing control. Depending on the level of details, the combustion models can be classified into four categories, which are zero dimensional (0-D), quasi-dimensional, one dimensional (1-D), and three dimensional (3-D) models, respectively. 0-D models are mostly developed based on the first law of thermodynamics without considering spatial resolution within the cylinder, and empirical equations are normally used to model the combustion process. In 0-D combustion models, the Vibe combustion models $[40,41]$ are widely used to model the mass fraction burn of SI combustion by tuning the model coefficients to match simulated MFB curve with the experimental MFB curve. Significant amount of calibration work is needed to ensure the accuracy of the model by creating accurate lookup tables of the model parameters. In [42, 43], the artificial neural networks (ANNs) were constructed to predict burn duration from ignition timing to CA50 ( $\Delta \theta_{\text {IGN-CA50) }}$ and CA50, respectively. However, it is not easy to adapt the trained ANN models to further engine upgrading without retraining. The quasidimensional turbulent combustion model is another type of 0-D combustion model, as the independent variable in the equations is the time or crank angle. It solves two ordinary differential equations that describe the rate of fresh fuel-air mixture entrained by the flame front and characterize the unburned turbulent eddies burning rate, respectively. The model was first proposed by Blizard and Keck [44]. Although the quasi-dimensional turbulent combustion model has the capability to predict the combustion phasing in real-time [45, 46], the model needs lots of effort to calibrate the empirical equations for laminar flame speed and turbulent intensity to fit the predicted MFB curve to the experimental MFB curve under different engine operating conditions [47]. 1-D [48, 49] and 3-D [50] combustion models have much better predictability and can provide more accurate simulation results by solving the detailed equations describing flow dynamics and chemical reactions in the cylinder. However, these models require high computing power and are not suitable for real-time engine control purpose. 
As discussed above, completely empirical combustion models have limited predictive ability and require significant calibration effort, as they lack dependencies of the physical fundamentals. The quasi-dimensional turbulent combustion models have improved predictive ability but they need to be calibrated based on engine operating conditions to guarantee the model accuracy. For the real-time combustion phasing control, computationally efficient combustion models with small amount of calibration work that can be used to calculate the needed ignition timing based on target CA50 are ideal. The parametric combustion model is a popular 0-D model which considers the physical fundamentals and has the potential to reduce the calibration work. In [51], authors derived the parametric combustion models to predict both the flame development period ( 0 to $10 \%$ MFB) and the rapid burning period ( $10 \%$ to $90 \% \mathrm{MFB}$ ). The models were developed based on the basics of turbulent flame propagation in SI engines, and only one unique set of correlations needed to be derived to provide the best model performances for a particular engine design. Different equation formats and engine operating related physical parameters, including cylinder geometry parameters, mean piston speed, laminar flame speed, turbulent flame speed, and intake mixture density, etc., have been used to develop the parametric combustion model for burn duration prediction in [52-55]. Another model of this kind uses the Arrhenius type equation to model the fuel burning rate. In [26], Heywood showed the results that Arrhenius type models coupled with rate-controlling turbulent mixing process were able to implement into multi-dimensional SI engine combustion model to simulate the MFB curve and recover in-cylinder pressure trace [56]. 


\subsubsection{Model Predictive Control and its Applications in Automotive Control Problems}

\subsubsection{Model Predictive Control}

Model predictive control (MPC), also known as the receding horizon control, is an advanced control method for solving an optimization problem. MPC is a popular and effective control method for MIMO systems, which provides a systematic control design method that naturally incorporates system constraints to achieve optimal control performance. In the MPC algorithm, dynamic system models are used to predict future system behaviors over the prediction horizon, which is defined as the time period that system models can look ahead in the future. Using predicted system states, a sequence of optimal control actions is derived for a given prediction horizon by minimizing a userdefined cost function which is subjected to a variety of system constrains, such as operating ranges of actuators, change rates of actuators, and feasible ranges of system states. The MPC is executed in every time interval and only the first control action of the derived control sequence is applied to actuators. Figure 1.4 shows an example of MIMO engine system in which E-NMPC derive a sequence of optimal control actions (U) by delivering IMEPn and minimizing $m_{c y l \_f u e l}$ and NOx while meeting system constraints over entire prediction/control horizon $(\mathrm{Nc})$. ' $\mathrm{k}$ ' is the index of the actual cycle which needs to be controlled, and ' $i$ ' is the index of cycle within the prediction horizon. The MPC algorithm can be described as follows:

1. At cycle ' $\mathrm{k}$ ', use available measurements and system models to predict system states and constraints.

2. Calculate a sequence of optimal control actions by minimizing the defined cost function over the prediction horizon while meeting system constraints using the predicted system states and constraints.

3. Implement the control actions $(U(k, 1))$ at cycle ' $k$ ' to the system.

4. Move to next cycle ' $k+1$ '. Repeat from step 1 . 

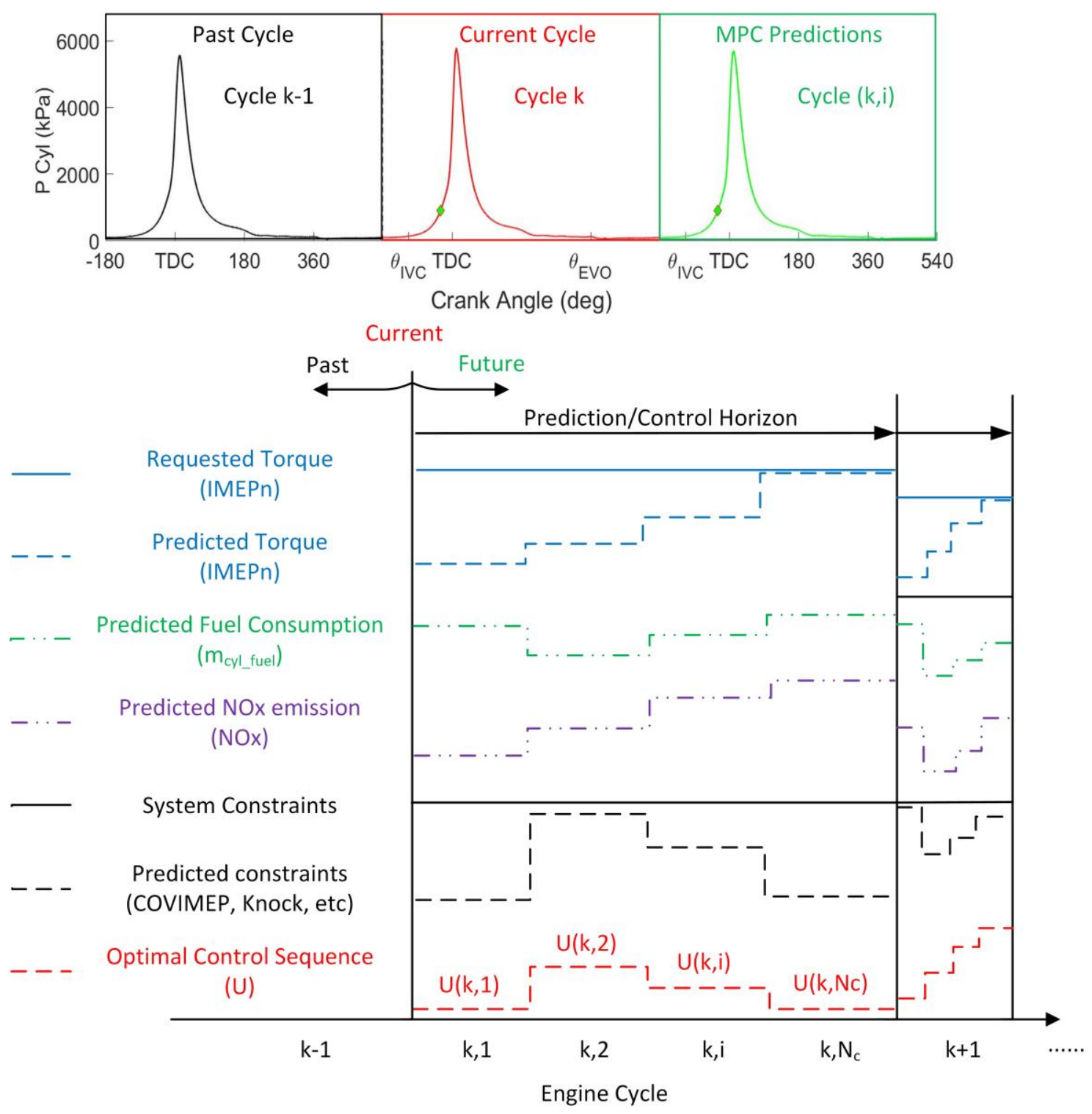

Figure 1.4 An Example of Application of E-NMPC based Engine Control

Depending on the system models and constrains, MPC can be generally classified into two categories, which is linear MPC and nonlinear MPC (NMPC), respectively. Linear MPC, uses linear system models to capture system dynamics, has been widely used in the process industries for controlling slow chemical reactions [57]. However, many systems are nonlinear. Linear models may not be able to effectively predict the future system behaviors for nonlinear systems. As the performance of the MPC heavily relies on the model 
accuracy, nonlinear models that can accurately capture the nonlinear system dynamics are needed in the MPC to predict system states over the prediction horizon. This motivates the NMPC, which uses nonlinear system models. Compared to linear MPC, NMPC needs to solve an online nonlinear constrained optimization problem, which is more computeintensive and requires powerful processors for online application. Thanks to recent progress in NMPC algorithm and advances in computing hardware, NMPC is becoming more and more popular and adopted in different industrial applications, such as agriculture [58], chemical [59], oil production [60], aircrafts [61], and automotive [62].

\subsubsection{Applications of MPC for Automotive Control}

MPC has drawn great interests from the automotive industry to push the technology for production applications due to its ability to generate optimal control actions for constrained MIMO systems to meet required control objectives, which will shorten the product development cycle and reduce calibration labor efforts. In addition, the increasing computing power of ECUs and rapid progress in solving a quadratic optimization problem online make MPC a feasible solution for vehicle control at various vehicle control levels.

In 1990s, linear MPC was first applied to IC engine for idle speed control [63]. From 2000s, MPC has been widely studied for controlling powertrain components, such as engine torque control [64-66] and transmission control [67-69]. Starting from mid-2000s, MPC has been investigated for controlling fuel cell vehicles $[70,71]$ and hybrid electric vehicles (HEVs) with different powertrain configurations, such as battery powered HEVs [72], serial HEVs [73, 74], and parallel HEVs [75, 76]. For MPC-based HEV control, energy consumption reduction [77-80] is one of the major objectives. In recent years, MPC has been investigated for future automotive applications. The stochastic MPC [81] and explicit MPC [82] has been applied for adaptive cruise control. Many researchers are applying MPC to autonomous vehicles, such as vehicle tracking control [83], optimal vehicle velocity prediction [84], steering system control [85], lane keeping [86], collision avoidance [87], and automatic emergency braking [88]. 


\subsubsection{MPC-Based Engine Control}

For engine control, MPC was initially applied for the control of diesel engine airpath in the earlier years [89-91]. Controlling a GTDI SI engine is to find sets of constrained optimal control actions to achieve multiple engine control objectives, including torque delivery, fuel consumption minimization, emission reduction, comfortable drivability, and product durability. Model-based constrained optimal MIMO control is a good option to simultaneously achieve multiple control objectives by systematically manipulating multiple actuators, and MPC is one of the candidates. One of the major advantages of MPCbased engine control is that it provides a method to naturally and explicitly incorporate engine operating constraints into an optimization problem with combined objectives, which helps to ensure the safe engine operation and significantly simplify the control algorithm development. In addition, compared to traditional control methods, the calibration of MPC is more intuitive and straightforward. Basically, there are two kinds of tunable control parameters in the MPC, which are weighting factors of each objective in a cost function and the length of prediction horizon, respectively. Based on the priorities of the engine control objectives under different engine operating conditions, the more important control objective terms will have larger weighting factors. Depending on the dynamic performance of the controlled system and control requirements, the prediction horizon should be selected in a way that stable operation of the system is ensured first, and the controller response is fast enough to satisfy the control requirements. The above calibration process of the MPC is much simpler than other traditional closed-loop controller, like PID controller, which greatly reduce the calibration time.

In recent years, MPC has been extensively investigated for controlling SI gasoline engines, and major applications of MPC on engine control are engine states/outputs tracking. To deliver the requested engine torque, MPC is used to follow the calibrated intake manifold pressure (MAP) [92, 93] and boost pressure [94, 95] targets acquired from extensive steady-state and transient engine tests by manipulating the throttle position and the wastegate position. In [65, 96], the IMEP is used as the tracking reference, and control signals of MPC are throttle air flow, EGR mass flow, and CA50. The engine shaft torque 
output is used as the control target of MPC in [64, 97, 98]. For the reduction of the fuel consumption and NOx emissions, Mingxin [99] Tae-Kyung and Lee [100] applied MPC to reduce NOx emissions and deliver the requested torque by following calibrated MAP values. Tae-Kyung Lee et al. [100] tried to achieve residual gas fraction (RGF) targets by controlling the throttle position, intake, and exhaust cam timings to follow set-points for actuators, which were calibrated at some engine operating points to minimize NOx emissions. MPC was investigated in [22] to control both intake and exhaust cam timings to track optimal trajectories of cam timings, which were calibrated to minimize fuel consumption and NOx emission.

To successfully implement MPC-based engine control, two key components are required: (1) accurate engine models (2) fast MPC execution for cycle-by-cycle engine control. Since the performance of MPC is heavily relied on the accuracy of the system models integrated in the control algorithm, developing an accurate dynamic GTDI SI engine model based on the control objectives is critical. Limited by the computing power and memory size of the engine controller, the engine model should be computationally efficient and capable of real-time evaluation. The GTDI SI engine is a complex dynamic system comprising chemical reactions, fluid mechanics, thermal dynamics, mechanical dynamics, etc. Linear engine models are widely used in the MPC-based engine control [64, 99]. However, multiple linear MPCs are required to cover wide engine operating conditions, and these linear MPCs need to be scheduled in a way to ensure the smooth and stable engine operation. As an SI engine is a nonlinear dynamic system, developing a computational efficient and accurate engine model that can effectively capture the engine dynamics and cover wide engine operating conditions is challenging. To capture the nonlinear dynamics between inputs and outputs of an engine, detailed physics-based models are needed. Incylinder combustion metrics, such as IMEPn and combustion phasing, are calculated from crank angle based in-cylinder pressure. To predict crank angle based in-cylinder pressure, detailed combustion models are needed, which are usually represented by nonlinear partial differential equations. Linearizing system and constraints through calculating sensitivity matrices is one of the key steps in the nonlinear model predictive control (NMPC) 
algorithm using the sequential quadratic programming (SQP) approach [101]. Online calculation of sensitivity matrices of system models and evaluating nonlinear system models in MPC introduce a heavy computational burden. To effectively capture system dynamics with acceptable accuracy and computation time, different kinds of modeling techniques have been investigated in MPC based engine control, such as neural network [94, 102, 103], state-space system identification [99, 104, 105], and semi-physics based control-oriented modeling [106, 107].

\subsection{Research Objectives and Tasks}

This dissertation presents a model-based control system for cycle-by-cycle control of a GTDI SI engine. The goal of the engine control system is to simultaneously track requested net indicated mean effective pressure and minimize fuel consumption and NOx emissions without violating engine operating constraints by systematically managing throttle position, intake valve closing (IVC) timing, spark timing, exhaust valve opening (EVO) timing, and wastegate opening percentage. To achieve above goal, the following tasks are required to be accomplished:

1. Develop a computationally efficient control-oriented combustion model and ignition timing prediction algorithm for cycle-by-cycle combustion phasing control.

2. Develop an adaptive algorithm for feedforward ignition timing prediction that can ensure the accuracy of model-based combustion phasing control throughout the engine lifetime and enable the combustion phasing control system to cover entire engine operating conditions without additional calibration.

3. Develop a feedback combustion phasing estimator to acquire responsive and 'filtered' CA50 estimation from cycle-by-cycle CA50 calculated using in-cylinder pressure sensor under transient engine operating conditions.

4. Develop a computationally efficient and control-oriented MIMO GTDI SI engine model that can effectively and accurately capture engine dynamics and be easily 
integrated into the model-based engine control algorithm for real-time engine control purpose.

5. Develop an economic nonlinear model predictive controller which can track requested IMEPn and minimize fuel consumption and NOx emissions with the consideration of all necessary engine operating constraints.

6. Develop a high fidelity 1D GT-POWER GTDI SI engine model to generate engine data for the development of control-oriented MIMO GTDI SI engine model. Build a virtual simulation framework to tune and evaluate the MPC controller by using the developed 1D GT-POWER virtual engine.

7. Evaluate the performance of the entire model-based engine control system, which integrates the adaptive combustion phasing controller and the E-NMPC engine controller, through the software-in-the-loop co-simulation between GT-POWER and SIMULINK using on-road vehicle test data.

\subsection{Contributions}

The major contributions of this study are summarized below:

- A model-based cycle-by-cycle feedforward ignition timing management system using a computationally efficient control-oriented combustion model has been developed. The system is evaluated using two transient tests. The developed ignition timing management algorithm can predict the needed ignition timing to achieve the target CA50 within 0.8 CAD error. With only 64 test points used for model calibration, the developed ignition timing management system is shown to cover wide engine operating conditions, which significantly reduces the calibration efforts.

- A Recursive-Least-Square (RLS) based adaptive algorithm is developed and integrated into the feedforward ignition timing management system to handle operating-point-dependent model errors, which helps to improve and maintain the accuracy of combustion model and thus ensure the performance of model-based 
combustion phasing control throughout the engine lifetime. A variable forgetting factor (VFF) is integrated into the RLS algorithm, which helps stabilize the adaptive algorithm by mitigating negative effects of stochastic cycle-to-cycle combustion variations. The developed RLS-VFF based adaptive algorithm is able to expand the original feedforward ignition timing management to cover the entire engine operating conditions without additional calibration efforts, which shows the potential of a significant reduction of calibration work.

- A CA50 observer is proposed using an adaptive Extended Kalman Filter (AEKF) and developed combustion model, which provides a method of accurate and fast estimation of CA50 from cycle-to-cycle using an in-cylinder pressure sensor under transient operating conditions. To reduce the calibration effort and the risk of divergence of the AEKF algorithm caused by improper values of covariances metrices, the recursive method is used for online estimation of covariance metrices. Compared to traditional CA50 estimation using the moving average approach, the developed AEKF-based feedback CA50 estimation can successfully 'filter' out the 'stochastic noise' and provide 'filtered' CA50 estimation without significant cycle delays, which provides an effective method for CA50 estimation under transient engine operating conditions.

- An E-NMPC based cycle-by-cycle GTDI SI engine control system is developed. The E-NMPC based engine control system aims to simultaneously accomplish three objectives: requested IMEPn delivery, fuel consumption minimization, and NOx emission reduction. A comprehensive set of engine operating constraints has been considered in the E-NMPC, including both physical limitations of actuators and constraints for safe and stable engine operation. The developed E-NMPC based engine control system can successfully achieve three control objectives by systematically manipulate actuators and meet all engine operating constraints at the same time. To the best of our knowledge, the NMPC-based cycle-by-cycle control of a GTDI SI engine to simultaneously achieve three control objectives: torque delivery, fuel consumption minimization, and NOx reduction, while considering all necessary engine constraints has not been studied yet. 
- A control-oriented MIMO GTDI SI engine model is developed by incorporating both physics-based and data-driven modeling approaches to effectively and accurately capture nonlinear engine dynamics. The developed control-oriented engine model is integrated into the E-NMPC algorithm to predict the future engine behaviors. The hybrid modeling approach helps to reduce the computational burden of E-NMPC for online engine control applications.

- A high fidelity 1D GT-POWER GTDI SI engine model is developed and calibrated using experimental data. The developed virtual engine model is validated trough transient on-road vehicle data. A software-in-the-loop co-simulation platform with GT-POWER and SIMULINK is built to tune and evaluate the model-based engine control system, which speeds up the control system development. The entire modelbased engine control system is evaluated using real world transient vehicle driving tests through SIL simulation. The SIL results demonstrate that the developed ENMPC based engine control system can track the requested IMEPn and find the best achievable combination of IVC and EVO to minimize the ISFC and reduce the NOx emission. This work provides a framework of model-based engine control, which can be easily adapted to different engine design and future upgrading by recalibrating engine models. 


\subsection{Outline of the dissertation}

According to the research objectives described above, the dissertation has been organized as following .

- Chapter 2 describes the detailed specifications of the test engine used in this study. The information of key instrumentation sensors, data acquisition system, and schematic of test engine setup are also introduced.

- Chapter 3 presents the development of a computationally efficient control-oriented combustion model for predicting burn duration (IGN-CA50). Using the developed combustion model, a framework of cycle-by-cycle feedforward model-based ignition timing management is proposed to find the ignition timing at IVC for the upcoming cycle based on the desired CA50.

- Chapter 4 expands the working ranges of the feedforward model-based ignition timing management by adopting the adaptation techniques. A Recursive-LeastSquare (RLS) with Variable Forgetting Factor (VFF) based adaptation algorithm is developed to enable the ignition timing management system to cover entire engine operating conditions and improve the combustion phasing control accuracy. The adaptive extended Kalman filter (AEKF) based CA50 estimator is proposed to provide responsive and 'filtered' feedback CA50 estimation under transient engine operating conditions.

- Chapter 5 presents an economic nonlinear model predictive controller based cycleby-cycle engine control system. The entire model-based control system aims to deliver the driver requested torque and simultaneously minimize the fuel consumption and NOx emission with the consideration of engine operating constraints. A control-oriented MIMO GTDI SI engine model, which can capture nonlinear engine dynamics and predict the future engine behavior, is integrated in the E-NMPC algorithm. A 1D engine model and a virtual testing environment is built to evaluate the performance of the E-NMPC engine control system through software-in-the-loop co-simulation between GT-POWER and SIMULINK using on-road vehicle test data. 


\section{Test Engine and Experimental Setup}

\subsection{Test Engine}

The experimental engine is a Ford 2.0L direct-injection (DI), turbocharged, 4-cylinder engine, as showed in Figure 2.1. The test engine is equipped with variable valve timing technology, and the intake cam timing and exhaust cam timing can be controlled independently. A 450kW programmable Alternating Current (AC) dynamometer was used to run the engine under desired steady-state and transient operating conditions. The test fuel is AKI 87 fuel, and fuel properties are described in Table 2.1. The detailed specifications of the engine are listed in Table 2.2.

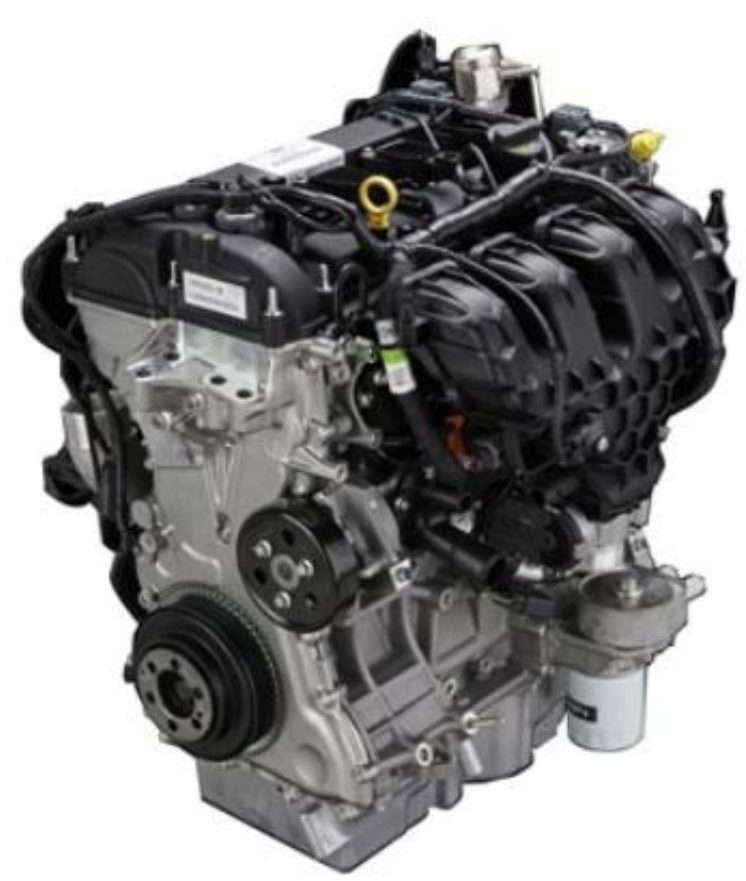

Figure 2.1 Test Engine 
Table 2.1 Test Fuel Specifications

\begin{tabular}{cc}
\hline Parameter & Value \\
\hline Carbon $(\%)$ & 83.06 \\
Hydrogen $(\%)$ & 13.48 \\
Oxygen $(\%)$ & 3.46 \\
Density $\left(\mathrm{kg} / \mathrm{m}^{3}\right)$ & 741.9 \\
Lower heating value $(\mathrm{MJ} / \mathrm{kg})$ & 41.725 \\
Stoichiometric Air-Fuel Ratio & 14.06 \\
\hline
\end{tabular}

Table 2.2 Experimental Engine Specifications

\begin{tabular}{cc}
\hline Parameter & Value \\
\hline Displacement (L) & 2.0 \\
Compression Ratio & $9.3: 1$ \\
Bore (mm) & 87.5 \\
Connecting Rode Length & 155.8 \\
$(\mathrm{~mm})$ & 83.1 \\
Stroke (mm) & $1-3-4-2$ \\
Firing Order & 247 \\
Base IVC $\left({ }^{\circ}\right.$ ATDC) & 11 \\
Base IVO $\left({ }^{\circ}\right.$ ATDC) & 8 \\
Base EVC $\left({ }^{\circ}\right.$ ATDC) & -216 \\
Base EVO $\left({ }^{\circ}\right.$ ATDC) & $270 \mathrm{lb}-\mathrm{ft} @ 3000 \mathrm{rpm}$ \\
Rated Torque & $240 \mathrm{hp} @ 5500 \mathrm{rpm}$ \\
Rated Power &
\end{tabular}




\subsection{Experimental Setup}

Figure 2.2 shows the schematic of the test engine with instrumentations. The production Bosch ECU and ATI Vision calibration software were used to control the engine and monitor all necessary engine states. The external cooling system was used to maintain the engine coolant temperature at desired values. Inlet air temperature before the throttle was controlled by the cooling temperature in the turbocharger after-cooler. The real-time combustion analysis system (CAS) from MTS powertrain technology and VeriStand system from National Instruments were used to acquire angle-based and time-based test data. Kistler 6125A piezoelectric in-cylinder pressure sensors are equipped in all cylinders to measure the crank-angle based pressure signals with a sample interval of 0.5 CAD. The measurement range and output range of in-cylinder pressure sensors are between 0 and 100 bar, and 0 to $5 \mathrm{~V}$, respectively. OMEGA and piezoresistive Kulite pressure sensors were used to measure MAP and exhaust pressure in the integrated exhaust manifold with 0.5 CAD resolution. Kulite pressure sensor is directly mounted on the exhaust manifold, to accurately capture exhaust pressure dynamics. Meriam's laminar flow measurement (LFE) was used to measure intake air mass flow through the throttle. Thermocouples were used to measure inlet air temperature and exhaust gas temperature. A volumetric flowmeter and A 80I-110S Fluke current probe were used to measure engine fuel flow and injection pulse widths. NDIR500 fast $\mathrm{CO} \& \mathrm{CO} 2$ analyzers were used to measure emissions. Figure 2.2 shows the detailed schematic of the test engine with all instrumentational sensors. 


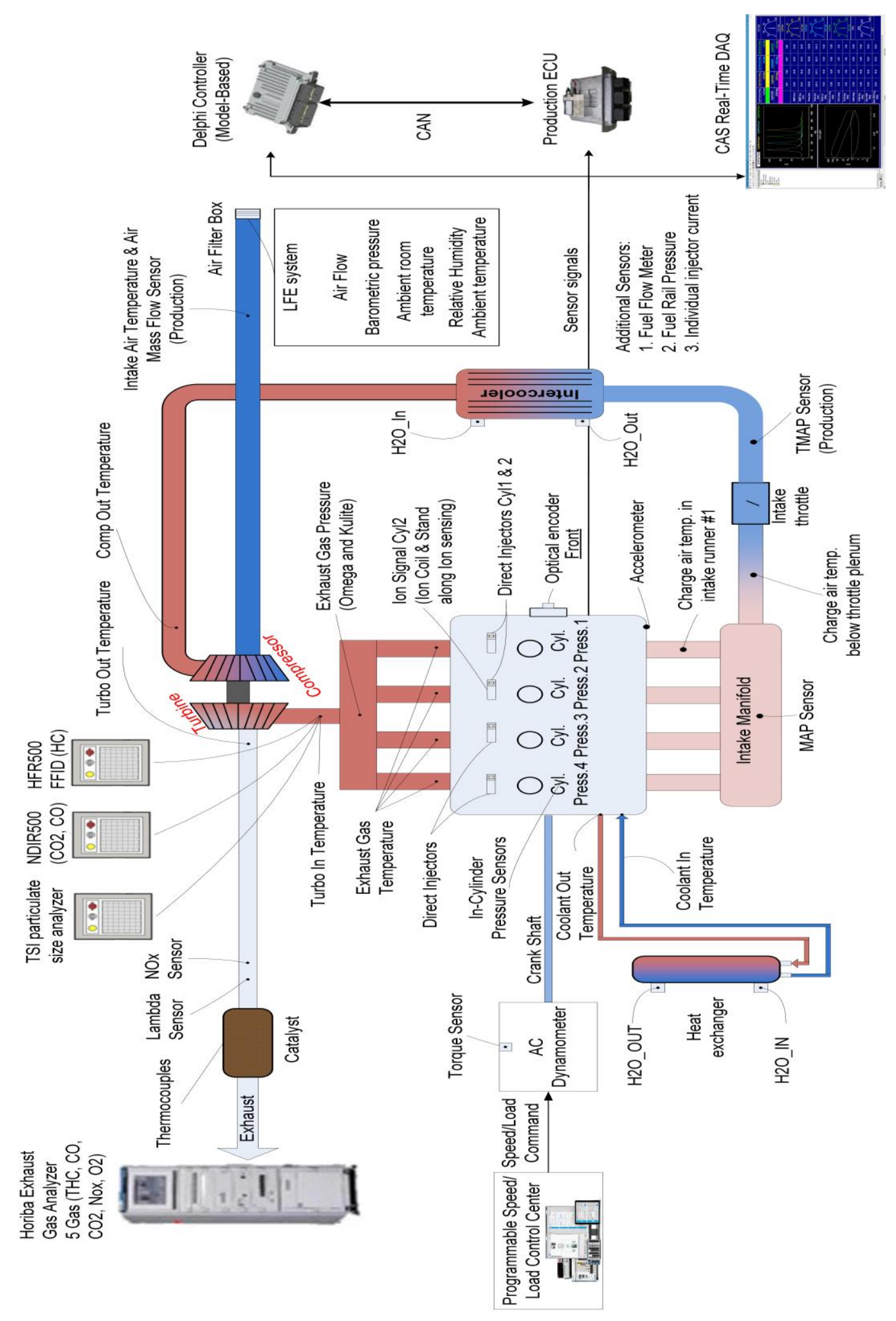

Figure 2.2 Schematic of Experimental 2.0L GTDI engine 


\section{Model-Based Combustion Duration and Ignition Timing Prediction for Combustion Phasing Control of a Spark-Ignition Engine Using In-Cylinder Pressure Sensors ${ }^{2}[108]$}

\subsection{Overview of Model-Based Burn Duration and Ignition Timing Management for Combustion Phasing Control}

The architecture of the model-based GTDI SI engine control is shown in Figure 3.1. This chapter focuses on the Model-Based Ignition Timing Management block. The entire engine cycle is defined from IVC(k-1) to IVC(k). Cycle ' $\mathrm{k}$ ' is the upcoming cycle that needs the predicted ignition timing for combustion phasing control and requires the estimation with enough time for scheduling the ignition dwell. In this work, the estimation is produced at IVC for the cycle under consideration. $\theta_{I G N}(k)$ is the final predicted ignition timing. An optimal feedforward ignition timing is determined by engine operating conditions and the desired combustion phasing, $\theta_{\text {CA50_Target }}(k)$.

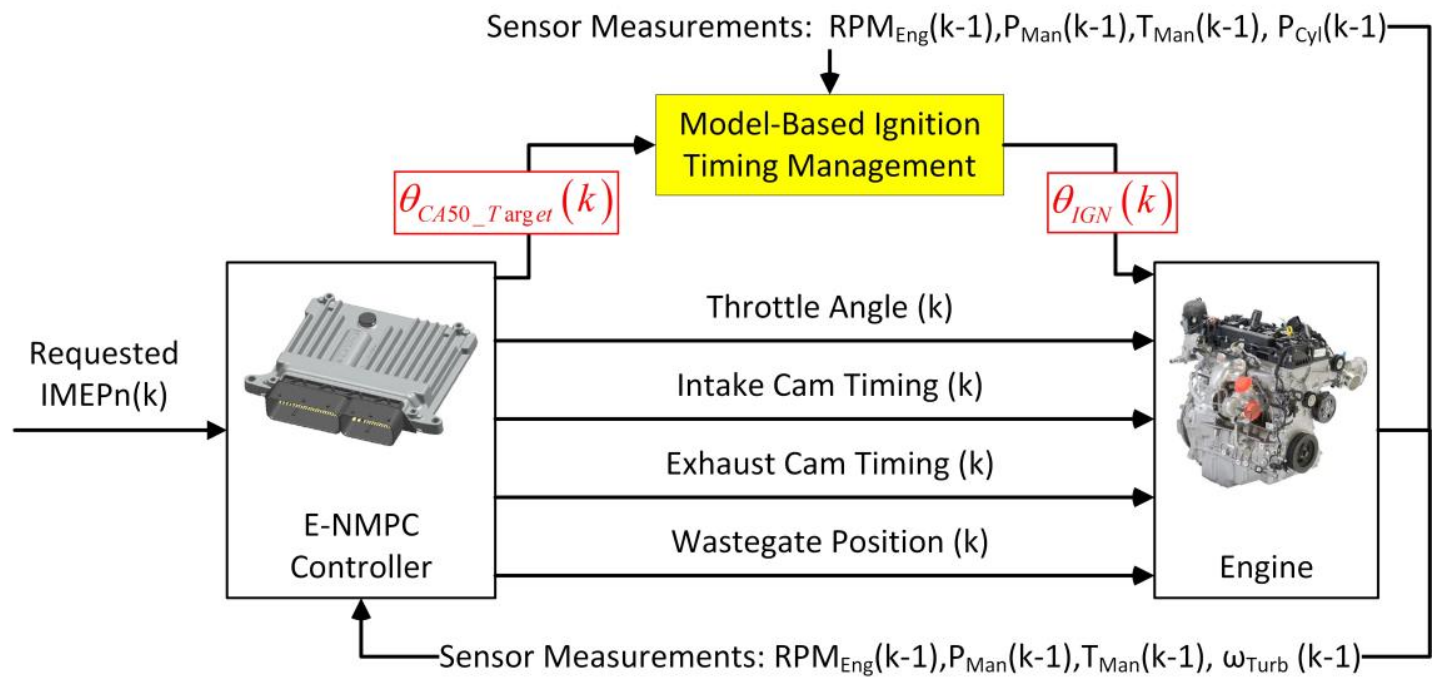

Figure 3.1 Structure of Model-Based Combustion Phasing Control in GTDI SI Engine Control

\footnotetext{
${ }^{2}$ The material contained in this chapter was previously published in the Proceedings of ASME 2019 International Design Engineering Technical Conferences \& Computers and Information in Engineering Conference, Volume 9, 2019 (See Appendix 1)
} 
Figure 3.2 shows a diagram of model-based ignition timing prediction. The controloriented combustion model uses only the information at and before IVC. $\Delta \widehat{\theta}_{I G N-C A 50}^{-}(k)$ is the predicted burn duration using the developed combustion model and a dynamic burn duration and ignition timing prediction algorithm is developed to obtain the final predicted ignition timing $\theta_{I G N}(k)$, for upcoming cycle ' $\mathrm{k}$ '. A dynamic model is used to provide the needed inputs of the combustion model, including in-cylinder temperature and the masses of both inducted fresh air and residual gases at IVC cycle-by-cycle [109].
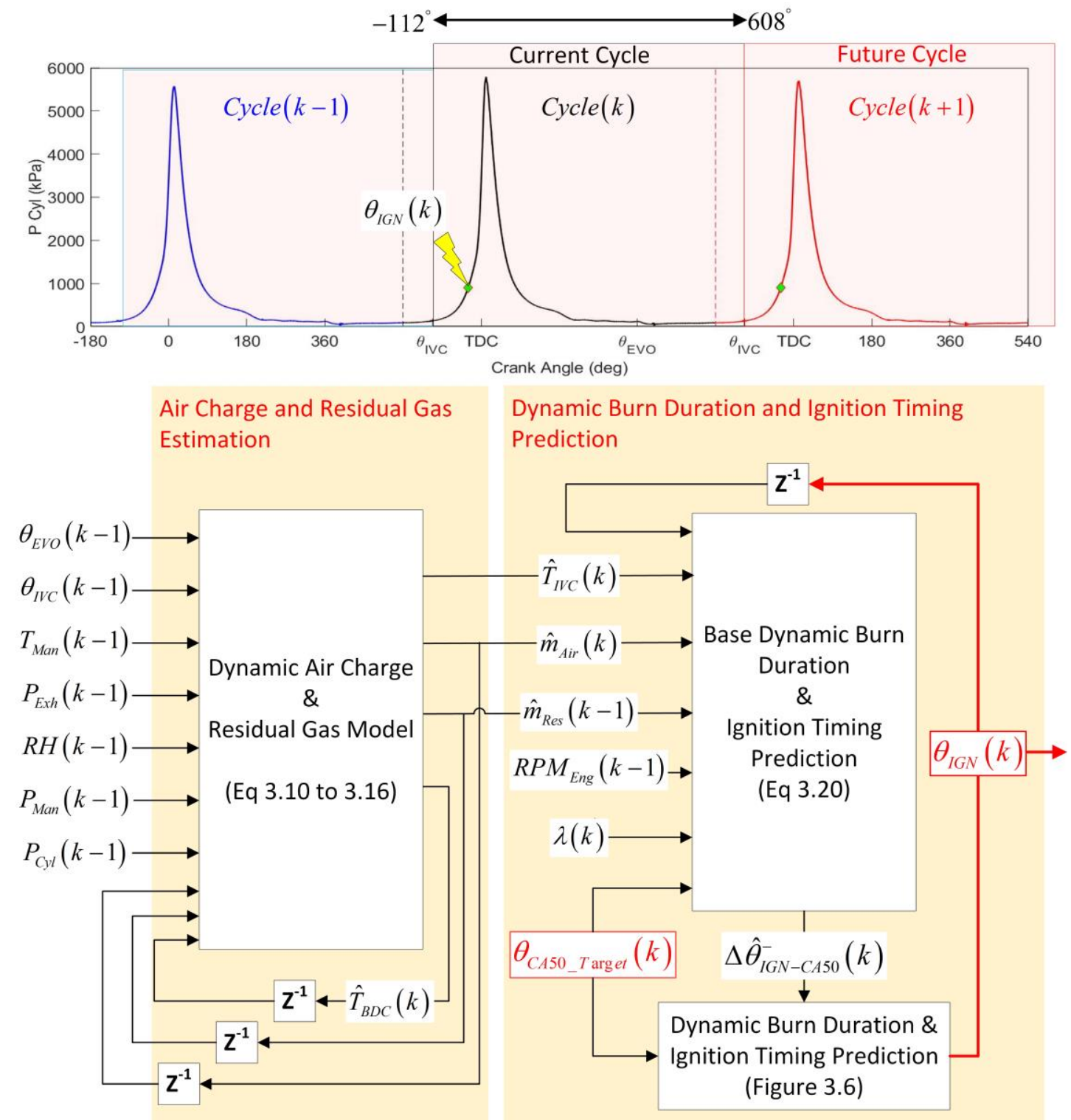

Figure 3.2 Model-based Ignition Timing Management 


\subsection{SI Combustion Modeling for Engine Control}

Combustion phasing is one of the most important parameters affecting engine efficiency, torque output, and emissions. In homogeneous spark-ignition (SI) engines, ignition timing control algorithms are typically map-based with several multipliers, which require significant calibration efforts. This chapter presents a framework of model-based ignition timing prediction using a computationally efficient control-oriented combustion model for the purpose of real-time combustion phasing control. Burn duration from ignition timing to CA50 ( $\left.\Delta \theta_{\text {IGN-CA50 }}\right)$ on an individual cylinder cycle-by-cycle basis is predicted by the combustion model developed in this work. The model is based on the physics of turbulent flame propagation in SI engines. The most important control parameters, including ignition timing, variable valve timing, air-fuel ratio, and the engine load mostly affected by the combination of the throttle opening position are included in the combustion model. With 64 test points used for model calibration, the developed combustion model is shown to cover wide engine operating conditions, thereby significantly reducing the calibration effort. A Root Mean Square Error (RMSE) of 1.7 Crank Angle Degrees (CAD) and correlation coefficient $\left(\mathrm{R}^{2}\right)$ of 0.95 illustrate the accuracy of the calibrated model. On-road vehicle testing data are used to evaluate the performance of the developed model-based burn duration and ignition timing algorithm. When comparing the model predicted burn duration and ignition timing with experimental data, $83 \%$ of the prediction error falls within \pm 3 CAD.

Modeling the SI combustion process is a challenging task, due to the complex combustion chemistry coupled with turbulent flow. Detailed physics-based models need to incorporate combustion chemistry, chemical kinetics, thermodynamics, fluid dynamics, and other related physics. Various kinds of SI engine combustion models, from 0-D to 3-D models, have been developed and studied to obtain the ignition timing for combustion phasing control. 0-D models employ the empirical equations and incorporate the first law of thermodynamics without accounting for spatial resolution within the cylinder [110]. 3-D models are capable of providing the most accurate simulation results by solving the equations describing flow and interaction of the fluid within the cylinder, however 3-D 
models require high computing power. Real-time combustion phasing control using 0-D combustion models is feasible to be implemented in current production ECUs. Due to lacking dependencies of physical fundamentals, completely empirical 0-D models, such as the Wiebe function, have limited predictive ability [111]. Physics-based combustion models have improved predictive ability, such as the quasi-dimensional turbulent combustion model [44, 112]. It derives the MFB curve by solving two differential equations, which phenomenologically characterize the burning rate of turbulent eddies and the entrainment rate of unburned air-fuel mixture. To attain high model accuracy, lots of engine calibration data is still required to generate lookup tables of calibration parameters, which are normally a function of engine operating conditions.

Taking into consideration the computing power of ECUs, predictive ability, and calibration efforts, this research develops a computationally efficient control-oriented combustion model to predict the burn duration $\left(\Delta \theta_{\text {IGN-CA50 }}\right)$ cycle-by-cycle. The model is developed upon the basis of turbulent flame propagation in SI engines, which considers physical fundamentals. Based on target CA50, ignition timing can be calculated from predicted burn duration $\left(\Delta \theta_{\text {IGN-CA50) }}\right.$ cycle-by-cycle using a rule-based method developed in this work. The combustion model uses the information acquired at or before the Intake Valve Closing (IVC), the starting point of the upcoming engine cycle. Since on road SI engines are often operating in transient conditions, this model-based in-cycle ignition timing prediction has significant potential to improve fuel economy and reduce emissions by maintaining the optimal combustion phasing.

\subsection{Control-Oriented Combustion Model Development}

From the heat release point of view, the combustion process of SI engines can be separated into two stages [26]. First period is the time interval between the ignition timing and the start of the combustion (SOC), which is named as early flame development stage. SOC here is defined to be the crank angle location of 5 percent mass fraction burn (CA05). The 
second period is from the CA05 to the time when the bulk of fuel-air mixture charge is burned, and this part is defined as the rapid burning stage. Since the objective is to predict the ignition timing for the coming cycle based on desired CA50, a combustion duration model that can predict burn duration between the point of start of ignition and CA50 cycleby-cycle is needed. In this study, the burn duration prediction, $\Delta \widehat{\theta}_{I G N-C A 50}^{-}$, is composed of

flame development stage, $\Delta \widehat{\theta}_{I G N-C A 05}^{-}$, and rapid burning stage, $\Delta \widehat{\theta}_{C A 05-C A 50}^{-}$, as described in Equation (3.1),

$$
\Delta \hat{\theta}_{I G N-C A 50}^{-}=\Delta \hat{\theta}_{I G N-C A 05}^{-}+\Delta \hat{\theta}_{C A 05-C A 50}^{-}
$$

\subsubsection{Flame Development Stage Model}

Based on the basics of turbulent flame propagation in SI engines, Hirs et al. developed two models for both the flame development stage and the rapid burning stage [51], as described in Equation (3.2). In Equation (3.2), the first term models the burn duration of early flame development stage, and the second term describes the burn duration of rapid burn stage. In [51], crank angle based quasi-dimensional turbulent combustion model was integrated from ignition timing to the End of the Combustion (EOC), where EOC was defined to be the crank angle of 99 percent mass fraction burned. Two assumptions were made to derive the model for flame development period: (i) the time duration between ignition timing and CA05 is proportional to the time needed to burn a turbulent eddy and (ii) turbulent intensity is positively correlated with mean piston speed. For the derivation of the combustion duration model of the rapid burning stage, 'rapid distortion' theory was used to calculate the turbulent intensity. 


$$
\begin{aligned}
& \Delta \hat{\theta}_{I G N-E O C}^{-}=\Delta \hat{\theta}_{I G N-C A 05}^{-}+\Delta \hat{\theta}_{C A 05-E O C}^{-}= \\
& a_{1}^{\prime} \cdot\left(\overline{S_{P}} \cdot v_{u n_{-} C A 05}\right)^{\frac{1}{3}} \cdot\left(\frac{h_{C A 05}}{S_{L_{-} C A 05}}\right)^{\frac{2}{3}}+a_{2} \cdot\left(\frac{B_{\text {bore }}}{h_{C A 50}}\right)^{\frac{1}{3}} \cdot\left(\frac{\rho_{\text {un_CA05 }}}{\rho_{\text {un_ } C A 50}}\right)^{\frac{10}{9}}
\end{aligned}
$$

where, $a_{1}^{\prime}$ and $a_{2}^{\prime}$ are calibration parameters. The subscripts of the parameters refer to quantities calculated at certain crank angle locations (CA05, CA50, CA90). $\overline{S_{P}}$ is the mean piston speed. $v_{\text {un_CA50 }}$ is the kinetic viscosity of unburned mixture at CA05. $h_{C A 50}$ is the effective chamber height at CA05. $S_{L_{-} C A 05}$ is the laminar flame speed calculated at CA05.

In this work, the control-oriented combustion model for the burn duration prediction of $\Delta \widehat{\theta}_{I G N-C A 05}^{-}$is derived based on the first term in (3.2). Following the turbulent combustion model developed in [51], this work also uses the two zone combustion concept, which assumes that flame front moves at turbulent flame speed and the cylinder is separated into the burned zone and unburned zones. The thermodynamic properties of the mixture in the unburned zone are calculated by assuming polytropic process. Kinetic viscosity, $v_{\text {un_CA50 }}$, is calculated using Equations (3.3) to (3.5). Equation (3.4) describes the temperature dependent dynamic viscosity correlation developed in [26]. The ranges of temperature, pressure, and equivalence ratio of mixture covered by this correlation are 500 to $4000 \mathrm{~K}, 1$ to 100 bar, and $\varphi$ is from 1 to 4 . Equation (3.6) gives calculation of mean piston speed from engine speed (rpm). 


$$
\begin{gathered}
v_{u n_{-} C A 05}=\frac{\mu_{u n_{-} C A 05}}{\rho_{u n_{-} C A 05}} \\
\mu_{u n_{-} C A 05}=3.3 \cdot 10^{-7} \cdot T_{u n_{-} C A 05}^{0.7} \\
\rho_{u n_{-} C A 05}=\frac{P_{u n_{-} C A 05}}{R \cdot T_{u n_{-} C A 05}} \\
\overline{S_{P}}=\frac{2 \cdot l \cdot R P M_{E n g}}{60} \\
h_{C A 05}=\frac{V_{C A 05}}{\pi \cdot\left(\frac{B}{2}\right)^{2}}
\end{gathered}
$$

Where, $\mathrm{RPM}_{\mathrm{Eng}}$ is the engine speed. B is the diameter of piston. 1 is the length of connecting rod. $\mathrm{V}_{\mathrm{CA} 05}$ is the cylinder volume at CA05.

By substituting Equations (3.3) to (3.6) into Equation (3.2) and combining all the constants of physical parameters into $\mathrm{a}_{1}$ and $\mathrm{a}_{2}$, predicted burn duration from start of ignition timing to EOC can be described in Equation (3.8). $\Delta \hat{\theta}_{I G N-E O C}^{-}$is a function of engine specifications, effective chamber height, temperatures and pressures of unburned zone at CA05 and CA50, laminar flame speeds at CA05 and CA50, and engine speed.

$$
=\left(\begin{array}{l}
\Delta \hat{\theta}_{I G N-E O C}^{-}=\Delta \hat{\theta}_{I G N-C A 05}^{-}+\Delta \hat{\theta}_{C A 05-E O C}^{-} \\
a_{1}^{\prime} \cdot\left(\frac{R \cdot T_{u n_{-} C A 05}}{P_{C A 05}}\right)^{\frac{1}{3}} \cdot\left(\frac{h_{C A 05}}{S_{L_{-} C A 05}}\right)^{\frac{2}{3}} \\
+a_{2}^{\prime} \cdot \frac{B_{b o r e}}{h_{C A 50}} \cdot\left(\frac{R \cdot T_{\text {un_CA50 }}}{P_{C A 50}}\right)^{\frac{1}{3}} \cdot\left(\frac{h_{C A 50}}{S_{L_{-} C A 50}}\right)^{\frac{2}{3}}
\end{array}\right) \cdot R P M_{E n g}
$$


In this work, it is assumed that the unburned mixture is not significantly compressed between ignition timing and SOC. To simplify the model without missing the physical parameters related to early flame development stage, $T_{I G N}$ and $P_{I G N}$ will be used in the first term of Equation (3.8). From parametric studies by Hirs et al. [51], the turbulent flame speed has a weak dependence on turbulent length scale, which is proportional to effective chamber height. Since CA05 is needed for calculating $h_{C A 05}$, to further simplify the model and calculation without predicting CA05, $h_{C A 05}$ in Equation (3.8) is substituted by $h_{C A 05}$. The laminar flame speed correlation developed in [113] is used in this study, as described in Equation (3.9). $Y_{F}$ and $Y_{O 2}$ represent the mole fractions of fuel and oxygen in the unburned mixture, which describe the contents of air fuel mixture trapped in the cylinder including fresh air, residuals, fuel, etc. The laminar flame speed is dependent upon the total dilution from excess air and residuals [26]. To account for this, a dilution factor $X_{\text {Dilution }}$ is defined and used to substitute $Y_{F} \cdot Y_{O 2}$, as shown in Equation (3.10). The laminar flame speed is more sensitive to residual gas fraction than to excess air dilution [26]. Since the effect of residuals and air are not the same, a parameter ' $d$ ' is introduced.

$$
\begin{aligned}
& S_{L_{-} C A 05}=\left(\frac{2 \sqrt{2} \cdot T_{u n_{-} C A 05} \cdot P_{u n_{-} C A 05}^{-0.22}}{\sqrt{51} \cdot\left(T_{m i x_{-} C A 05}\right)^{-0.5}}\right) \cdot\left(Y_{F}^{-0.71} \cdot Y_{O_{2}}^{-0.71}\right)^{0.5} \cdot\left[\mathrm{e}^{\frac{-36.8}{R \cdot T_{u n_{-} C A 05}}}\right] \\
& X_{\text {dilution }}=\frac{m_{\text {air_IVC }}+m_{\text {fuel_IVC }}}{m_{\text {air_IVC }}+m_{\text {fuel_IVC }}}+R G F+d \cdot \text { Dilution }_{\text {Air }} \\
& =1+R G F+\bar{d} \cdot \text { Dilution }_{\text {Air }}
\end{aligned}
$$

Where, $m_{\text {air_IVC }}$ is the mass of inducted fresh air at IVC. $m_{\text {fuel_IVC }}$ is the mass of injected fuel into the cylinder. Dilution Air $_{\text {is }}$ the term used to represent the excess air dilution.

The burned gas that remains in the cylinder from previous cycle reduces the adiabatic flame temperature, and hence reduces the laminar flame speed, thus the higher RGF results in longer burn duration. The mass of residuals remained in the cylinder is mainly affected by 
the intake and exhaust valve timing events especially the pressure ratio of exhaust to intake, exhaust to in-cylinder, and intake to in-cylinder [114]. Residuals also increase as engine speed decreases as there is more time for exhaust gases to flow back through the intake [115]. The model developed in $[109,116]$ is used to estimate the mass of induced fresh air and residual gas trapped in the cylinder at IVC cycle-by-cycle. RGF is defined in Equation (3.11). Equation (3.12) describes the total mass of residual trapped in the cylinder at IVC, which considers both the backflow of exhaust during the valve overlap, $\widehat{m}_{\text {exh_backflow }}$, and trapped burned gas at Intake Valve Opening (IVO), $\widehat{m}_{\text {res_trapped_IVo }}$. The mass of trapped residual gases at IVO is computed using the ideal gas law, as shown in Equation (3.13).

$$
\begin{gathered}
R G F=\frac{\hat{m}_{\text {residual_IVC }}}{\hat{m}_{\text {residual_IVC }}+\hat{m}_{\text {air_IVC }}} \\
\hat{m}_{\text {residual_IVC }}=\hat{m}_{\text {res_trapped_IVO }}+\hat{m}_{\text {exh_backflow }} \\
\hat{m}_{\text {res_trapped_IVO }}=\frac{P_{\text {IVO }} \cdot V_{\text {IVO }}}{R_{\text {residual }} \cdot \hat{T}_{\text {IVO }}}
\end{gathered}
$$

Crank angle based in-cylinder pressure is compared to the exhaust pressure. To calculate the mass of exhaust flows back into the cylinder during the valve overlap, the compressible ideal gas flow correlation described in Equation (3.14) is used when pressure ratio of downstream to upstream is larger than critical pressure ratio. The choked flow correlation described in Equation (3.15) is used when the pressure ratio of downstream to upstream is less or equal to the critical ratio. Upstream is the in-cylinder pressure, and downstream pressure is the exhaust manifold pressure. 


$$
\begin{aligned}
& \hat{m}_{\text {exh_backflow }}=\frac{\left(\frac{2 \gamma}{\gamma-1}\right)^{\frac{1}{2}}}{12 \cdot R P M_{\text {Eng }} \cdot \sqrt{R_{\text {residual }} \cdot T_{u p}}} \\
& \cdot \int_{I V O}^{E V C} C_{D} A \cdot P_{u p} \cdot\left(\frac{P_{d o w n}}{P_{u p}}\right)^{\frac{1}{\gamma}} \cdot\left\{\left[1-\left(\frac{P_{d o w n}}{P_{u p}}\right)^{\frac{\gamma-1}{\gamma}}\right]\right\}^{\frac{1}{2}} \cdot d t \\
& \hat{m}_{\text {exh_backflow }}=\frac{(\gamma)^{\frac{1}{2}}}{12 \cdot R P M_{\text {Eng }} \cdot \sqrt{R_{\text {residual }} \cdot T_{u p}}} \cdot\left(\frac{2}{\gamma+1}\right)^{\frac{\gamma+1}{2(\gamma-1)}} \cdot \int_{I V O}^{E V C} C_{D} A \cdot P_{u p} \cdot d t
\end{aligned}
$$

Where, $C_{D} A$ is the discharge coefficient multiplied by the effective port area calculated using a flow test bench. Superscripts 'up' and 'down' indicate the pressure and temperature at upstream and downstream respectively.

Equation (3.16) defines the excess air dilution term described as the percentage of excess air in the mixture. Excess air inducted into the cylinder makes the engine operate under lean condition. Mixture burning speed reaches its maximum at slightly rich conditions and decreases as the mixture becomes leaner or richer [26]. Burn duration increases as burning velocity decreases.

$$
\text { Dilution }_{\text {Air }}=\frac{\hat{m}_{\text {air_IVC }}-\hat{m}_{\text {air_stoi }}}{\hat{m}_{\text {air_IVC }}+\hat{m}_{\text {fuel_lVC }}}=\frac{\lambda-1}{\lambda+F A R_{\text {Stoi }}}
$$

Where, $\lambda$ is defined as the value of actual air to fuel ratio divided by stoichiometric air to fuel ratio. FAR Stoi $_{\text {is }}$ the fuel to air ratio at stoichiometric condition.

By substituting Equations (3.9) and (3.10) into the first term of Equation (3.8) and merging all the model coefficients, the newly developed control-oriented burn duration model for early flame development stage can be described in Equation (3.17). Due to the physics of the flame development and propagation in SI engines after the ignition timing, burn 
duration of flame development stage has the minimum limit, which is a positive value. Based on this, $a_{7}$ is added to be the physical lower constraint for early flame development stage.

$$
\Delta \hat{\theta}_{I G N-C A 05}^{-}=a_{1} \cdot \frac{\left(P_{I G N}\right)^{a_{2}}}{\left(T_{I G N}\right)^{a_{3}}} \cdot \mathrm{e}^{\left(\frac{a_{4}}{T_{I G N}}\right)} \cdot\left(1+R G F+a_{5} \cdot \frac{\lambda-1}{\lambda+F A R_{\text {stoi }}}\right)^{a_{6}} \cdot R P M+a_{7}
$$

Where, $a_{1}$ to $a_{7}$ are model coefficients that need calibration.

To obtain the coefficients of Equation (3.17), an optimization method is used to minimize the RMSE between predicted burn duration, $\Delta \hat{\theta}_{I G N-C A 05}^{-}$, and experimental burn duration, $\Delta \theta_{I G N_{-} C A 05}$. MATLAB function 'fmincon' was used to determine values of $a_{1}$ to $a_{7}$ to minimize Equation (3.18) using the 'Global Search' method. In 'fmincon', the boundaries of coefficients were chosen to ensure that changes of physical model parameters resulting in correct directional changes of modeled burn duration. The combustion model development data set listed in Table 3.1, composed of 64 test points, was used to derive model coefficients.

$$
R M S E=\sqrt{\frac{\sum_{m=1}^{n}\left(\Delta \hat{\theta}_{I G N-C A 05}^{-}(m)-\Delta \theta_{I G N-C A 05}(m)\right)^{2}}{n-1}}
$$

Where, $\mathrm{n}$ is the number of experimental data points.

Table 3.1 Base Combustion Model Calibration Points

\begin{tabular}{|c|c|c|c|c|c|}
\hline $\begin{array}{c}\text { Total } \\
\text { Points }\end{array}$ & $\begin{array}{c}\text { Engine } \\
\text { Speed } \\
(\text { RPM) }\end{array}$ & $\begin{array}{c}\text { Gross } \\
\text { IMEP } \\
(\mathbf{k P a})\end{array}$ & $\begin{array}{c}\text { CA50 } \\
\left({ }^{\circ} \text { ATDC) }\right.\end{array}$ & $\begin{array}{c}\boldsymbol{\lambda} \\
(-)\end{array}$ & $\begin{array}{c}\text { Cam } \\
\text { Overlap } \\
\text { (CA deg) }\end{array}$ \\
\hline 64 & $\begin{array}{c}1500 \& \\
6500\end{array}$ & $250 \& 750$ & $8 \& 18$ & $0.9 \& 1$ & $0 \sim 47$ \\
\hline
\end{tabular}


Figure 3.3 shows the results of the flame development model fit. The $\mathrm{x}$-axis represents the experimental results of IGN-CA05 burn duration, and the y-axis represents the modeled IGN-CA05 with the fitted coefficients. The points in the figure are color coded according to the residual gas fraction as shown in the color bar to the right of the figure. It can be observed that the duration of the flame development stage increases with increasing RGF. This is because at higher RGF the laminar burning velocity decreases. The RMSE of $\Delta \hat{\theta}_{I G N-C A 05}^{-}$is $1.7 \mathrm{CAD}$, and the correlation coefficient is 0.95 . The red dashed lines indicate $\pm 10 \%$ error, and $85 \%$ of predicted burn durations are within $\pm 10 \%$ error lines.

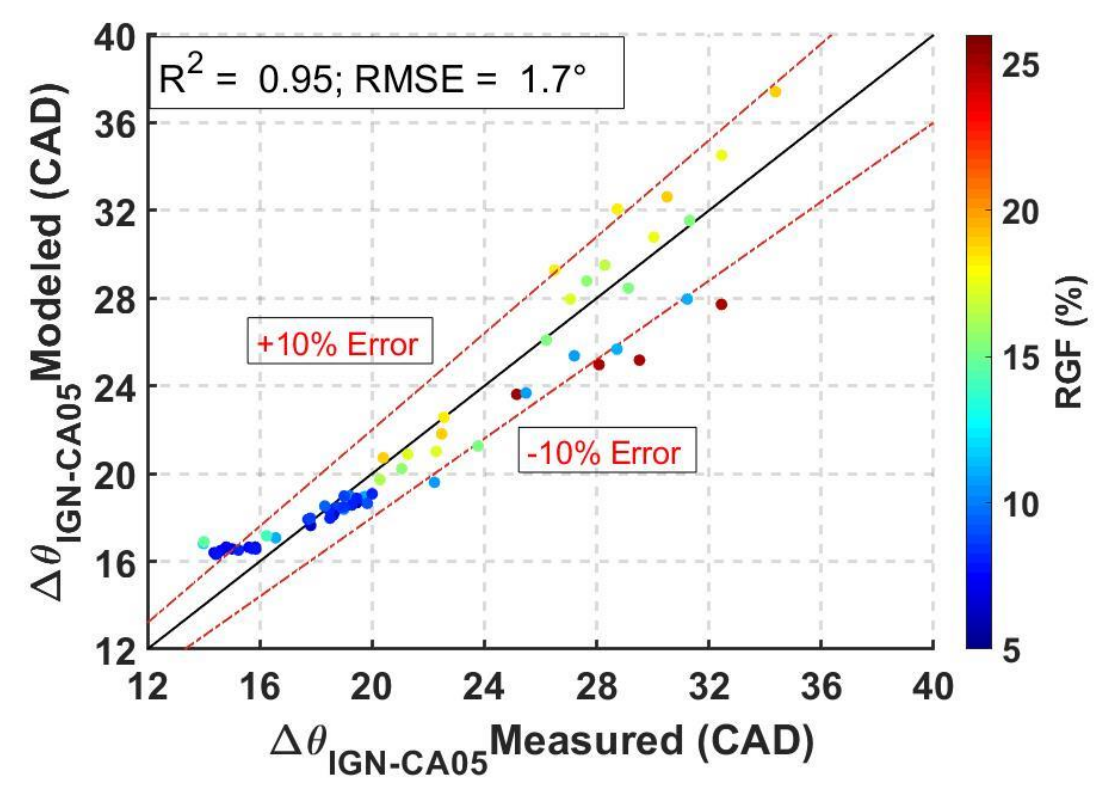

Figure 3.3 Modeled flame developed burn duration vs. experimental flame development duration

\subsubsection{Rapid Burning Stage Model}

In Equation (3.8), both the first term and the second term are similar and use the same physical parameters calculated at different crank angle locations to model flame development period and rapid burning period respectively. To further simplify the model and reduce calibration efforts, the correlation between experimental flame development 
period, $\Delta \theta_{I G N_{-} C A 05}$, and rapid burn burning period, $\Delta \theta_{C A 05_{-} C A 50}$, is examined to see if the prediction of $\Delta \hat{\theta}_{I G N-C A 50}^{-}$can be estimated as a function of $\Delta \hat{\theta}_{I G N-C A 05}^{-}$.

From the experimental optical study of SI engine combustion by Toulson [117], the flame radius is shown to increase linearly versus crank angle between ignition timing and 30 degrees after ignition timing. This phenomenon exists under different engine speeds/loads operating conditions and various lean combustion levels. In Irimescu's study [118], the linear correlation between flame area and volume fraction burned has been found under various engine speed conditions. From the experimental studies conducted by Robinet and Higelin [119], a linear correlation of $\Delta \theta_{I G N_{-} C A 05}$ vs. $\Delta \theta_{C A 05_{-} C A 10}, \Delta \theta_{I G N_{-} C A 10}$ vs. $\Delta \theta_{C A 10_{-} C A 20}$, and $\Delta \theta_{I G N_{-} C A 20}$ vs. $\Delta \theta_{C A 20_{C} C A 50}$ has been found.

Based on experimental findings from above studies [117-119], tests listed in Table 3.1 were analyzed to examine the correlation between $\Delta \theta_{I G N_{-} C A 05}$ and $\Delta \theta_{I G N_{-} C A 50}$. Figure 3.4 shows a linear relationship between experimental $\Delta \theta_{I G N_{-} C A 05}$ and $\Delta \theta_{I G N_{-} C A 50}$, with a correlation coefficient of 0.94 . The results show that $\Delta \hat{\theta}_{I G N-C A 50}^{-}$can be modeled using Equation (3.19).

$$
\Delta \hat{\theta}_{I G N-C A 50}^{-}=f\left(\Delta \hat{\theta}_{I G N-C A 05}^{-}\right)=a \cdot \Delta \hat{\theta}_{I G N-C A 05}^{-}+b
$$

Where, $a$ and $b$ are model coefficients. 


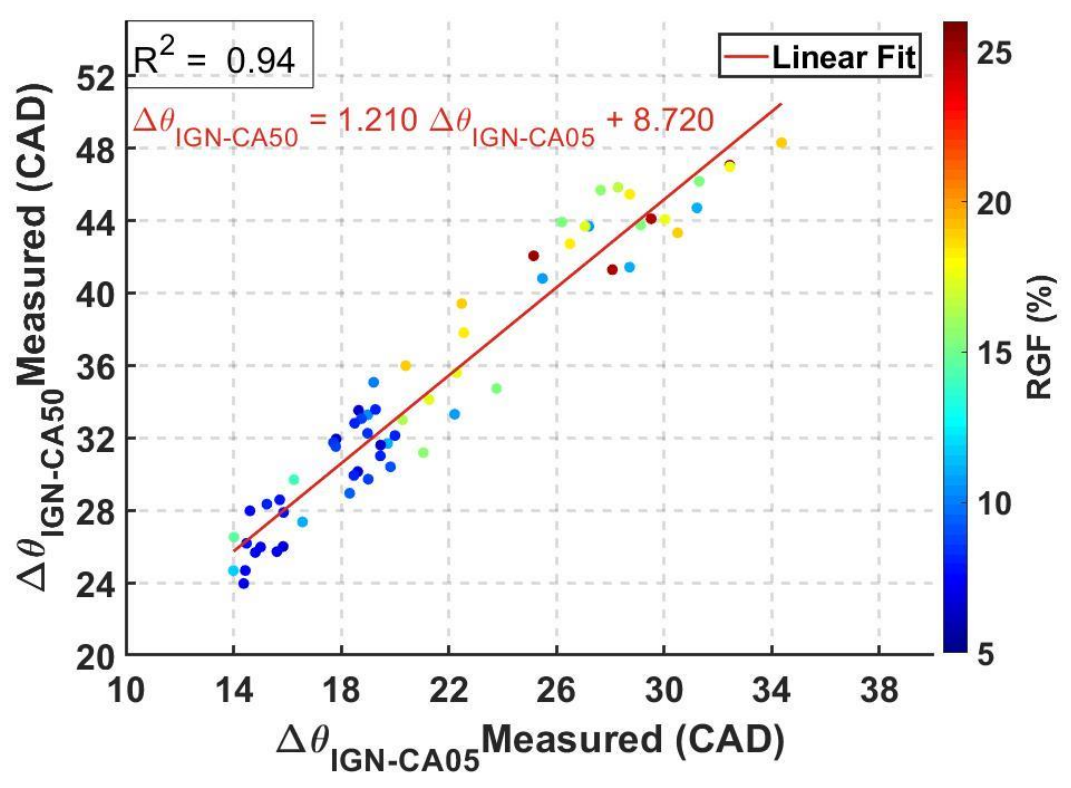

Figure 3.4 Experimental flame development period (IGN-CA05) vs. experimental rapid burning period (IGN-CA50)

Finally, the newly developed control-oriented combustion model for estimating the burn duration between ignition timing and CA50 is given in Equation (3.20). $T_{I G N}$ and $P_{I G N}$ represent the mixture conditions at ignition timing location and are affected primarily by three control inputs: throttle angle, cam phasing, and ignition timing. Engine speed, residual gas fraction, and lambda control the turbulent flame speed, which in turn impacts the burn duration. In Equation (20), there are seven parameters that need calibration $\left(a_{1}\right.$, $a_{7}$ ).

$$
\Delta \hat{\theta}_{I G N-C A 50}^{-}=a_{1} \cdot \frac{\left(P_{I G N}\right)^{a_{2}}}{\left(T_{I G N}\right)^{a_{3}}} \cdot \exp ^{\left(\frac{a_{4}}{T_{I G N}}\right)} \cdot\left(1+R G F+a_{5} \cdot \frac{\lambda-1}{\lambda+F A R_{\text {stoi }}}\right)^{a_{6}} \cdot R P M_{E n g}+a_{7}
$$


Model coefficients in Equation (3.20) were determined by minimizing the RMSE of $\Delta \widehat{\theta}_{I G N-C A 50}^{-}$using the 'fmincon' function in MATLAB. Figure 3.5 shows the results of modeled burn duration from ignition timing to CA50. A correlation coefficient of 0.94 indicates the control-oriented combustion model developed captures the dynamic changes of engine operating conditions. The RMSE of the model is $2.4 \mathrm{CAD}$, and $93 \%$ of the modeled burn duration falls within the $\pm 10 \%$ error lines. Table 3.2 shows the fitted model coefficients for Equation (3.20). Based on the fitted model coefficients shown in Table 3.2, it can be observed that increasing values of RGF and engine speed results in large burn duration prediction. $P_{I G N} / T_{I G N}$ represents the density of the unburned mixture, higher $P_{I G N} / T_{I G N}$ leads to decrease of burn duration prediction caused by the drop of laminar flame speed. Increasing $T_{I G N}$ results in decrease of $e^{a_{4} / T_{I G N}}$ and burn duration prediction. The trends of the modeled burn duration $\Delta \hat{\theta}_{I G N-C A 50}^{-}$match the trends of experimental burn duration caused by changes of engine operating conditions.

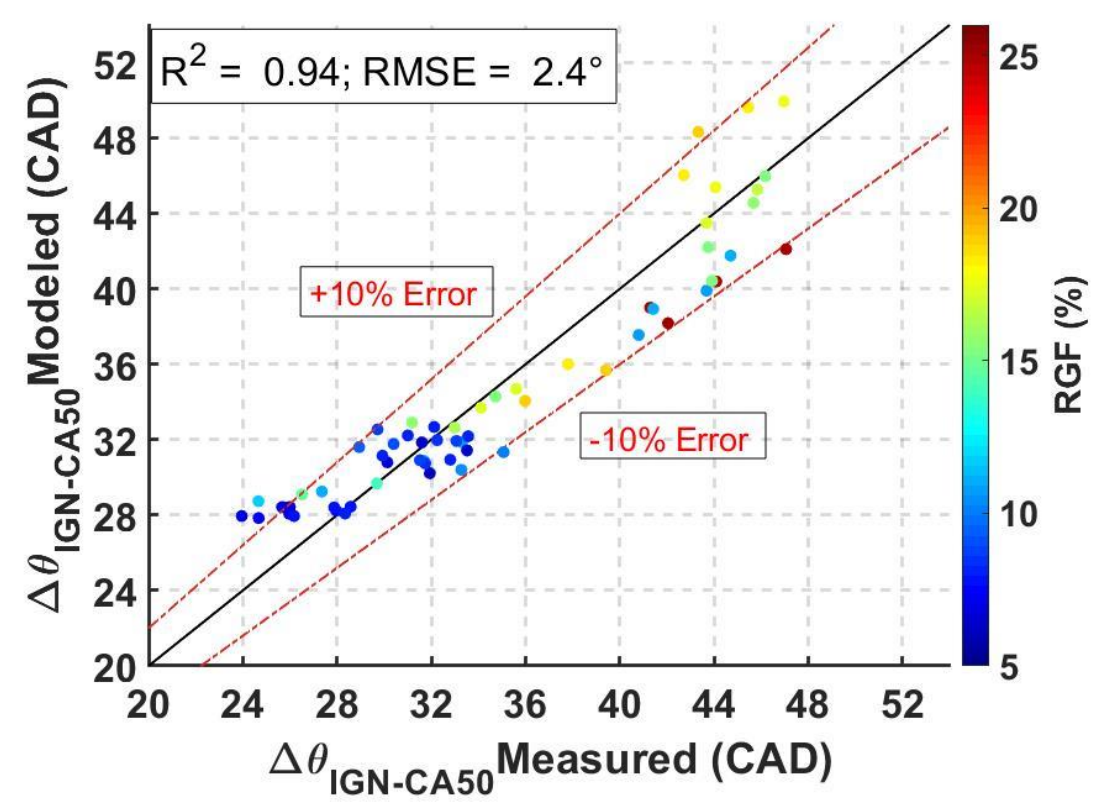

Figure 3.5 Experimental burn duration (IGN-CA50) vs. modeled burn duration (IGNCA50) 
Table 3.2 Fitted model coefficients

\begin{tabular}{|c|c|c|c|c|}
\hline Coefficients & $\boldsymbol{a}_{\mathbf{1}}$ & $\boldsymbol{a}_{\mathbf{2}}$ & $\boldsymbol{a}_{\mathbf{3}}$ & $\boldsymbol{a}_{\mathbf{4}}$ \\
\hline Value & $1.65 \mathrm{e}-12$ & -0.49 & -2.97 & 1256 \\
\hline Coefficients & $a_{5}$ & $a_{6}$ & $a_{7}$ & \\
\hline Value & 0.25 & 8.09 & 25.83 & \\
\hline
\end{tabular}

\subsubsection{Dynamic Burn Duration and Ignition Timing Prediction}

In order to achieve the ignition timing prediction for the model-based combustion phasing control purpose, the developed combustion model in Equation (3.20) is used to predict the burn duration based on the target CA50 cycle-by-cycle. Ignition timing can be calculated based on the predicted burn duration and desired CA50 target, $\theta_{\text {CA50_Target }}(k)$. Dynamic burn duration and ignition timing prediction algorithm is shown in Figure 3.6.

Cycle ' $\mathrm{k}-1$ ' is the past cycle and cycle ' $\mathrm{k}$ ' is the current cycle. Sensor information at and before IVC are needed for the algorithm. Calculated masses of residual gases and inducted fresh air trapped at IVC are used to calculate RGF for cycle ' $k$ '. Final ignition timing prediction for cycle ' $\mathrm{k}-1$ ' is used as the input to start the iterations to predict the ignition timing for the cycle ' $\mathrm{k}$ '. Each iteration checks the difference between $\hat{\theta}_{C A 50}^{-}(k, j)$ and $\theta_{C A 50 \_ \text {Target }}(k)$. If $\Delta \hat{\theta}_{C A 50}^{-}(k, j)$ is less than 0.5 CAD, the iteration is stopped. The threshold of the iteration number is set to ensure the safety of the real-time engine control. $\Delta \hat{\theta}_{I G N_{-} C A 50}^{-}(k)$ and $\Delta \hat{\theta}_{I G N}^{-}(k)$ are final predicted burn duration and ignition timing for the upcoming cycle ' $\mathrm{k}$ '. $\theta_{I G N}(k)$ is the final ignition timing sent to the engine ignition system. 


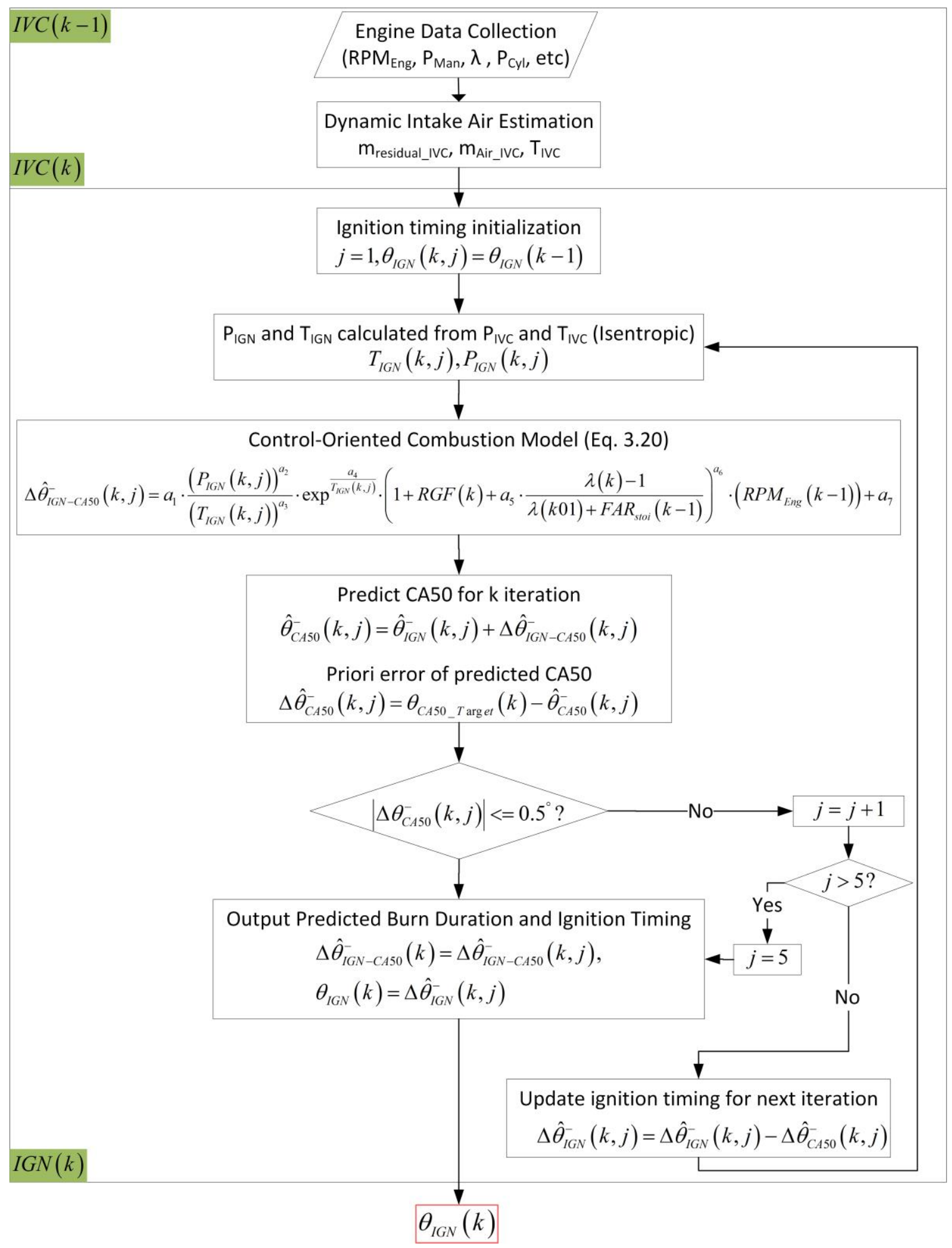

Figure 3.6 Dynamic Burn Duration and Ignition Timing Prediction 


\subsection{Experimental Validation}

Two sets of vehicle data recorded during track tests are used to examine the performances of model-based ignition timing prediction algorithm. The transient speed/load profiles were programmed into the dynamometer controller to duplicate the transient engine operating conditions tested in the vehicle by controlling the dyno speed and the pedal position. Both the production engine sensors and the instrumentation sensors described in Figure 2.2 were used to record the engine test data. The engine was auto-controlled by Bosch PCM. Table 3.3 shows the transient operating conditions regarding the acceleration and the deceleration calculated from dynamometer tests.

Table 3.3 Transient tests comparison

\begin{tabular}{|c|c|c|c|c|}
\hline & \multicolumn{2}{|c|}{$\begin{array}{c}\text { Max Speed Accel/Decel } \\
(\mathbf{r p m} / \mathbf{s})\end{array}$} & \multicolumn{2}{c|}{$\begin{array}{c}\text { Max Load Accel/Decel } \\
(\mathbf{b a r} / \mathbf{s})\end{array}$} \\
\hline Normal & 876 & -700 & 13 & -12 \\
\hline Heavy & 1372 & -1150 & 33 & -63 \\
\hline
\end{tabular}

Figure 3.7 shows the speed/load map of the normal transient test and details of engine acceleration/deceleration. Blue dots are engine operating points. Red dots represent 64 calibration points listed in Table 3.1. The entire transient test includes 3600 cycles of data. Figure 3.8 shows the speed/load map of the heavy transient test with rapid throttle opening and closing over 1050 cycles of recorded data. This heavy test has even wider engine operating conditions, where engine speeds/loads are from $1276 \mathrm{rpm} / 26 \mathrm{kPa}$ gross IMEP to $4000 \mathrm{rpm} / 1250 \mathrm{kPa}$ gross IMEP. In this test, $96 \%$ of cycles converge within 2 iterations. The average error of burn duration prediction is 1.5 CAD. 

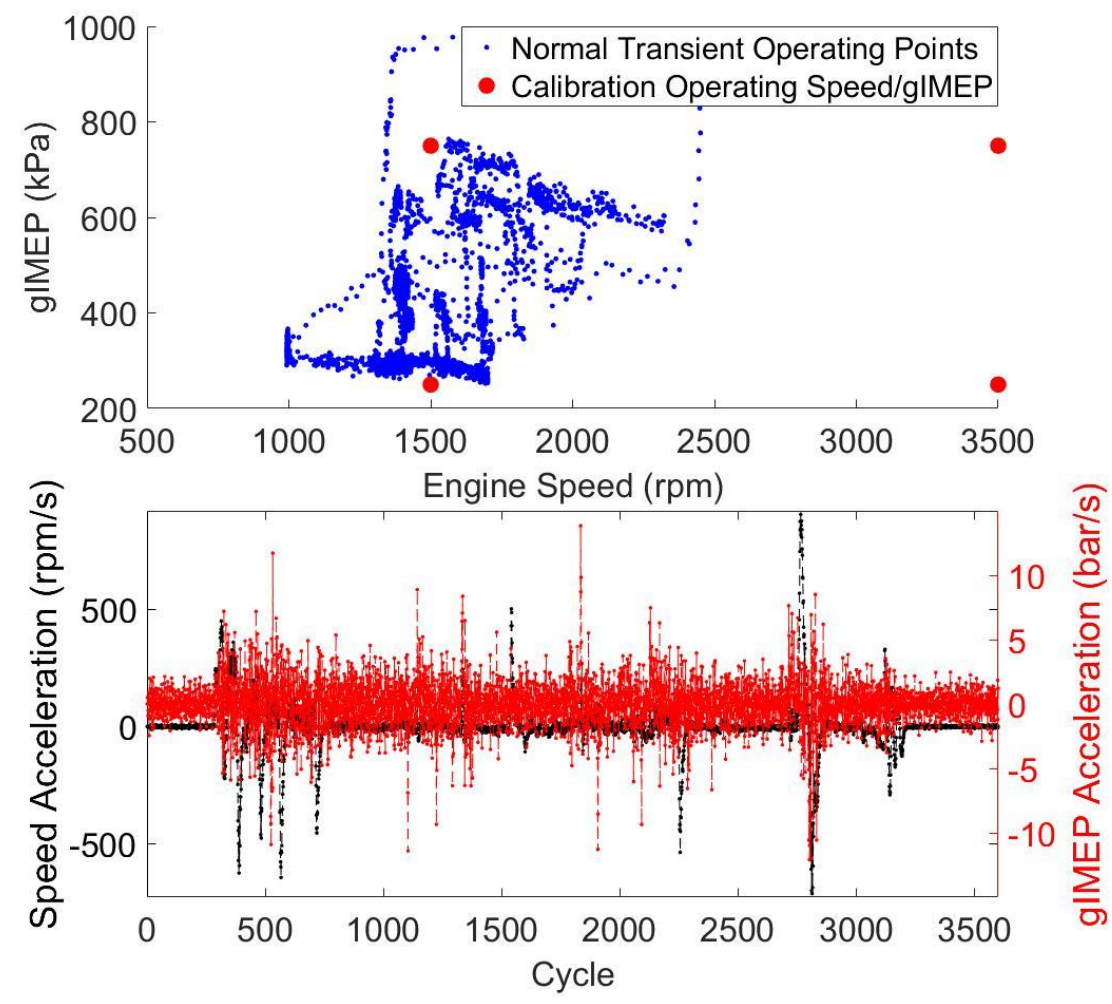

Figure 3.7 Normal transient test
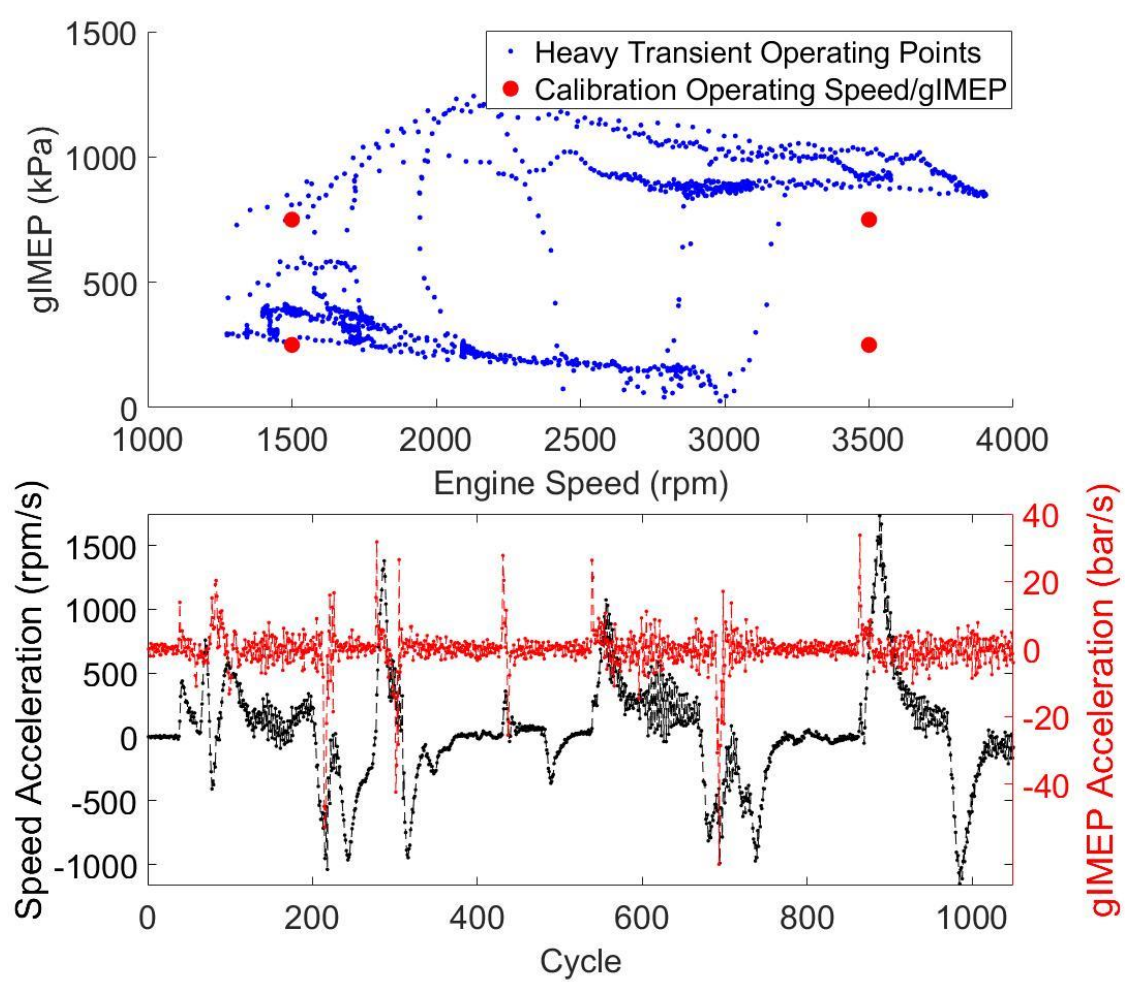

Figure 3.8 Heavy transient test 
Figure 3.9 shows performances of model-based burn duration and ignition prediction algorithm. The predicted burn duration follows the experimental burn duration, which changes due to the modified control inputs, including the throttle opening/closing, IVC advancing and EVO retarding. Most of the cycles only need 1 or 2 iterations, and none more than $3.50 \%$ of cycles converge within 1 iteration, and $49 \%$ of cycles need 2 iterations to converge. The burn duration prediction mean error is $-0.8 \mathrm{CAD}$. From the normal transient test, the developed combustion model can cover engine speeds/loads from 1000 $\mathrm{rpm} / 250 \mathrm{kPa}$ gross IMEP up to $2500 \mathrm{rpm} / 1000 \mathrm{kPa}$ gross IMEP with 64 calibration points.
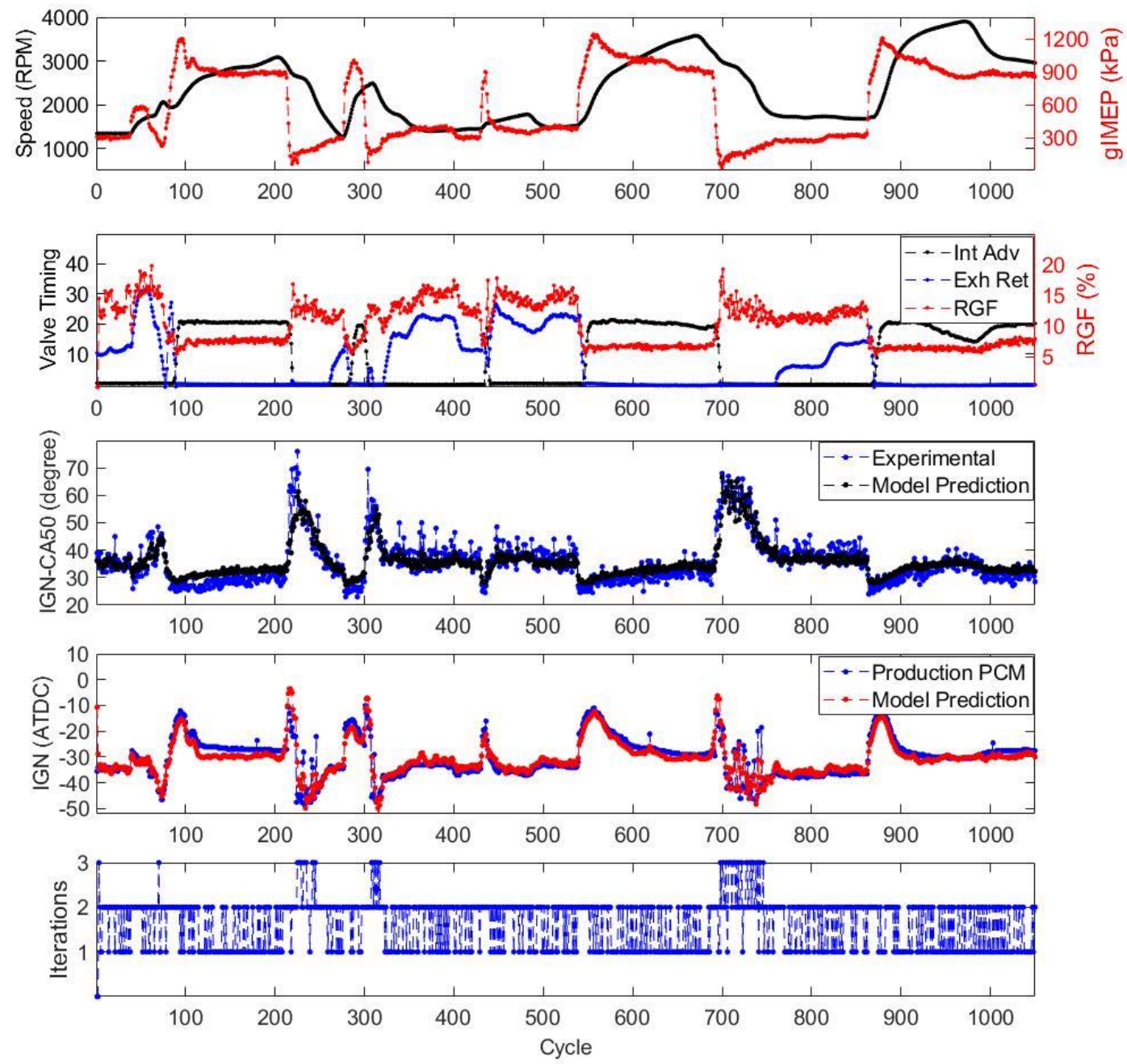

Figure 3.9 The performance of model-based burn duration and ignition prediction in a normal transient test 
Figure 3.10 shows the speed/load map of the heavy transient test with rapid throttle opening and closing over 1050 cycles of recorded data. This test has even wider engine operating conditions, where engine speeds/loads are from $1276 \mathrm{rpm} / 26 \mathrm{kPa}$ gross IMEP to 4000 $\mathrm{rpm} / 1250 \mathrm{kPa}$ gross IMEP. In this test, $96 \%$ of cycles converge within 2 iterations. The average error of burn duration prediction is 1.5 CAD.
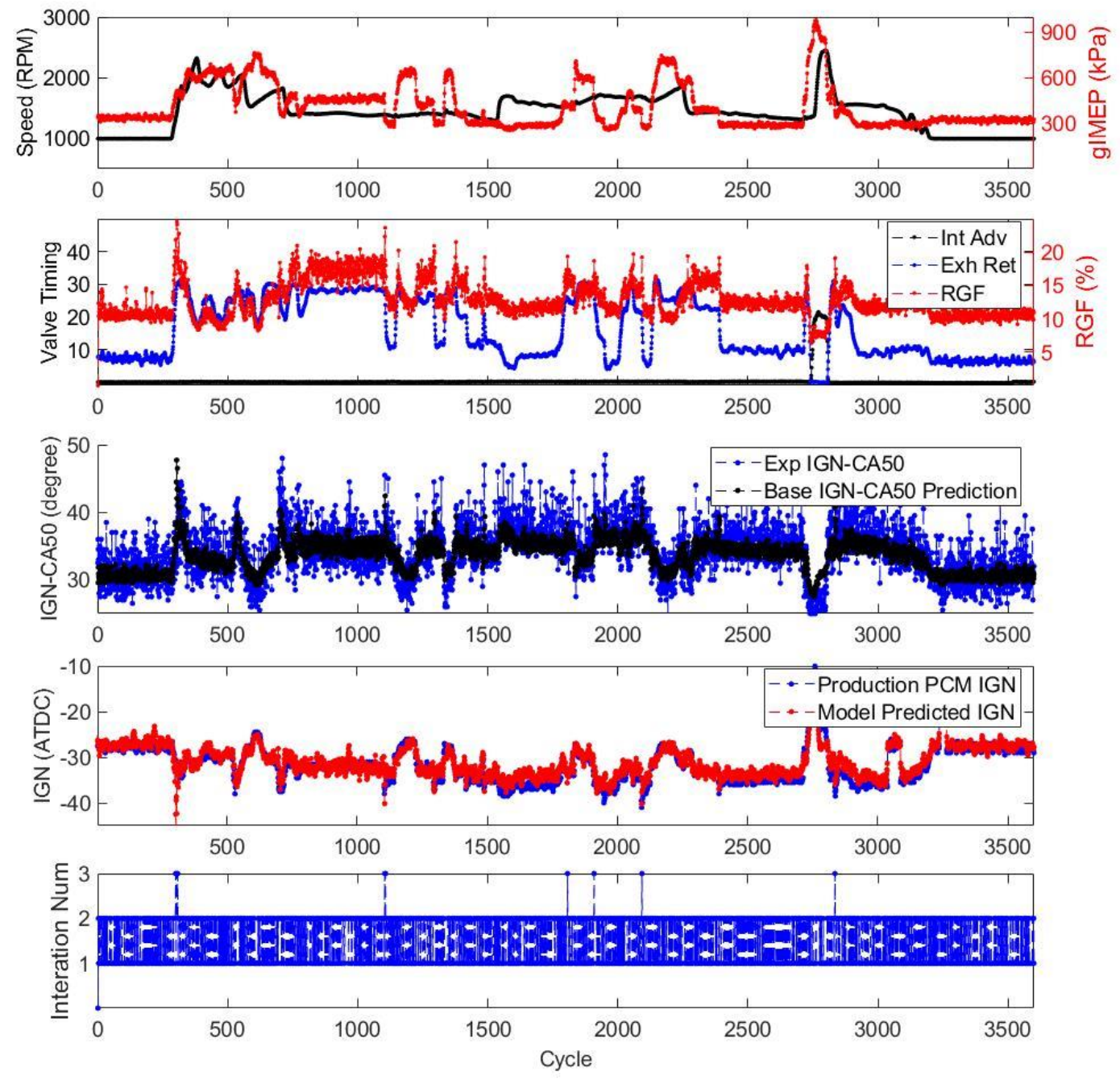

Figure 3.10 The performance of model-based burn duration and ignition prediction in a heavy transient test 
Figure 3.11 shows the prediction errors of burn duration and ignition timing combining two transient tests. The average prediction error of burn duration is $0.8 \mathrm{CAD} .83 \%$ of ignition timing prediction errors are within \pm 3 CAD. The STD of burn duration prediction error is 3.5 CAD and the STD of ignition timing prediction error is 2.4 CAD. Cycles with ignition prediction error exceeds $\pm 5 \mathrm{CAD}$ occur during tip in/out periods, like cycles between 200 and 250 in Figure 3.10, which are caused by the combination of stochastic cyclic combustion variations and model errors. An online model adaptation algorithm is developed to avoid large ignition timing prediction error during tip in/out periods in the section 4.1 .
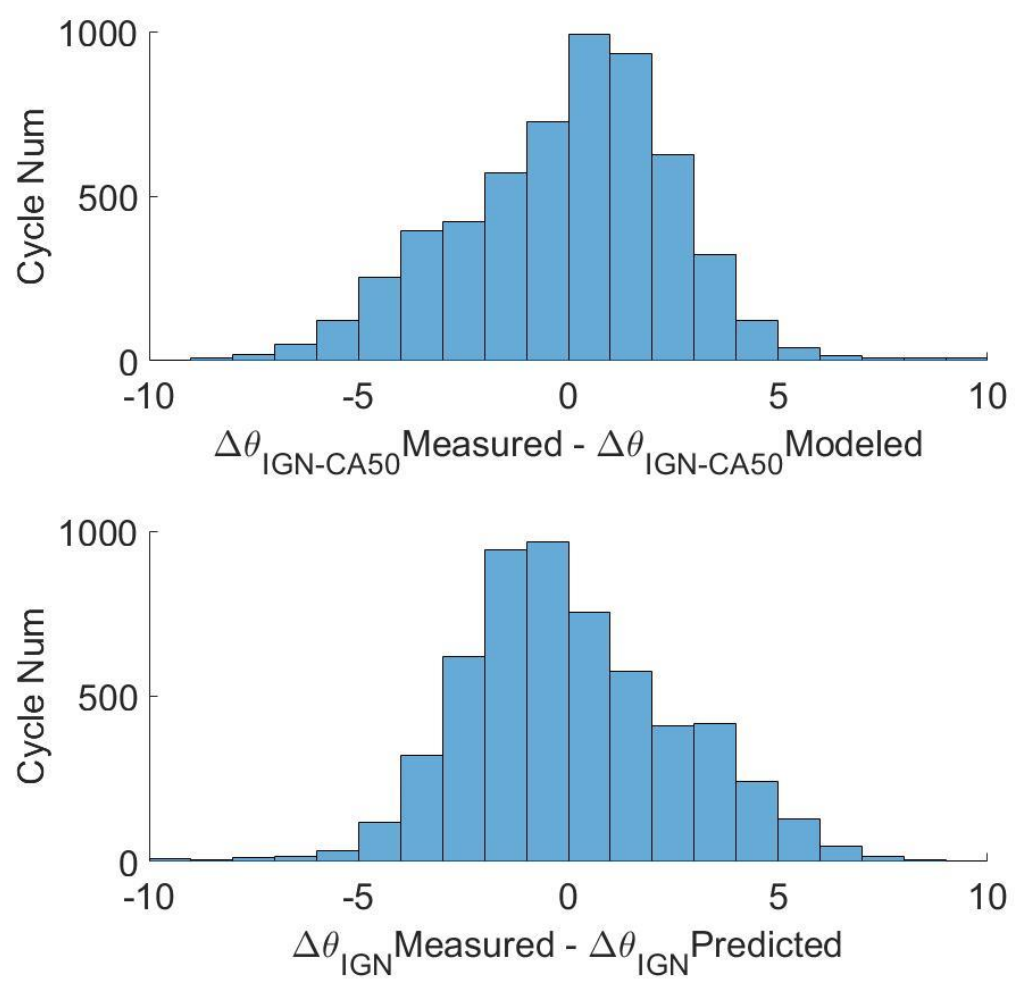

Figure 3.11 Error of burn duration and ignition timing prediction 


\subsection{Summary}

This work developed a new computationally efficient control-oriented burn duration prediction model. With only 64 calibration points, the combustion model can be used for a wide range of engine operating conditions, which contributes to shorten the engine development cycle. Trends of the modeled burn duration match the trends of experimental burn duration caused by changes of engine operating conditions. The RMSE of $\Delta \hat{\theta}_{I G N-C A 50}^{-}$ is $2.4 \mathrm{CAD}$, and the correlation coefficient is 0.94 .

The dynamic burn duration model and ignition timing prediction algorithm were proposed to predict the burn duration and the ignition timing at IVC for the upcoming cycle based on the desired CA50. From the results including transient tests, it can be observed that the algorithm needs no more than 3 iterations to converge and output the predicted ignition timing for the upcoming cycle based on target combustion phasing. $99 \%$ of cycles converge within 2 iterations in the normal transient test, and $96 \%$ of cycles converge within 2 iterations in the heavy transient test. Combining two transient tests, the average error of predicted ignition timing is $0.8 \mathrm{CAD}$. When compared to ignition timing stored in the Bosch PCM, 83\% of prediction errors of the model-based ignition timing prediction algorithm fall within $\pm 3 \mathrm{CAD}$. With the proper selection of the calibration point boundaries, the developed model-based burn duration and ignition timing prediction algorithm can be adapted to different engines and implemented into the engine ECUs for real-time combustion phasing control. 


\section{Adaptive Ignition Timing Management and Feedback Combustion Phasing Estimation for Combustion Phasing Control}

\subsection{Adaptive Model-Based Ignition Timing Management ${ }^{3}$}

To maintain the accuracy of developed combustion model and feedforward ignition timing management algorithm throughout the entire engine lifetime, a Recursive-Least-Square (RLS) with Variable Forgetting Factor (VFF) based adaptative algorithm is developed to handle operating-condition-dependent model errors. Due to the inherent model errors resulted from modeling assumptions and limited calibration points, the developed dynamic combustion model presented in Chapter 3 is expected to have operating condition dependent prediction errors. Even with the large amount of the steady state calibration points, the kind of model error cannot be omitted. Considering both not increasing the calibration burden with more dyno tests and ensuring the model accuracy within the entire engine operating region throughout the whole engine life, the on-board adaptative algorithm that can automatically adjust the model parameters through online learning to improve the model prediction accuracy becomes an optimal solution. Due to both operating-condition-dependent model prediction errors and stochastic characteristics of cycle-to-cycle combustion variations, large model errors may occur during severe transient operating conditions (tip-in/tip-out), which can result in wrong adjustments and excessive adaptations. Since on-road SI engines are always operating in transient conditions, the 'Heavy Transient Detection' algorithm is developed to avoid fault adaptation and assist the adaptative algorithm to be stable.

This chapter presents a recursive least square (RLS) based algorithm that can be easily implemented in the ECU to optimally calculate the adapted model parameters in real-time through online learning. For SI engines, cyclic variations of combustion duration/phasing exist all the time and different engine operating conditions have different levels of cyclic

\footnotetext{
3 The material contained in section 4.1 was previously published in the Proceedings of ASME 2019 Dynamic Systems and Control Conference, Volume 2, 2019 (See Appendix 1)
} 
variations [120]. These cyclic variations of combustion duration act as the 'outliers/noise' and corrupt the real combustion parameters used for online learning, which leads to misadjustments of adapted model parameters. As the SI engines in the vehicle are always operated under transient conditions, the cyclic 'outliers' have high potential to make the adaptative algorithm become unstable, especially under heavy transient operating conditions (rapid acceleration/deceleration). To reduce the negative effects of cyclic combustion variations on the adaptative algorithm, the self-tuning forgetting factor with real-time estimation of the level of cyclic variation has been integrated into the RLS algorithm. Besides, significant model prediction error may exist during heavy transient, which can result in over adjustment in the adapted model parameters. To avoid potential model errors under heavy transient conditions, the 'heavy transient detection' algorithm has been developed to ensure that the adaptative algorithm is stable and able to optimally derive the adapted model parameters automatically based on the engine operating conditions.

\subsubsection{Overview of Adaptive Model-Based Ignition Timing Management}

The complete structure of model-based GTDI SI engine control with adaptive ignition timing management is shown in Figure 4.1. This section focuses on the burn duration adaptation and adaptive ignition timing management in the feedforward path. The 'RLS Combustion Model Adaptation' block calculates adapted parameters used to compensate operating-condition-dependent model errors. 


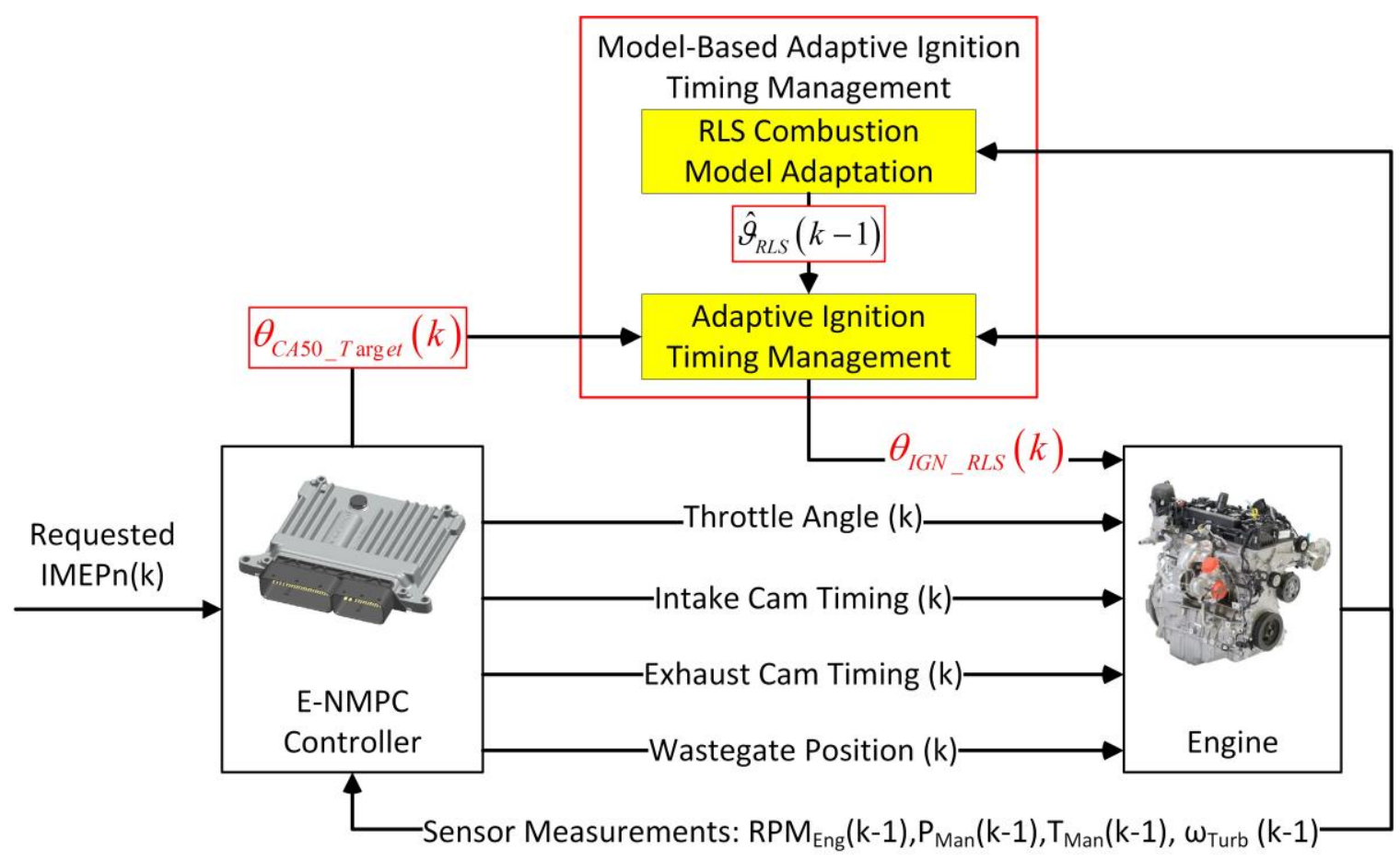

Figure 4.1 Structure of Model-Based GTDI SI Engine Control with Adaptive Ignition Timing Management

Figure 4.2 gives the detailed block diagram of the model-based adaptive burn duration and ignition timing management. The 'Dynamic Air Charge and Residual Gas Estimation' block provides estimated in-cylinder temperature and masses of fresh air and residual gases trapped at IVC. $\hat{\vartheta}_{R L S}$ is the adapted model parameters obtained by minimizing model errors between measured burn durations, $\Delta \theta_{\text {IGN_CA50 }}(k-1)$, and base model predictions, $\Delta \hat{\theta}_{I G N-C A 50}^{-}(k) . \theta_{I G N_{-} R L S}$ is the adapted ignition timing prediction calculated from the adapted burn duration, $\Delta \hat{\theta}_{(I G N-C A 50) \_R L S}^{-}(k)$, and the target combustion phasing, $\theta_{C A 50 \_ \text {Target }}(k)$. A dynamic burn duration and ignition timing prediction algorithm is developed to calculate both base burn duration predictions from the original controloriented combustion model and final adapted burn duration predictions. The 'Engine Operating Condition Detection' function generates a flag, 'Heavy_Transient_Flag(k)', to indicate whether the engine is under tip-in/tip-out operating conditions. This flag is used as an input to RLS-based combustion model adaptation algorithm. 

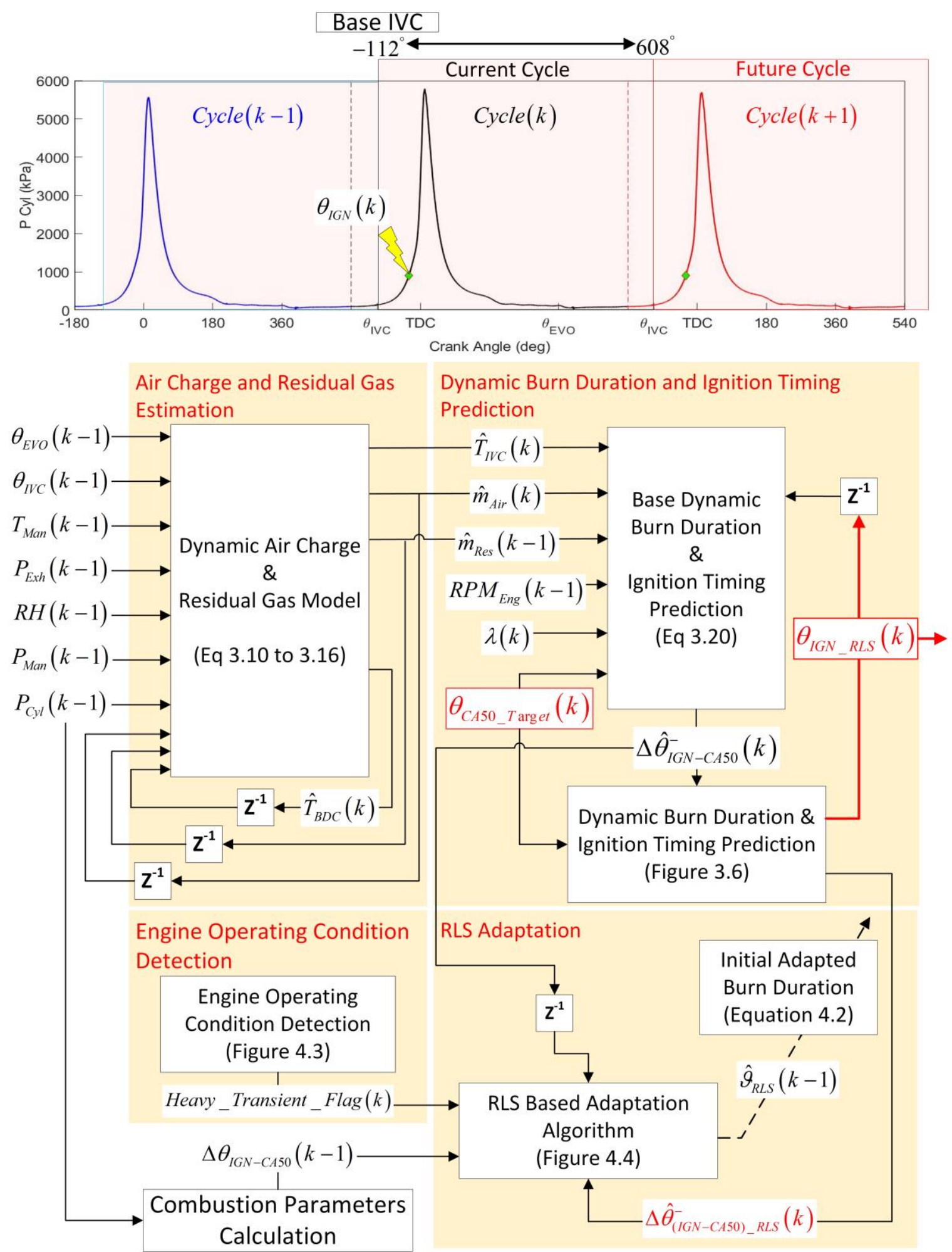

Figure 4.2 Block Diagram of Adaptive Ignition Timing Management 


\subsubsection{Recursive Least Square-Based Model Adaptation}

As previously discussed, the base control-oriented combustion model shown in Figure 3.5 is expected to have operating point dependent model errors with only 64 calibration points. In this section, the recursive least square method based online combustion model adaptation algorithm is developed to minimize operating-condition-dependent model errors without additional calibration efforts. Equation (4.1) describes measured burn durations, $\Delta \theta_{I G N_{-} C A 50}$, which can be described as the mean values of the combustion durations under steady state operating conditions corrupted by cyclic variations, $v$. Cyclic variations can be considered as 'stochastic noise or outliers' added to the actual combustion

duration. $\hat{\vartheta}_{R L S}$ represents the adapted parameters. $\varphi_{R L S}$ is the base control-oriented combustion model output, $\Delta \hat{\theta}_{I G N-C A 50}^{-} \cdot \Delta \hat{\theta}_{(I G N-C A 50) \_R L S}^{-}$is the adapted burn duration prediction, as described in (4.2). (4.3) defines the priori prediction error between measured burn duration and adapted burn duration. Since cyclic combustion variations are the nature of SI engines, minimizing the priori prediction error, $\varepsilon_{R L S}^{-}$, to reach zero will introduce wrong adaptations. The purpose of RLS-based combustion model adaptation is to find the optimal $\hat{\vartheta}_{R L S}$ to minimize the operating-point-dependent model errors without being corrupted by stochastic cyclic combustion variations.

$$
\begin{gathered}
y(k-1)=\overline{\Delta \theta}_{I G N-C A 50}(k-1)+v(k-1) \\
\hat{y}_{R L S}^{-}(k-1)=\Delta \hat{\theta}_{(I G N-C A 50)_{-} R L S}^{-}(k-1) \\
=\varphi_{R L S}^{T}(k-1) \cdot \hat{\vartheta}_{R L S}^{T}(k-2)=\Delta \hat{\theta}_{I G N-C A 50}^{-}(k-1) \cdot \hat{\vartheta}_{R L S}(k-2) \\
\varepsilon_{R L S}^{-}(k-1)=y(k-1)-\hat{y}_{R L S}^{-}(k-1)
\end{gathered}
$$


The forgetting factor governs the performance of the RLS adaptation algorithm. The RLS algorithm with Variable Forgetting Factor (VFF) has been widely studied and applied for system identification and online model adaptation [121]. Smaller forgetting factors improve the tracking ability but have higher potential of wrong adaptations and low adaptation stability. Forgetting factors that are close to 1 ensure the adaptation stability but lead to low tracking ability and converge rate of the algorithm. The purpose of using VFF is to ensure the tracking ability of the RLS adaptation algorithm without wrong adaptations caused by stochastic cyclic combustion variations. In this study, the VFF forgetting factor developed in [122] is used as the base VFF. Since cyclic combustion variations are dependent on engine operating conditions, a modified VFF, $\hat{\beta}_{R L S}$, is developed by incorporating online estimation of the level of cyclic variations, as described in Equation (4.4). $\hat{R}$ quantifies the level of cyclic combustion variation. The recursive method in [123] is modified and used for the online calculation of $\hat{R}$ cycle by cycle, as described in Equation (4.5). $\alpha_{R L S}$ is a weighting factor set to be 0.9. $\overline{\Delta \theta}_{I G N_{-} C A 50}$ is the mean value of measured burn duration from previous 10 cycles. $\widehat{F}_{R L S}$ is the adaptation gain used to calculate the adapted parameter, as shown in Equation (4.6). When cyclic combustion variations increase, $\hat{\beta}_{R L S}$ increases towards to 1 due to larger $\hat{R}$, resulting in smaller $\hat{F}_{R L S}$ and less adaptations. Larger model errors, $\varepsilon_{R L S}^{-}$, lead to decrease of $\hat{\beta}_{R L S}$, thus results in increase of $\widehat{F}_{R L S}$ and stronger adaptations. Equation (4.7) is used to calculate $\hat{\vartheta}_{R L S}$ cycle by cycle. In summary, large model errors result in smaller forgetting factors, thus improve the tracking ability by larger adaptation gains. Higher levels of cyclic variations result in larger forgetting factors, thus reduce adaptations. 


$$
\begin{gathered}
\hat{\beta}_{R L S}(k-1)=1-\frac{\left(\varepsilon_{R L S}^{-}(k-1)\right)^{2}}{\hat{R}(k-1)} \cdot\left(\frac{\varphi_{R L S}^{T}(k-1) \cdot \hat{F}_{R L S}(k-1) \cdot \varphi_{R L S}(k-1)}{1+\varphi_{R L S}^{T}(k-1) \cdot \hat{F}_{R L S}(k-1) \cdot \varphi_{R L S}(k-1)}\right) \\
\hat{R}(k-1)=\alpha_{R L S} \cdot \hat{R}(k-2)+\left(1-\alpha_{R L S}\right) \cdot\left(\Delta \theta_{I G N-C A 50}(k-1)-\overline{\Delta \theta}_{I G N-C A 50}(k-1)\right)^{2} \\
\hat{F}_{R L S}(k-1)=\frac{1}{\hat{\beta}_{R L S}(k-2)} \cdot\left(\hat{F}_{R L S}(k-2)-\right. \\
\left.\frac{\hat{F}_{R L S}(k-2) \cdot \varphi_{R L S}(k-2) \cdot \varphi_{R L S}^{T}(k-2) \cdot \hat{F}_{R L S}(k-2)}{\hat{\beta}_{R L S}(k-2)+\varphi_{R L S}^{T}(k-2) \cdot \hat{F}_{R L S}(k-2) \cdot \varphi_{R L S}(k-2)}\right) \\
\hat{\vartheta}_{R L S}(k-1)=\hat{\vartheta}_{R L S}(k-2)+\hat{F}_{R L S}(k-1) \cdot \varphi_{R L S}(k-2) \cdot \varepsilon_{R L S}^{-}(k-1)
\end{gathered}
$$

Most operating conditions of SI engines in the real world can be classified into two types. Heavy transient operating conditions are caused by sudden acceleration and deceleration with sudden throttle angle increase or decrease. The other type includes steady-state and medium transient operating conditions, which are caused by gradually open or close of the throttle. Since the combination of cyclic combustion variations, inaccuracy of in-cylinder pressure sensors, and operating-point-dependent model errors may result in large calculated model errors, which can drive the online adaptation to be unstable, especially under heavy transient operating conditions (tip-in/tip-out). It is not desirable that the model adaptation learns 'model errors' under the heavy transient conditions. To solve this issue, the 'Engine Operating Condition Detection' algorithm is proposed to distinguish the above two operating conditions, as shown in Figure 4.3. The change rate of the MAP signal is used to sense the engine operating conditions. Based on real vehicle driving tests, $10 \%$ MAP change rate is chosen to be the threshold to determine whether the engine enters the 'Heavy Transient'. When $P_{\text {Man_Rate }}$ is larger than $10 \%$, it means the engine is under tip-in conditions. When $P_{M a n_{-} \text {Rate }}$ is less than $-10 \%$, it indicates the engine is under tip-out conditions. 'Heavy_Transient_Flag=1' means the engine enters the 'heavy transient' condition. 'Heavy_Transient_Exit_Counter' is used to determine whether the engine exists 
the 'Heavy Transient' conditions by continually checking $P_{\text {Man_Rate }}$ for 20 cycles when 'Heavy_Transient_Flag' is set to be 1. The RLS algorithm selects the different variable forgetting factor based on the engine operating conditions indicated by 'Heavy_Transient_Flag'.

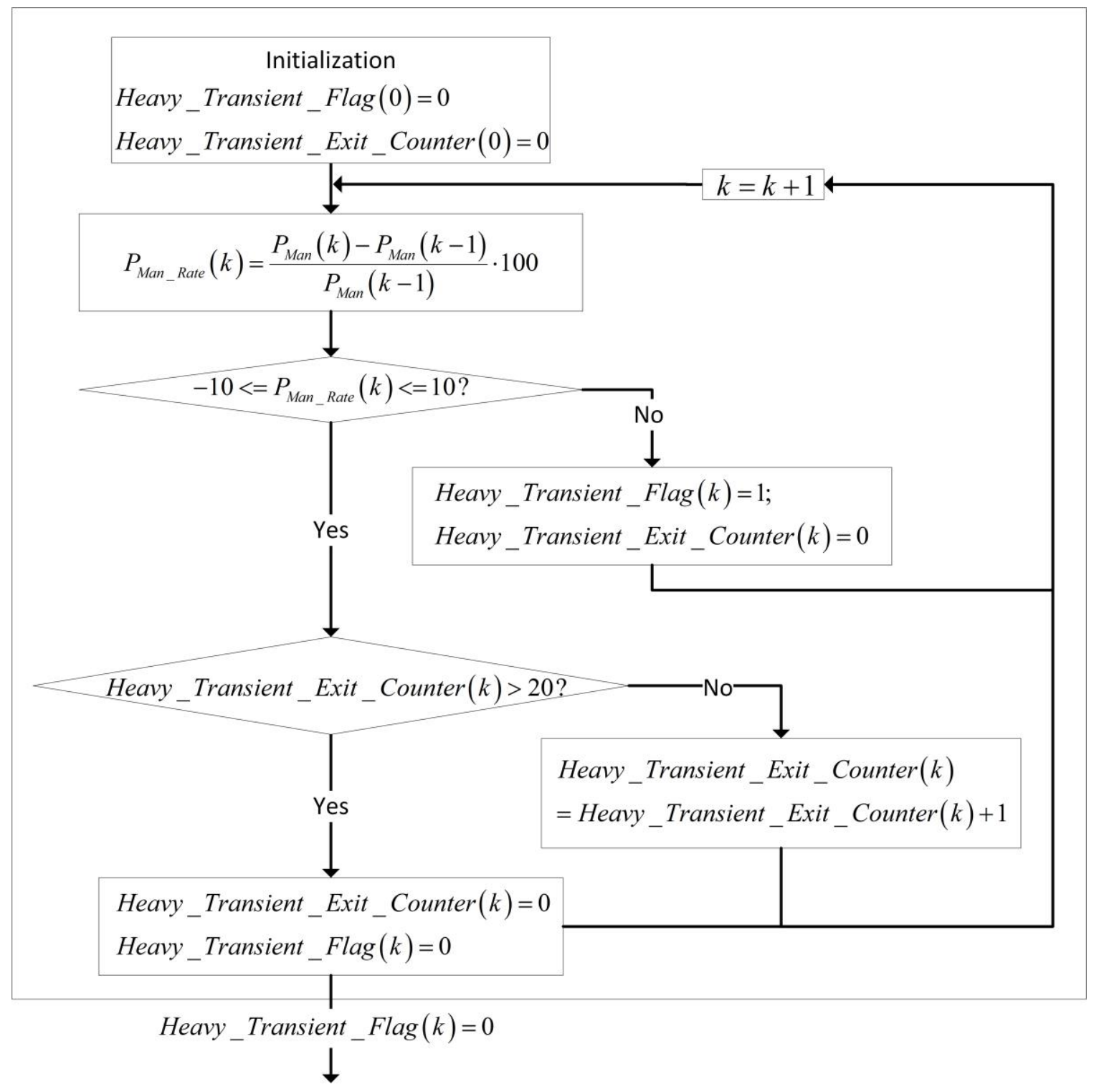

Figure 4.3 Engine Operating Condition Detection 
Figure 4.4 shows the complete diagram of VFF-RLS based combustion model adaptation algorithm. When the 'heavy transient' condition is detected, the variable forgetting factor is set to be 1 , which results in the minimum model adaptation gain. Under this condition, the adapted parameter, $\hat{\vartheta}_{R L S}$, has the minimum modification. When the engine is not under 'Heavy Transient' operating conditions, forgetting factors are calculated based on both base model prediction errors and the levels of cyclic combustion variations. Initial adapted burn duration is calculated using $\hat{\vartheta}_{R L S}$ and $\Delta \hat{\theta}_{I G N-C A 50}^{-}$, as described in Equation (4.2). The dynamic algorithm described in Figure 3.6 is applied to calculated adapted ignition timing based on the desired CA50. The 'Control-Oriented Combustion Model' block is substituted by Equation (4.2). 


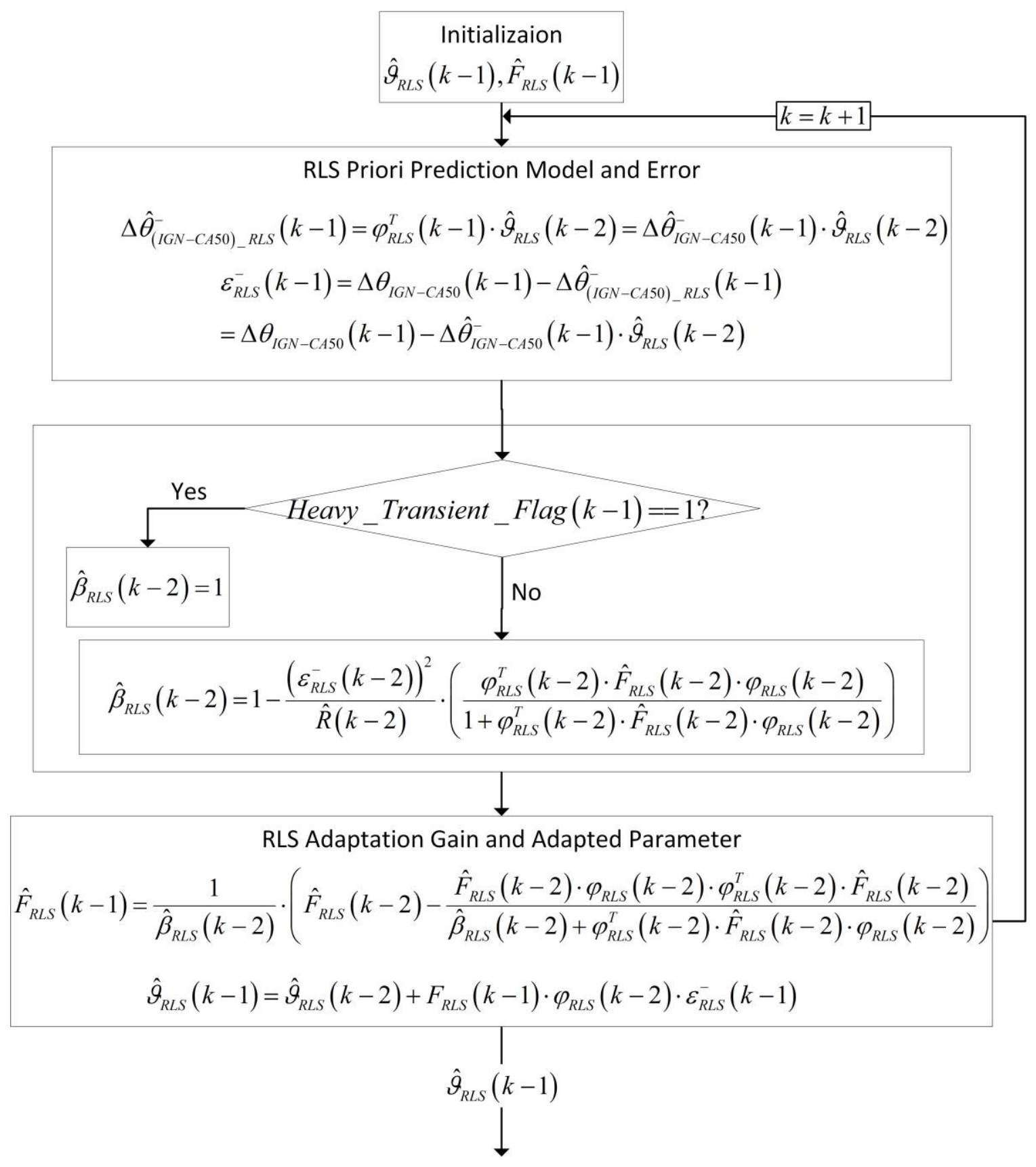

Figure 4.4 VFF-RLS Based Combustion Model Adaptation 


\subsubsection{RLS-Based Model Adaptation Experimental Validation}

Vehicle data recorded during track tests are used to examine the performances of controloriented combustion model-based adaptive burn duration and ignition timing prediction algorithm. Figure 4.5 shows the speed/load map of the heavy transient test. Blue dots are engine operating points. Red dots represent the speed/load range of 64 calibration points listed in Table 3.1. The test has wider engine operating conditions compared to the range of calibration points, where engine speeds/loads are up to $4000 \mathrm{rpm} / 1250 \mathrm{kPa}$ gross IMEP. Table 4.1 shows the acceleration and deceleration conditions of the transient test.

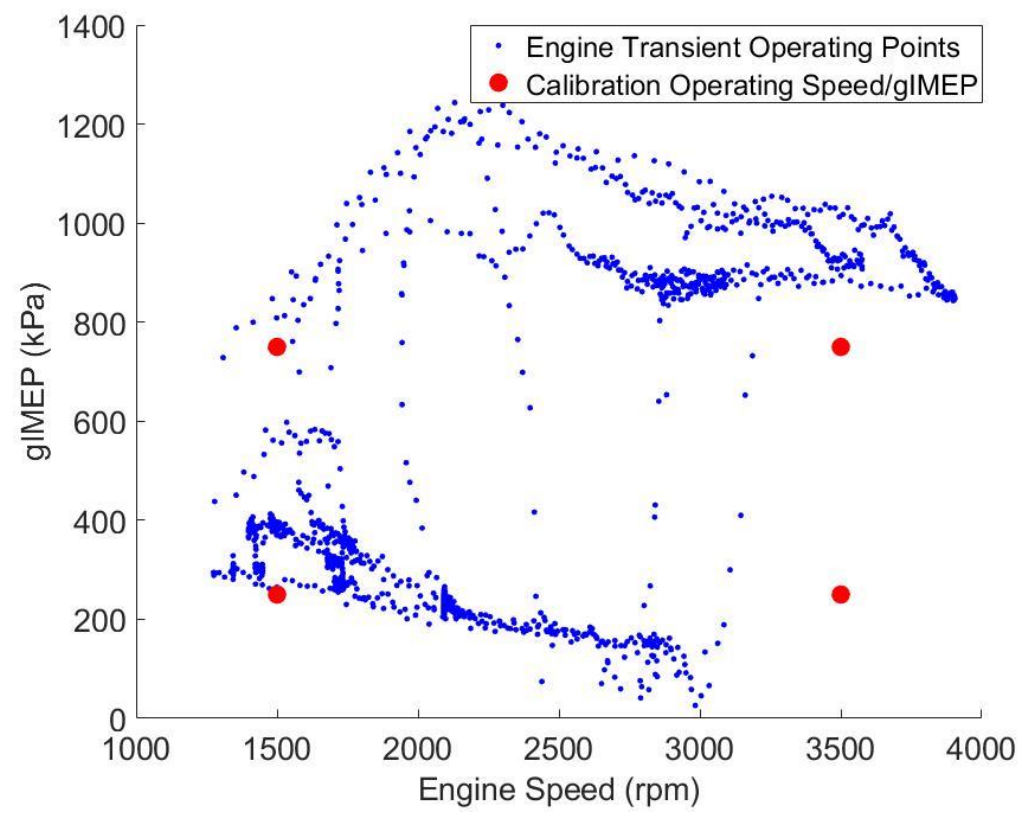

Figure 4.5 Transient Engine Operating Points

Table 4.1 Acceleration and Deceleration

\begin{tabular}{|c|c|c|}
\hline \multirow{2}{*}{ Tip-In } & $\begin{array}{c}\text { Max speed accel /decel } \\
(\mathbf{r p m} / \mathbf{s})\end{array}$ & $\begin{array}{c}\text { Max load accel/decel } \\
\text { (bar/s) }\end{array}$ \\
\hline \multirow{2}{*}{ Tip-Out } & 876 & 13 \\
\cline { 2 - 3 } & 1372 & 33 \\
\cline { 2 - 3 } & -700 & -12 \\
\hline
\end{tabular}


Figure 4.6 shows the detailed engine operating conditions of Figure 4.5. In Figure 4.6, the RGF value increases when the IVC timing advances or the EVC timing retards. Higher valve overlaps result in larger RGF. The estimated RGF changes due to the control inputs, including the throttle opening/closing, IVC advancing and EVO retarding. For 'Engine Operating Condition Detection' algorithm, it can be observed that all tip-in events result in $P_{\text {Man_Rate }}$ larger than $10 \%$ and all tip-out events leads to $P_{\text {Man_Rate }}$ less than $-10 \%$. All these tip-in/tip-out conditions caused by sudden open/close of the throttle will set 'Heavy_Transient_Flag' to be 1 for avoiding misadjustments calculated from the RLS adaptation algorithm. It can be observed that cycle 76 is detected to be the tip-in event by $P_{\text {Man_Rate }}$, and this is caused by continuous throttle opening from $6^{\circ}$ to $40^{\circ}$.

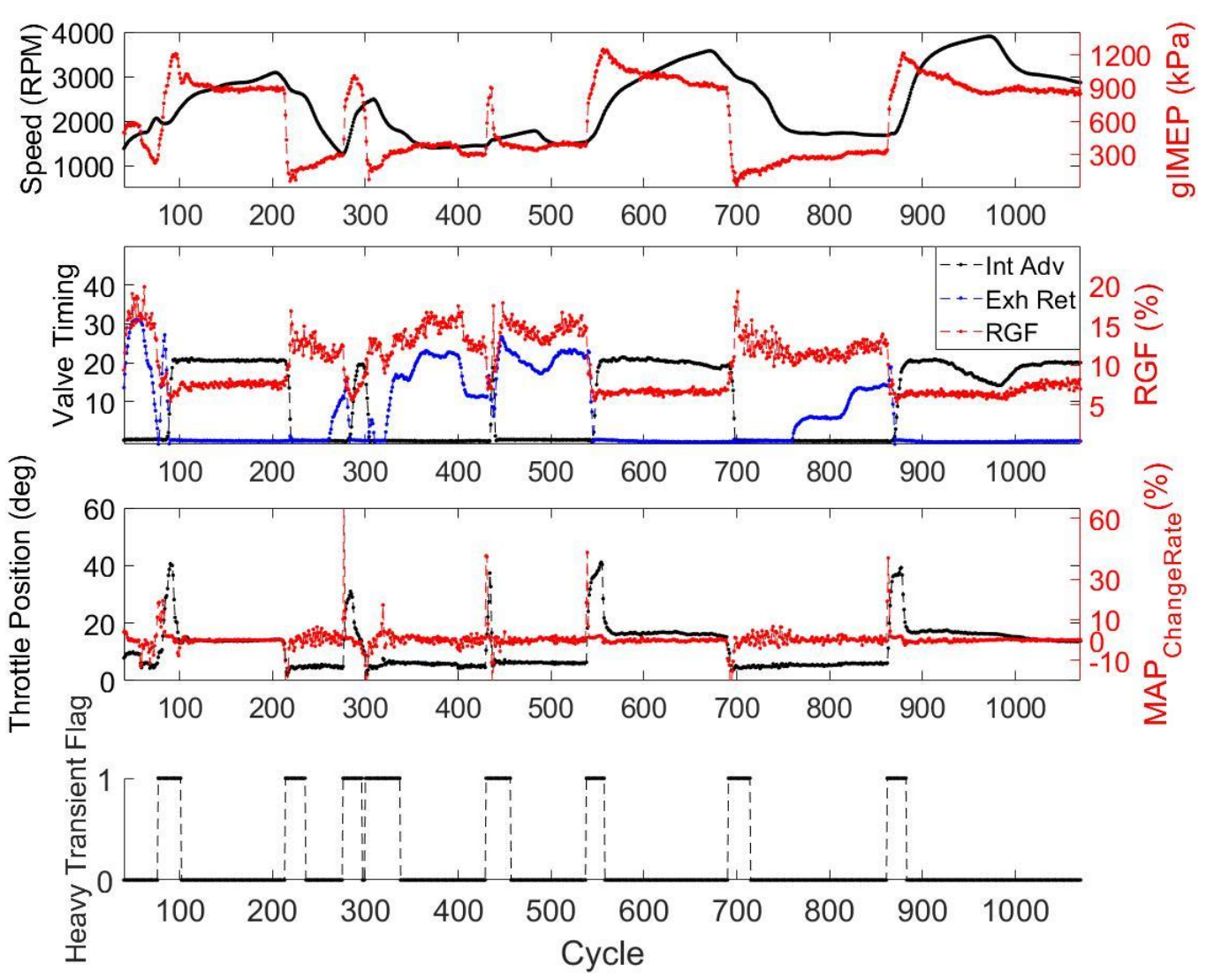

Figure 4.6 Transient Operating Condition 
Figure 4.7 shows the performances of both the base control-oriented combustion model and the RLS based adaptive burn duration and ignition timing prediction. In this test, $96 \%$ of cycles converge within 4 iterations and output the predicted ignition timing, combining the iteration numbers of both base ignition timing prediction and RLS based ignition timing prediction. $99 \%$ of cycles converge within 3 iterations. The average prediction errors of burn duration and ignition timing from the base model are 1.5 CAD and 1.4 CAD respectively. The average prediction errors of adaptive burn duration and adaptive ignition timing with the RLS algorithm are 0.1 CAD and 0 CAD respectively. Since cycle 76 is detected to be the tip-in event, 'Heavy_Transient_Flag' is set to be 1 from cycle 76 to cycle 100 and the forgetting factor is forced to be 1 during this tip in-period. From cycle 100 to cycle 146, the errors of burn duration prediction from the base control-oriented are larger than $3 \mathrm{CAD}$, and these make the forgetting factor to decrease, which drives the adapted parameter ' $\hat{\vartheta}_{R L S}$ ' from 1 to 0.86 . The adapted parameter ' $\hat{\vartheta}_{R L S}$ ' remains around 0.89 from cycle 146 to 216 , where the engine load is $860 \mathrm{kPa}$ gIMEP and engine speed gradually increase from 2800 to $3070 \mathrm{rpm}$. It takes 46 cycles to find the optimal $\hat{\vartheta}_{R L S}$ after engine enters steady-state conditions. This demonstrates that model adaptation with variable forgetting factor and 'Engine Operating Condition Detection' algorithm can derive the stable $\hat{\vartheta}_{R L S}$ without cyclic combustion variation effects under transient engine operating conditions. 

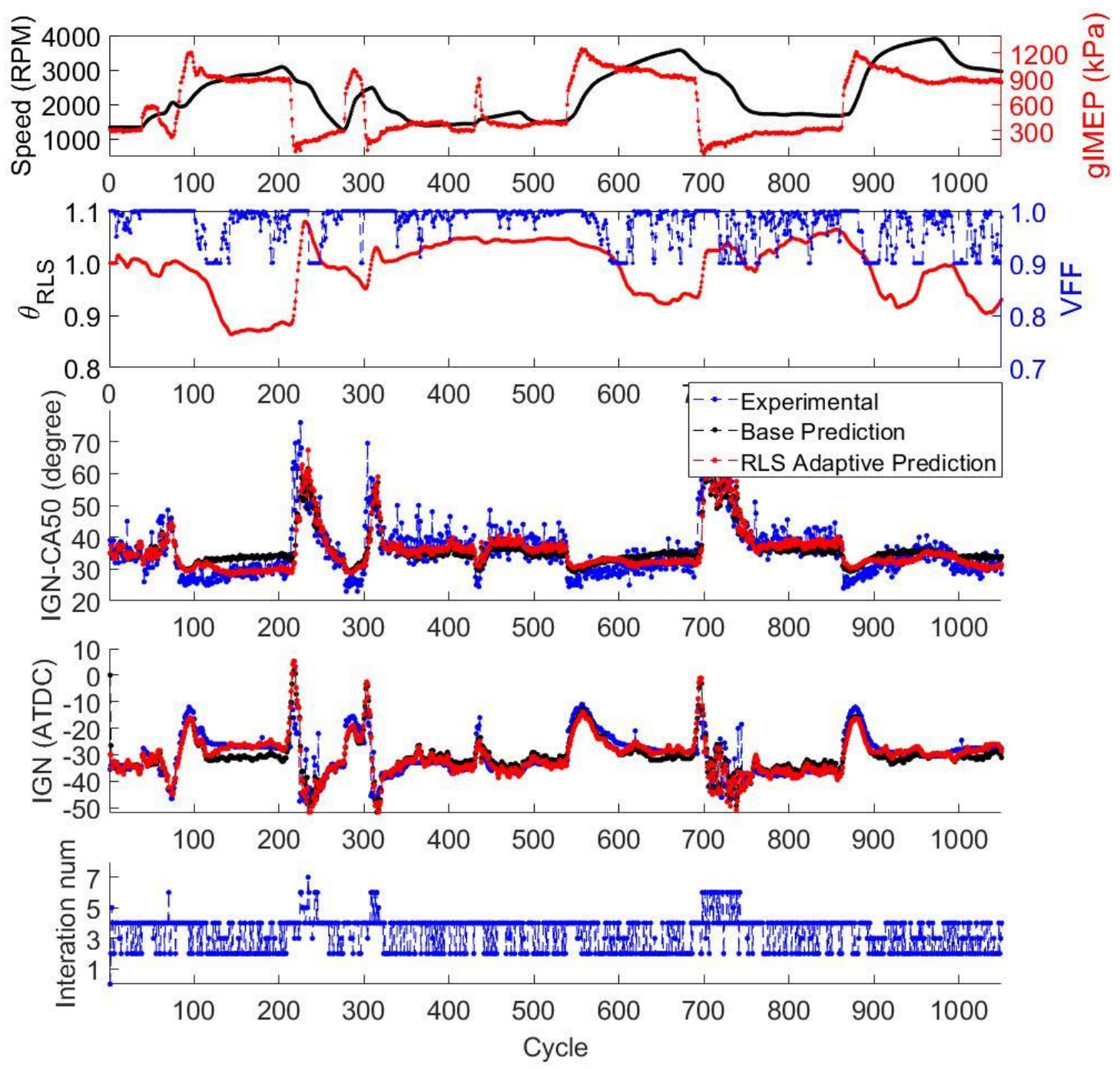

Figure 4.7 The Performance of Adaptive Burn Duration and Ignition Timing Prediction

Figure 4.8 shows the prediction errors of burn duration and ignition timing from both base control-oriented combustion model and adaptive combustion model. With RLS based adaptation, the average prediction error of burn duration drops from 1.5 CAD to 0.1 CAD, and the average prediction error of ignition timing drops from 1.4 CAD to 0 CAD. For base control-oriented combustion model, the STD of base burn duration prediction error is 3.5 CAD and the STD of base ignition timing prediction error is 2.4 CAD. For RLS based adaptive burn duration and ignition timing prediction, the STD of adaptive burn duration 
prediction error is 2.3 CAD and the STD of base ignition timing prediction error is 1.8 CAD.
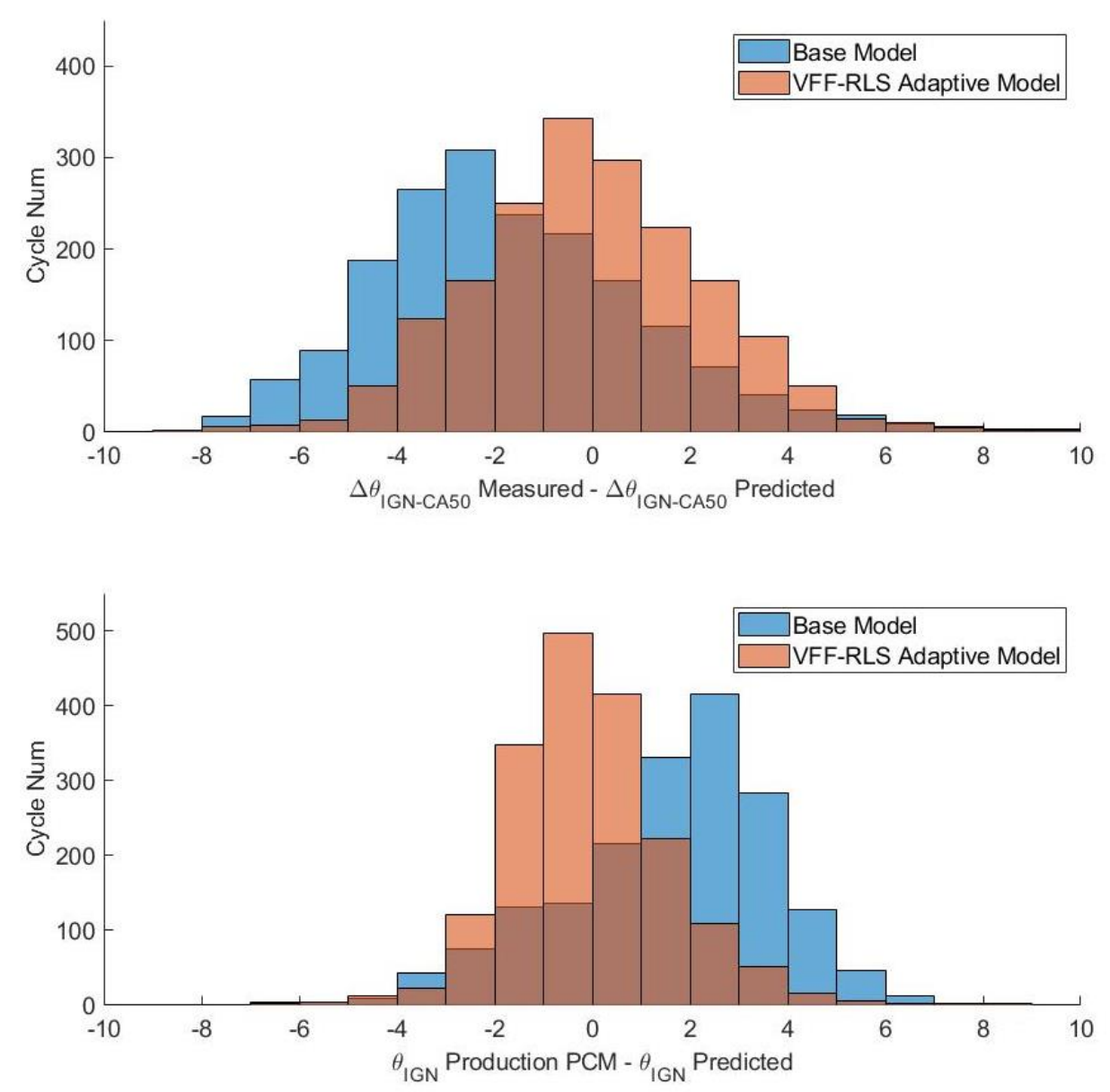

Figure 4.8 Errors of burn duration and ignition timing prediction

Online calculated $\hat{\vartheta}_{R L S}$ can be stored into the lookup table in the ECU memory, and the values can be updated in real-time. During the engine life cycle, the developed adaptation algorithm will improve the model accuracy, thus maintain the high engine efficiency and low emissions. Since $99 \%$ of cycles converge within 3 iterations, the algorithm of RLSbased adaptive burn duration and ignition timing prediction can be implemented into the engine ECU for the real-time combustion phasing control. 


\subsection{Adaptive Model-Based Combustion Phasing Estimation}

For the closed-loop combustion phasing control, the feedback estimation of combustion phasing has the key impact on the controller performance, including the stability, rising time, settling time, steady-state error, etc. Due to the nature of cyclic variations of SI engine combustion duration/phasing, the low pass filter and moving window average method are widely applied to obtain smooth and reasonably accurate combustion phasing estimation [124]. Significant cycle delays are introduced into the estimation when the large window size or low cutoff frequency are selected. The small window size cannot eliminate the cyclic 'outliers'. To overcome the negative effects of the above two shortcomings on the cycle-by-cycle feedback combustion phasing control, the extended Kalman filter (EKF) technique has been applied to CA50 feedback estimation [125-127]. Most of the EKF based CA50 observers use the fixed [126, 127] or pre-calibrated lookup tables [125] of covariance matrices of measurement noise and process noise, which were tuned through offline simulation using limited experimental data. The performances of the EKF are affected by two noise covariance matrices. Since the level of cyclic combustion variation depends on the engine operating conditions, the pre-calibrated values of covariance matrices may not be suitable for other engine operating conditions, and the improper values of noise covariance may make the EKF algorithm diverge. To solve the issues existing in conventional EKF based CA50 estimation, an adaptive extended Kalman filter (AEKF) has been developed to provide the feedback estimation of CA50, using the adaptive combustion

model developed. The developed ‘Engine Operating Condition Detection' algorithm and the forgetting factors are also integrated into the AEKF. 


\subsubsection{AEKF-based Feedback Combustion Phasing Estimation}

As the SI engines are operated under both steady-state and transient operating conditions, the accurate and fast estimation of combustion phasing feedback plays a key role in the closed-loop combustion phasing control. The actual combustion durations are corrupted by cyclic combustion duration variations, thus result in cyclic variations in CA50 estimation. The estimation algorithm should be able to minimize the effects of the stochastic components added to actual combustion durations. This work provides a method for fast CA50 estimation. An adaptive EKF method is used to estimate the feedback burn duration, and the estimated combustion duration is used to calculate the feedback CA50.

The model of burn duration estimation with AEKF is described in (4.8) . $\Delta \hat{\theta}_{(\text {IGN-CA50)_AEKF }}^{-}$ is the priori prediction of burn duration in AEKF. $w$ and $v$ are process noise and cyclic combustion variation respectively. $v$ represents cyclic combustion duration variation corrupted to the actual burn duration. $\hat{Q}_{A E K F}$ and $\hat{R}_{A E K F}$ are the covariance of process and measurement noise respectively. Since the burn duration is the only state, all parameters in the AEKF algorithm are scalars. Here, both $\mathrm{A}$ and $\mathrm{H}$ equal to 1.

$$
\begin{gathered}
\Delta \hat{\theta}_{(I G N-C A 50)_{-} A E K F}^{-}(k)=A \cdot \Delta \hat{\theta}_{(I G N-C A 50) \_R L S}^{-}(k)+w(k) \\
\Delta \theta_{I G N-C A 50}(k)=H \cdot \Delta \hat{\theta}_{(I G N-C A 50)_{-} A E K F}^{-}(k)+v(k) \\
\hat{Q}_{A E K F}(k)=\mathrm{E}[w(k) \cdot w(k)], \mathrm{E}[w(k)]=0 \\
\hat{R}_{A E K F}(k)=\mathrm{E}[v(k) \cdot v(k)], \mathrm{E}[v(k)]=0
\end{gathered}
$$

In this work, the AEKF algorithm introduced in [128] is used to estimate the feedback burn duration. The AEKF algorithm can be separated into two main steps, priori prediction, and post correction using measurements. 


\section{(1) Priori Prediction}

In this work, the adapted burn duration from the VFF-RLS algorithm is used to the priori prediction step, as shown in Equation (4.10). $\hat{P}_{A E K F}^{-}$in Equation (4.11) is the covariance of priori prediction.

$$
\begin{aligned}
& \Delta \hat{\theta}_{(I G N-C A 50)_{-} A E K F}^{-}(k)=A \cdot \Delta \hat{\theta}_{(I G N-C A 50)_{-} R L S}^{-}(k) \\
& \hat{P}_{A E K F}^{-}(k)=A \cdot \hat{P}_{A E K F}^{+}(k-1) \cdot A^{T}+\hat{Q}_{A E K F}(k-1)
\end{aligned}
$$

\section{(2) Post Correction}

In this step, the combustion duration is the existed information calculated from recorded cylinder pressure. Equation (4.12) is the priori prediction error of burn duration, where $\Delta \theta_{I G N-C A 50}$ is the feedback burn duration calculated from in-cylinder pressure trace. The feedback estimation of burn duration corrected by new measurements in equation (4.14) is calculated based on the priori prediction in equation (4.10), calculated Kalman gain in equation (4.13), and posteriori error in equation (4.15).

The performance of posteriori correction is heavily affected by the calculated Kalman gain. As can be seen from equation (4.13) and equation (4.11), both $\hat{Q}_{A E K F}$ and $\hat{R}_{A E K F}$ have a significant impact on $\widehat{K}_{A E K F}$. Normally, the try and error method is used to calibrate the covariance of the process noise and the measurement noise. The large lookup table was built in [125] for above two covariances to estimate CA50 by offline calibration. To reduce the calibration effort and the risk of divergence of the AEKF algorithm caused by improper values of above two covariances, the online estimation of both $\hat{Q}_{A E K F}$ and $\hat{R}_{A E K F}$ using the recursive method proposed in [129] and [128] is used in this work, as described in equation (4.16) and (4.17). $\alpha_{A E K F}$ is the forgetting factor and should be close to 1 . Here, $\alpha_{A E K F}$ is chosen to be 0.95 . The 'Engine Operating Condition Detection' algorithm is also applied 
to the AEKF algorithm. When the heavy transient operating conditions are detected, the $\alpha_{A E K F}$ is set to be 1 . Otherwise, $\alpha_{A E K F}$ is 0.95 . This helps avoid the divergence of the AEKF algorithm caused by actual large changes of combustion duration values during heavy transient conditions. The flowchart of AEKF based feedback estimation of the burn duration is described in Figure 4.9. Finally, the feedback CA50 estimation can be derived by equation (4.18).

$$
\begin{gathered}
\varepsilon_{A E K F}^{-}(k)=\Delta \theta_{I G N-C A 50}(k)-H \cdot \Delta \hat{\theta}_{(I G N-C A 50)_{-} A E K F}^{-}(k) \\
\hat{K}_{A E K F}(k)=P_{A E K F}^{-}(k) \cdot H^{T} \cdot\left(H \cdot P_{A E K F}^{-}(k) \cdot H^{T}+\hat{R}_{A E K F}(k)\right)^{-1} \\
\Delta \hat{\theta}_{(I G N-C A 50)_{-} A E K F}^{+}(k)=\Delta \hat{\theta}_{(I G N-C A 50) \_A E K F}^{-}(k)+\hat{K}_{A E K F}(k) \cdot \varepsilon_{A E K F}^{-}(k) \\
\varepsilon_{A E K F}^{+}(k)=\theta_{I G N-C A 50}(k)-H \cdot \Delta \hat{\theta}_{(I G N-C A 50)_{-} A E K F}^{+}(k) \\
\hat{R}_{A E K F}(k)=\alpha_{A E E K} \cdot \hat{R}_{A E K F}(k-1) \\
+\left(1-\alpha_{A E K F}\right) \cdot\left(\varepsilon_{A E K F}^{+}(k-1) \cdot\left(\varepsilon_{A E K F}^{+}(k-1)\right)^{T}+H \cdot P_{A E K F}^{-}(k) \cdot H^{T}\right) \\
\hat{Q}_{A E K F}(k)=\alpha_{A K E F} \cdot \hat{Q}_{A E K F}(k-1) \\
+\left(1-\alpha_{A K E F}\right) \cdot \hat{K}_{A E K F}(k) \cdot \varepsilon_{A E K F}^{+}(k) \cdot \varepsilon_{A E K F}^{+}(k) \cdot \hat{K}_{A E K F}(k) \\
\hat{\theta}_{C A 50 \_A E K F}^{+}(k)=\Delta \hat{\theta}_{(I G N-C A 50)_{-} A E K F}^{+}(k)+\theta_{I G N}(k)
\end{gathered}
$$




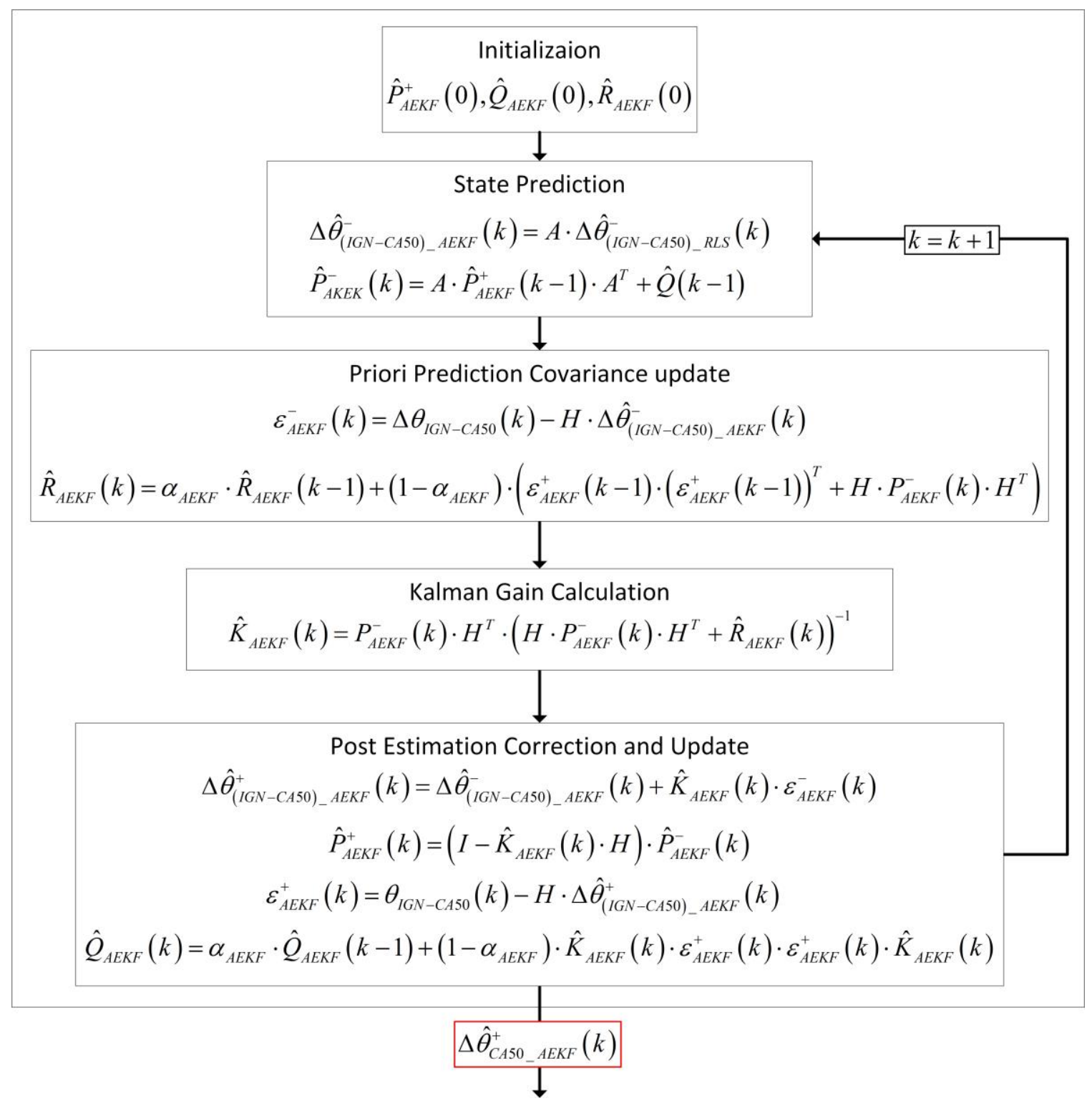

Figure 4.9 AEKF based Feedback Estimation of Burn Duration and CA50 


\subsubsection{Experimental Validation of AEKF-based Burn Duration and CA50 Estimation}

The heavy transient test data shown in Figure 4.5 were used to examine the proposed AEKF-based feedback estimations of both burn duration and combustion phasing. Figure 4.10 shows the results of the AEKF-based feedback estimations of both burn duration and CA50. The default value of the Kalman gain is set to be 0 in the beginning of the algorithm. The algorithm only takes 8 cycles to converge to the 'true' value, 0.35 . From the entire transient results, the AEKF-based estimation method successfully removes the large cyclic variation components from the raw data of both burn duration and CA50. With the assistance of adaptive burn duration model, the feedback estimation of IGN-CA50 has smooth and accurate responses under both heavy transient and medium transient/steadystate conditions. Equation (4.19) describes the moving average estimation of CA50, and $\theta_{C A 50}$ is the cyclic CA50 calculated from measured crank angle based in-cylinder pressure. Compared to 10 cycle moving average estimation of CA50, the AEKF-based method gives the same stable estimation of CA50 under near steady-state operating conditions. Under both the medium to heavy transient operating conditions, the AEKF-based method gives much faster response.

$$
\Delta \hat{\theta}_{C A 50 \_ \text {Moving }}(k)=\frac{1}{10} \cdot \sum_{d=1}^{10} \theta_{C A 50}(k-d+1)
$$



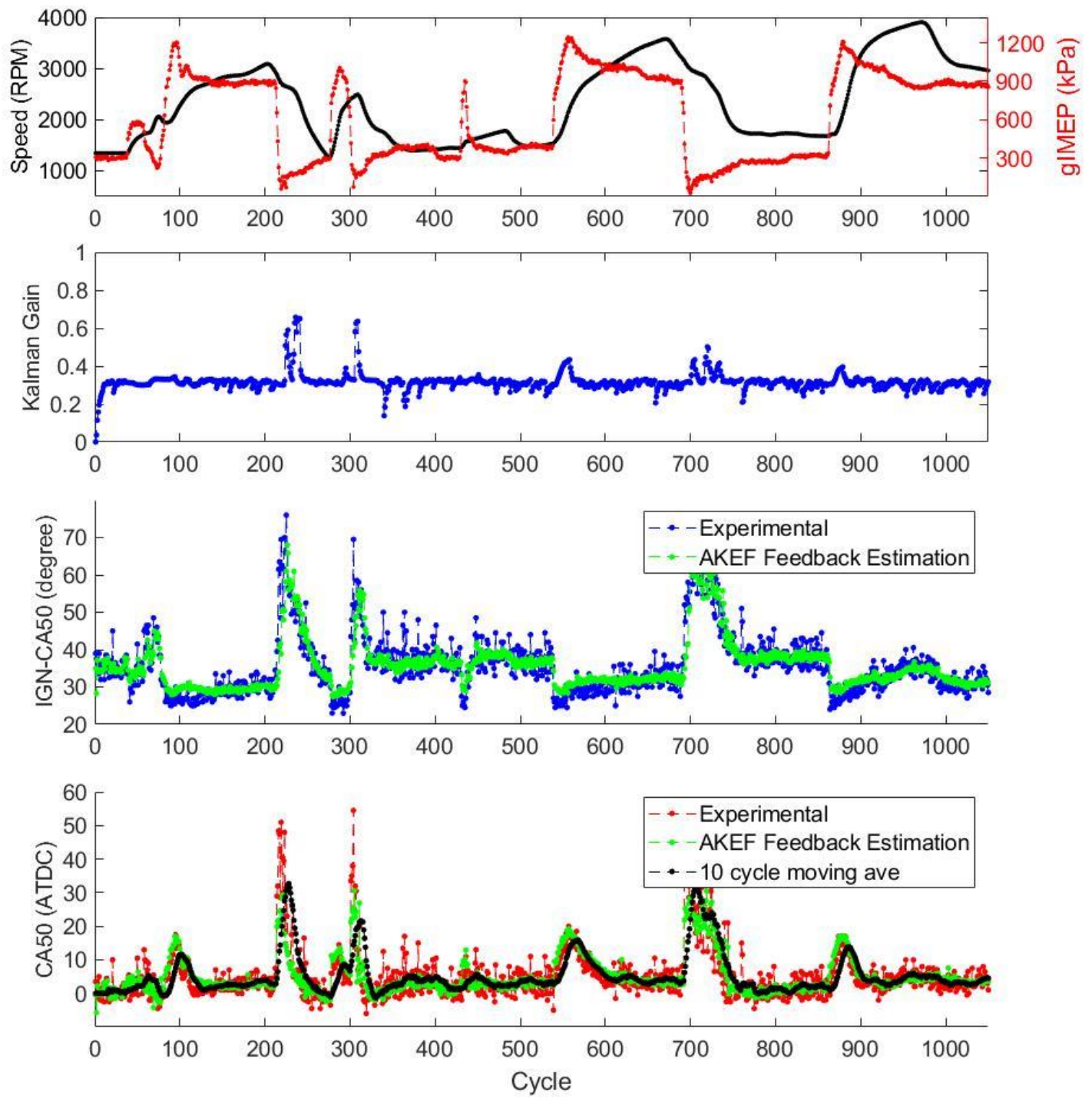

Figure 4.10 AEKF-Based Feedback Estimation of Burn Duration Estimation and Combustion Phasing 


\subsection{Summary}

This chapter develops a VFF-RLS based adaptive ignition timing management and AEKFbased burn duration and CA50 feedback estimation. The results demonstrate that VFF-RLS based adaptation method can improve the prediction accuracy of burn duration, and thus is able to provide more accurate feedforward ignition timing management for combustion phasing control. The variable forgetting factor considers both the level of cyclic variable existing in the SI combustion and base model error. From the transient test results, the VFF can quickly converge to the true value when there are large model errors. Under medium or near steady-state operating conditions, the VFF values are close to 1, which eliminate misadjustments caused by high 'stochastic noise' effect. With the proposed VFF-RLS based model adaptation technique, it further contributes to reducing the calibration effort and production development time. The derived values of adapted parameters can be stored into a lookup table in the ECU memory, and the values can be updated in real-time. During the engine life cycle, the developed adaptation algorithm will ensure the accurate model performance, thus maintain the high engine efficiency and low emissions.

Based on the stochastic characteristics of cycle-to-cycle combustion variation, a CA50 observer is proposed using an adaptive Extended Kalman Filter and developed combustion model. The performances of the developed adaptive prediction of burn duration and spark timing, and AEKF based CA50 estimation are evaluated using engine dyno test results under both steady-state and transient engine operating conditions. The AEKF algorithm successfully 'filters' out the 'stochastic noise' in the feedback signals. To reduce the calibration effort and the risk of divergence of the AEKF algorithm caused by improper selections of above two covariances in the EKF algorithm, the online estimation of the covariances of both process noise and measurement noise are implemented in this work. Compared to the traditional moving average based CA50 estimation, the developed AEKFbased CA50 estimation provides faster and more stable feedback CA50 signals, which is more suitable under transient engine operating conditions. 


\section{The Economic Nonlinear Model Predictive Control of GTDI SI Engine}

This chapter presents a model-based control system for cycle-by-cycle control of a Gasoline Turbocharged Direct Injection (GTDI) spark-ignition (SI) engine using an economic nonlinear model predictive controller. The presented E-NMPC engine control system is designed to solve a constrained optimal control problem (OCP) by minimizing a cost function with three objectives: delivery of requested net indicated mean effective pressure (IMEPn), minimization of fuel consumption, and reduction of NOx emissions. Since a turbocharged gasoline engine is an extremely complex system with nonlinear characteristics, both physics-based and data-driven modeling approaches have been employed to develop a control-oriented Multi-Input-Multi-Output GTDI SI engine model that is able to effectively and accurately predict the future engine behaviors and reduce the computational burden of E-NMPC for real-time engine control. The E-NMPC is implemented by using an open-source Automatic Control and Dynamic Optimization (ACADO) toolkit. The auto-coded $\mathrm{C}$ files are generated and integrated into the SIMULINK environment. The presented E-NMPC uses the Sequential Quadratic Programming (SQP) approach to generate an optimal sequence of control actions with the consideration of engine operating constraints, including both physical limitations of actuators and thresholds of abnormal combustion metrics comprising high variation of IMEP and combustion knock. A high-fidelity 1D GT-POWER GTDI SI engine model is also developed for the testing of the developed control system. The GT-POWER engine model is calibrated and validated by experimental test data. The performance of the E-NMPC engine control system has been evaluated through software-in-the-loop via co-simulation between GT-POWER and SIMULINK using on-road vehicle test data. The evaluation results show that the developed E-NMPC engine control system can track the driver's torque request and stably operate the engine while simultaneously reducing fuel consumption and NOx emissions without violating engine operating constraints. All these are achieved by systematically managing throttle position, spark timing, intake and exhaust 
valve phasing, and wastegate. Compared to baseline engine control, E-NMPC based engine control reduce the fuel consumption by $2 \%$ and reduce the NOx emission by $50 \%$ for a real-world driving cycle.

\subsection{Overview of Cycle-by-Cycle GTDI SI Engine Control using E- NMPC}

The presented model predictive control system is developed to control a GTDI SI engine as shown in Figure 5.1. The index ' $k$ ' indicates the $k t h$ cycle. The symbols of $P, T, m$, and $\omega$, represent pressure, temperature, mass, and shaft rotational speed, respectively. Five control signals are defined, including throttle opening angle $\left(\theta_{t h}\right)$, intake valve closing timing $\left(\theta_{I V C}\right)$, spark timing $\left(\theta_{I G N}\right)$, exhaust valve opening timing $\left(\theta_{E V O}\right)$, and wastegate opening percentage $\left(\theta_{W G}\right)$. The feedback signals used for E-NMPC are engine speed $\left(R P M_{E n g}\right)$, manifold temperature $\left(T_{M a n}\right)$ and manifold pressure $\left(P_{M a n}\right)$ from the T-MAP sensor, turbo speed $\left(\omega_{\text {Turb }}\right)$, and lambda $(\lambda)$ from the universal gas oxygen sensor (UEGO) sensor. The lambda value is used by the fuel injection control system to maintain the stoichiometric air-fuel ratio (AFR). The turbocharger related parameters including ' $c$ _in', 'c_out', 't_in', and 't_out', represent compressor inlet, compressor outlet, turbo inlet, and turbo outlet, respectively. $P_{T I P}$ is the throttle inlet pressure. $m_{t h}$ air represents the air mass flow through the throttle. $m_{c y l}$ air is the mass of the fresh air inducted into the cylinder. $\mathrm{KI}$ is the knock intensity. 


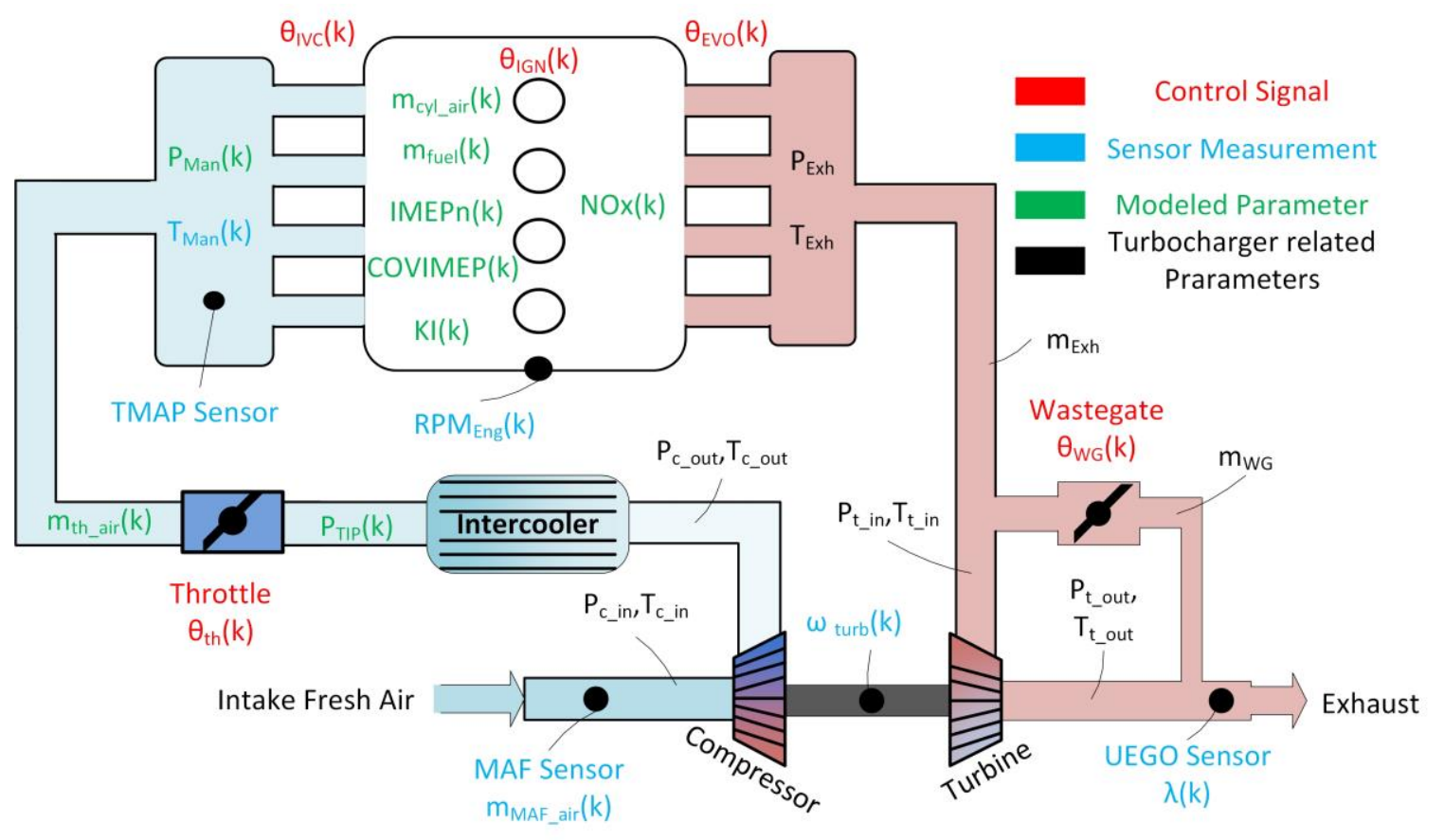

Figure 5.1 Schematic of GTDI SI Engine System

An overview of the model-based control system for the GTDI SI engine is shown in Figure 5.2. For every upcoming cycle within a given prediction horizon, the model-based engine control system finds optimal control actions to deliver the requested torque while minimizing fuel consumption $\left(m_{f u e l}\right)$ and NOx emission. From equation (5.1), the engine brake torque is a function of IMEPn and friction mean effective pressure (FMEP), which represents the actual friction (e.g, mechanical rubbing) and axillary losses. FMEP is not affected by control actions and usually tabulated or modeled as a function of engine speed and torque from experimental tests. This work uses IMEPn as a surrogate for torque and focuses on delivering the requested IMEPn, which is independent of engine displacement and represents the net indicated engine torque divided by the displacement.

$$
T_{\text {brake }}=\frac{V_{d} \cdot B M E P}{4 \cdot \pi}=\frac{V_{d} \cdot\left(I M E P_{n}-F M E P\right)}{4 \cdot \pi}=T_{n_{-} \text {ind }}-T_{f}
$$


Where,

Thrake Engine brake torque $(\mathrm{Nm})$

$V_{d} \quad$ Cylinder volume $\left(\mathrm{m}^{3}\right)$

$B M E P \quad$ Brake mean effective pressure $(\mathrm{Pa})$

FMEP Friction mean effective pressure $(\mathrm{Pa})$

$T_{n \_ \text {ind }} \quad$ Indicated net torque $(\mathrm{Nm})$

$T_{f} \quad$ Friction torque $(\mathrm{Nm})$
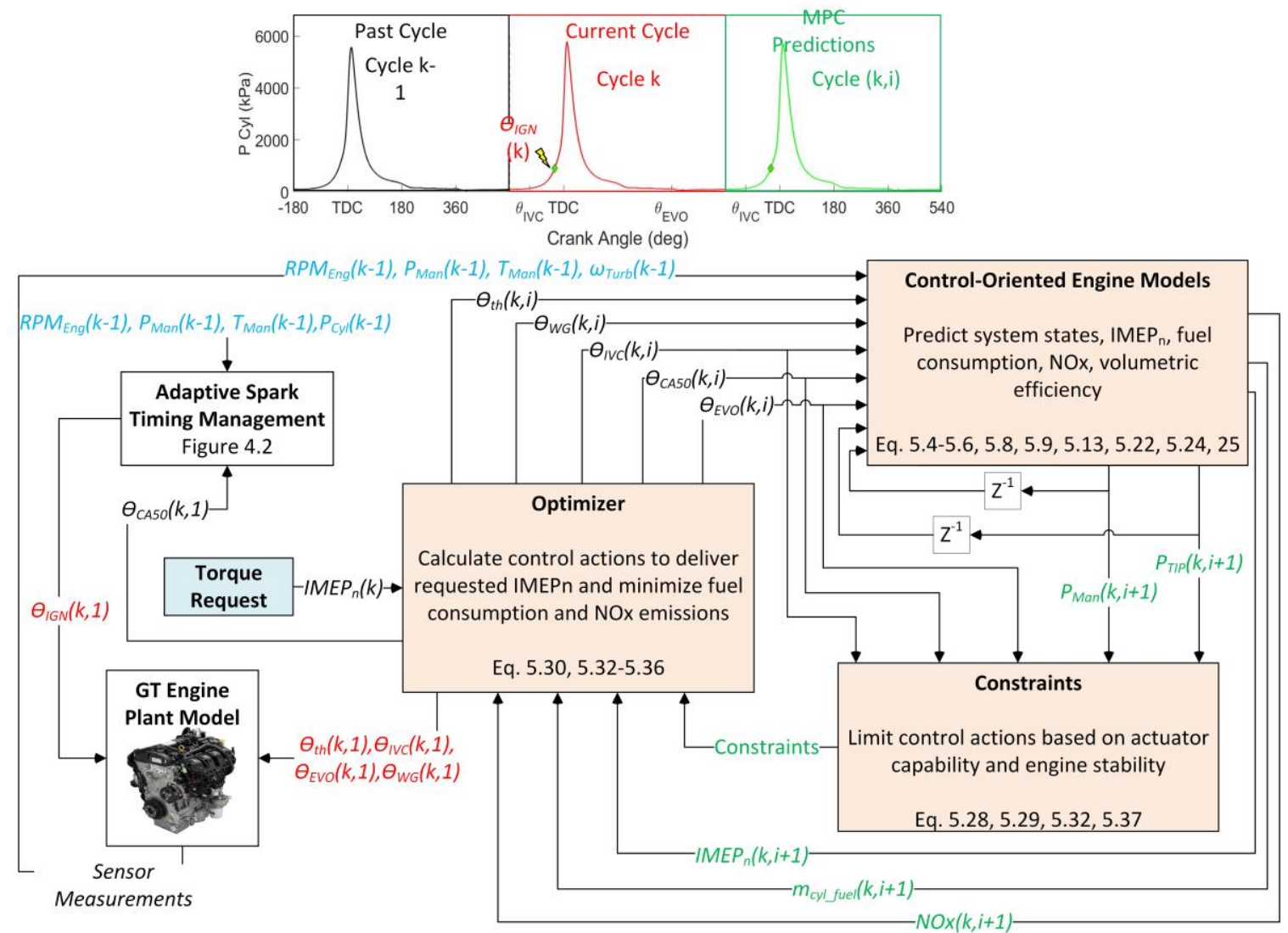

Figure 5.2 Model-based Cycle-by-Cycle GTDI SI Engine Control using E-NMPC

The engine is controlled in the cycle domain. Cycle ' $k-1$ ' is the past cycle with production sensor measurements. Cycle ' $k$ ' is the upcoming cycle that the engine needs to be controlled to achieve the operational objectives. ' $N c$ ' is the control horizon, and the 
prediction horizon is set to be the same as the control horizon. ' $i$ ' is the cycle index within the prediction horizon. The optimizer in Figure 5.2 uses the E-NMPC to perform the online optimization, which generates a sequence of optimal control actions over the prediction horizon by minimizing the cost function with the consideration of defined constraints. Using the production sensor measurements from cycle ' $k-l$ ' and calculated control actions, Control-Oriented Engine Models are used to predict future engine behaviors. The E-NMPC calculates the sets of optimal control actions for every cycle within the prediction horizon, but only the first set of computed control actions are applied to manipulate the actuators. Then, the prediction window moves forward one cycle, and a new sequence of optimal control actions is calculated when new measurements become available.

In SI engines, ignition timing is used to control combustion phasing, which affects engine torque output, specific fuel consumption, and emissions. Combustion phasing is often defined as the crank angle of fifty percent mass fraction burned (CA50). Since the combustion process is extremely complex and CA50 is dependent on engine operating conditions, CA50 is normally modeled using nonlinear equations [47]. The adaptation technics are usually used to adapt the CA50 model to be able to cover wide engine operating conditions. To reduce the complexity and computational burden of the MPC, the optimizer directly computes CA50. The Adaptive Spark Timing Management model developed in section 4 is used to manipulate the spark timing to track the optimal CA50 command issued by E-NMPC. A recursive-least-square based adaptive combustion model is used to predict the burn duration, which is from spark timing to CA50, based on the optimal CA50 target and current engine operating conditions. The dynamic spark timing prediction algorithm uses the predicted burn duration to calculate the spark timing needed for tracking the CA50 target in the upcoming cycle.

Software-in-the-loop (SIL) simulation is an effective method of safely verifying and validating the performances of the control software for complex systems $[130,131]$ in the early development stage. SIL integrates the control system and detailed physics-based plant 
models in a virtual environment, which can significantly shorten the production development cycle and reduce the cost of rapid prototype testing. In this work, the modelbased engine control system was developed in MATLAB/SIMULINK environment. The E-NMPC algorithm and control-oriented models were implemented in SIL environment using SIMULINK S-function blocks. The Adaptive Spark Timing Management model was implemented in the MATLAB function block in Simulink environment. In the SIL simulation, a high-fidelity GT-POWER engine model was used as the plant model to provide the feedback sensor measurements to engine control system, and the engine control system applies optimal control actions to the GT-POWER engine model capable reproducing the engine physics and dynamics on a cycle-by-cycle bases.

\subsection{GT-POWER Engine Model}

A detailed 1D physics-based engine model has been built in GT-POWER, and experimental engine data were used to calibrate and validate the GT-POWER engine model.

\subsubsection{GT-POWER Engine Model Development}

Figure 5.3 shows the GT-POWER engine model development process. The complete airpath system, including intake system, exhaust system, turbocharging system, was modeled using 1D method, which provides the detailed gas dynamics, pressure wave's propagation, etc. GT-POWER flow components of both intake and exhaust systems are converted from detailed 3D CAD models of intake, cylinder head, and exhaust systems with accurate geometries using the GEM3D application. Both detailed valve lift profiles and coefficient of discharge (CD) characteristic curves of intake and exhaust valves were implemented in the GT-POWER valve sub-models. The turbocharger manufacturer's turbocharger maps were imported into the turbocharger model to predict the output power, 
mass flow rate, outlet temperatures and pressures of the turbocharging system. Combustion model is one of the most key sub-models in the engine model, which impacts the engine performance, including torque, fuel consumption, and emissions. The turbulent flame combustion model was used in this work, and 3D CAD models of the piston head and the cylinder head were used to simulate the flame propagation in the engine. Since the heat transfer between in-cylinder mixture and the cylinder wall affects the combustion process and engine performance, such as thermal efficiency, IMEPn, CA50, the heat transfer model developed in [47] was used with the consideration of detailed in-cylinder flow. For predicting NOx emissions, the extended Zeldovich mechanism was used to model the total NOx produced in the cylinder at exhaust valve opening phase.

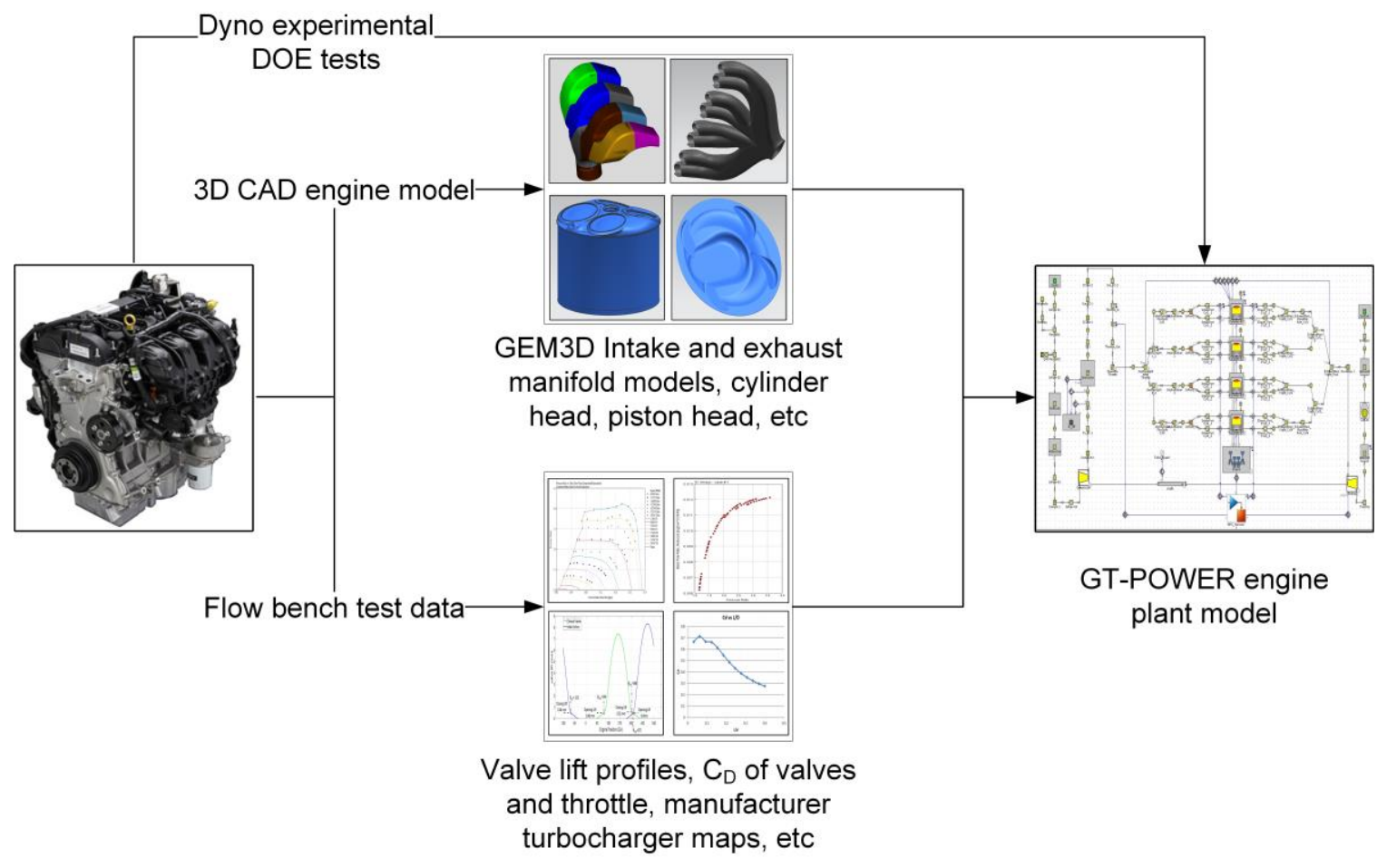

Figure 5.3 GT-POWER Engine Model Development 


\subsubsection{Calibration and Validation of the GT-POWER Engine Model}

To calibrate the GT-POWER engine model, experimental data measured from steady-state dyno tests were used. Table 3.1 lists the operating points of dyno tests. There are two steps in the GT-POWER model calibration process. First, three pressure analysis (TPA) method, which uses experimental crank angle based traces of MAP, in-cylinder pressure, and exhaust manifold pressure, was used to calibrate the overall in-cylinder heat transfer coefficient by matching simulated in-cylinder pressure traces and air flow into the cylinder to experimental data. In this step, in-cylinder conditions at IVC, such as volumetric efficiency, residual gas fraction (RGF), in-cylinder temperature of mixtures and air mass at IVC, were calculated by TPA.

In the second step, the predictive turbulent SI combustion and NOx submodels were calibrated using closed volume pressure analysis and in-cylinder quantities calculated from TPA. Table 5.1 lists the tunable model calibration parameters of two models with optimized values. The 'design optimizer' toolbox was used to derive best calibration parameters of both models over the entire experimental tests listed in Table 3.1. The genetic algorithm (GA) was used for the optimization with the parameters listed in Table 5.2. Only one set of model parameters was derived for each model to cover engine operating range. For the combustion model, the parameters were calibrated by minimizing the root-meansquared errors (RMSE) between the simulated burn rate and experimental burn rate in the crank angle domain. For the NOx model, the parameters were calibrated by minimizing the deviations between simulated cumulative NOx at EVO and experimental data. Figure 5.4 gives the key calibration results of the combustion model at early flame development stage (period between spark timing location and crank angle of 10\% fuel burned, B0010), CA50, IMEPn, and NOx model at EVO. Correlation coefficients between simulated and experimental results are all larger than 0.96. The GT-POWER model is a mean value model, which is not able to simulate cycle-by-cycle combustion variations. 
Table 5.1 Model Calibration Multipliers in GT-POWER

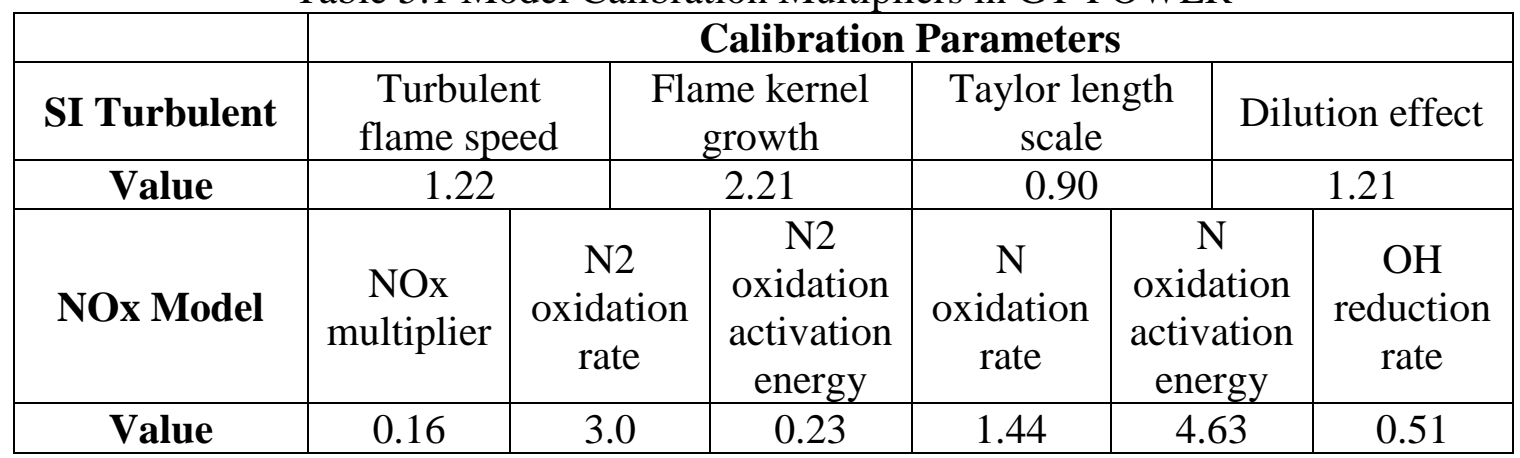

Table 5.2 Genetic Algorithm Settings

\begin{tabular}{|c|c|c|c|c|}
\hline Parameter & $\begin{array}{c}\text { Population } \\
\text { size }\end{array}$ & $\begin{array}{c}\text { Number of } \\
\text { generations }\end{array}$ & Mutation rate & $\begin{array}{c}\text { Crossover } \\
\text { rate }\end{array}$ \\
\hline Value & 30 & 34 & 0.5 & 1 \\
\hline
\end{tabular}
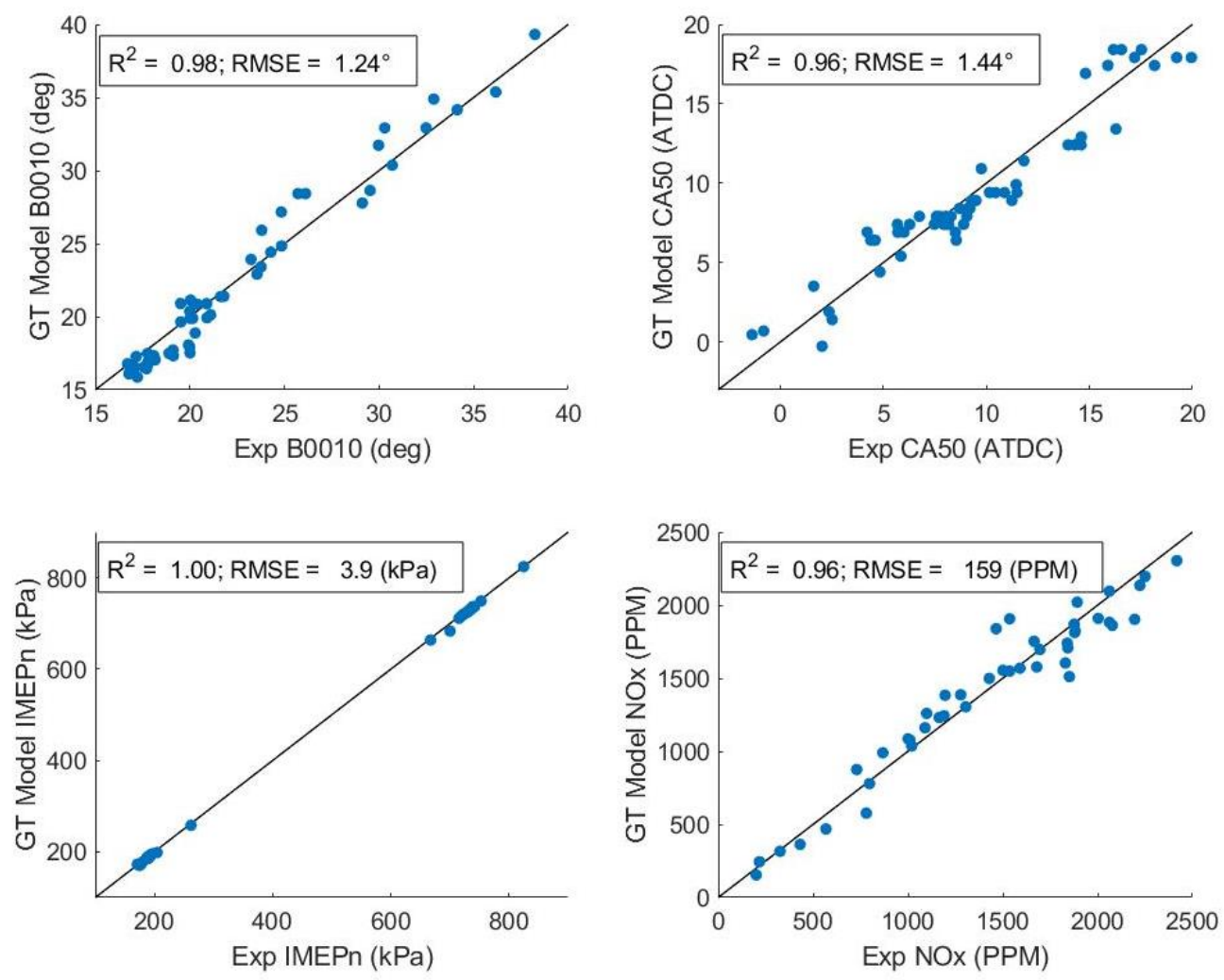

Figure 5.4 GT-POWER Combustion Model and NOx model Calibration Results 
Figure 5.5 is the complete GT-POWER engine model with the SIMULINK harness. For each upcoming engine cycle, E-NMPC based engine control system built in the SIMULINK environment receives the needed measurements from the GT-POWER engine model. Then, the E-NMPC sends the computed optimal combination of throttle angle, spark timing, intake and exhaust cam phasing, and wastegate opening percentage to the GT-POWER model through the SIMULINK harness

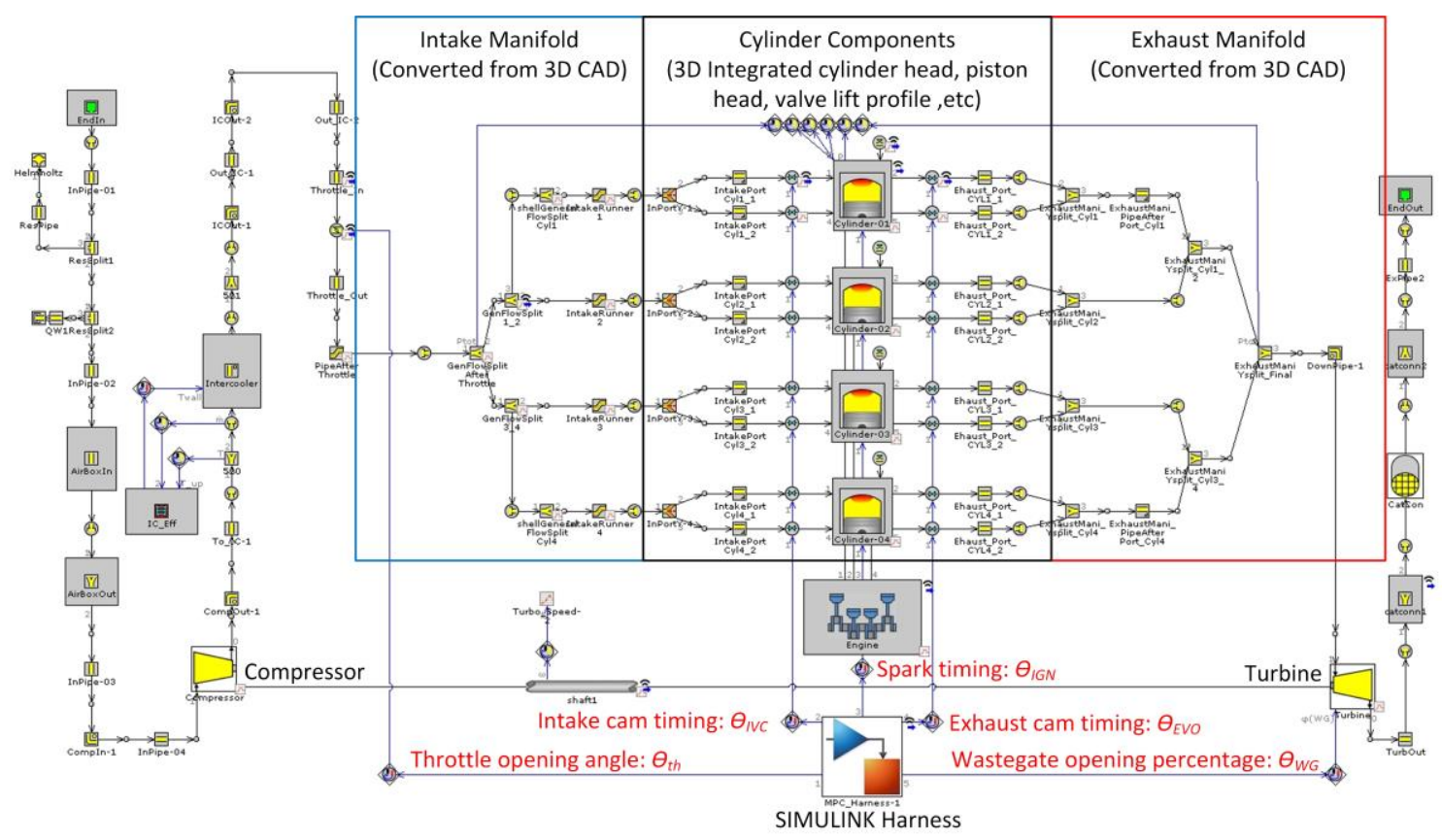

Figure 5.5 GT-POWER Engine Model

To validate the GT-POWER engine model, a transient test conducted in the test cell was used to determine the transient performance. To check the predictive performances of the engine plant model, the operating points in the first transient test were not included in the calibration data sets shown in Table 3.1. Experimental data including engine speed, ambient temperature, etc. and the engine control input for spark timing, intake valve cam timing, exhaust valve timing, were used as the inputs to the GT-POWER engine model. Figure 5.6 shows the validation results. Experimental data of CA50 and IMEP has cycleby-cycle combustion variations. Since the GT-POWER model is a mean value model, to examine the simulated CA50, the filtered CA50 was acquired by using the MATLAB function 'filtfilt', which performs the zero-phase filtering. The average model errors of 
MAP, CA50, IMEPn, and gross IMEP are $-0.03 \mathrm{kPa},-0.7 \mathrm{deg}, 5.1 \mathrm{kPa}$, and $2.2 \mathrm{kPa}$, respectively. The transient performances of the GT engine model match well with the experimental data, which indicates that the developed GT-POWER engine model well captures nonlinear dynamics and is ready to be used as a plant model for the control system development and validation.
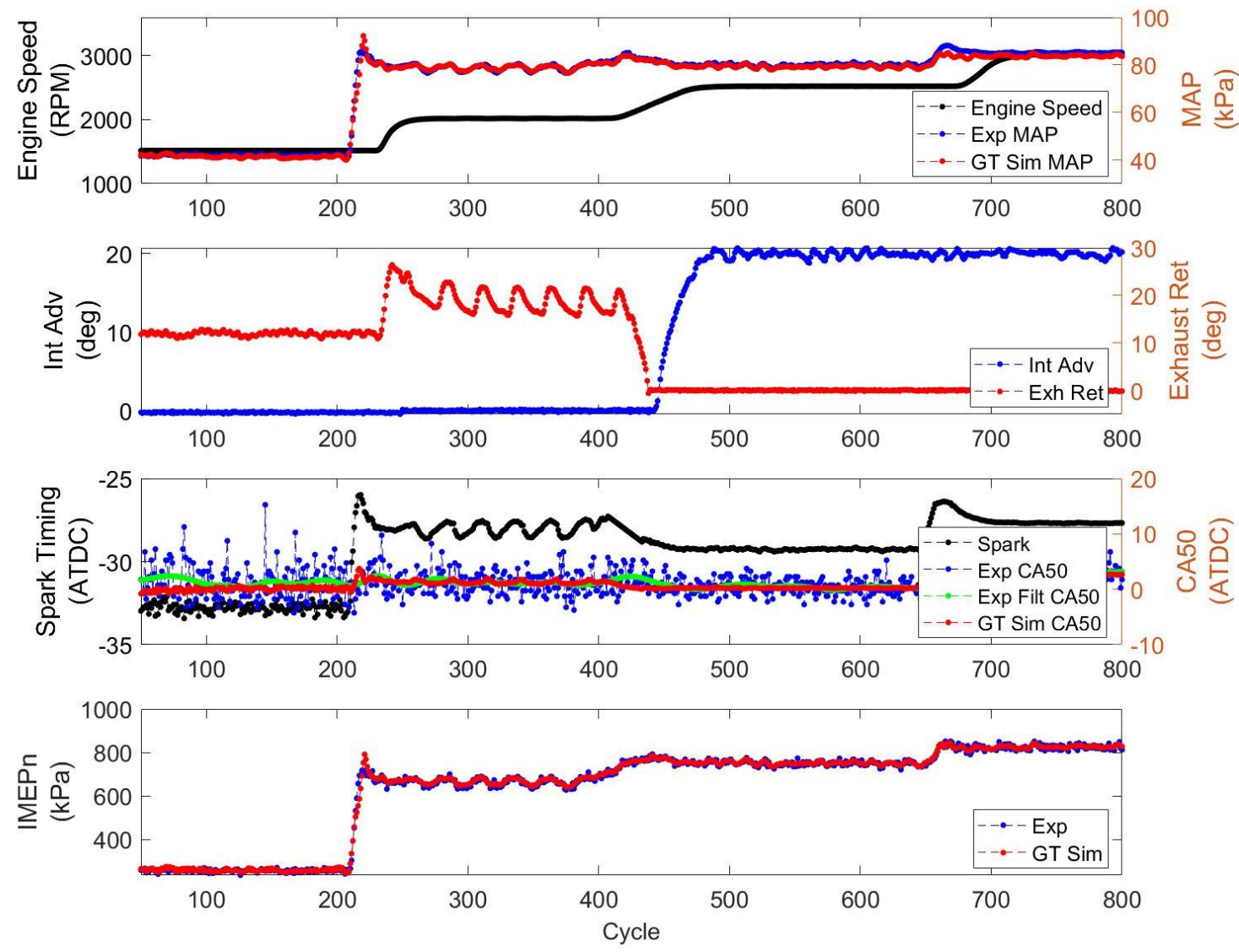

Figure 5.6 GT-POWER Engine Model Transient Validation

\subsection{Control-Oriented Engine Models}

The completed set of control-oriented GTDI SI engine model consists of airpath models and in-cylinder models. As previously discussed, both physics-based and data-drive based modeling approaches have been utilized to obtain effective and computationally efficient models for real-time E-NMPC based engine control system. The control-oriented engine 
models are calibrated using steady-state GT-POWER DOE simulations, and the operating points of DOE simulation are shown in Table 5.3.

Table 5.3 GT-POWER Steady State DOE Simulation for Control-Oriented Engine Model Development

\begin{tabular}{|c|c|c|c|c|c|c|}
\hline $\begin{array}{c}\text { Controlled } \\
\text { Parameters }\end{array}$ & $\begin{array}{c}\text { Engine } \\
\text { Speed } \\
(\mathbf{r p m})\end{array}$ & $\begin{array}{c}\text { IMEPn } \\
\text { (bar) }\end{array}$ & $\begin{array}{c}\text { CA50 } \\
\text { (deg } \\
\text { ATDC) }\end{array}$ & $\begin{array}{c}\text { Intake } \\
\text { Adv } \\
\text { (deg) }\end{array}$ & $\begin{array}{c}\text { Exhaust } \\
\text { Ret } \\
(\mathbf{d e g})\end{array}$ & $\begin{array}{c}\text { Wastegate } \\
\text { Opening } \\
(\%)\end{array}$ \\
\hline \multirow{2}{*}{ Value } & $\begin{array}{c}1500, \\
2500,\end{array}$ & $\begin{array}{c}2.5,3.0, \\
\ldots, 10.0,10.5\end{array}$ & 8,18 & $0,25,50$ & $0,25,50$ & $0,50,100$ \\
\hline
\end{tabular}

\subsubsection{Intake manifold dynamics model}

For the GTDI SI engine, torque delivery is controlled by preparing accurate amounts of air and fuel in the cylinder and igniting in-cylinder mixture at the appropriate crank position. The fuel injection system maintains the stoichiometric AFR (14.07) to maximize the efficiency of three-way-catalyst. To accurately and responsively deliver requested torque, the actuators in the airpath need to be manipulated such that the correct amount of fresh air is trapped in the cylinder at IVC. Considering the engine as a pump that inducts the air that flows through the throttle, the intake manifold, and intake valves, trapping the target amount of fresh air in the cylinder at IVC means providing corresponding boost pressure and throttle according to the needed MAP. Thus, correctly modeling the intake manifold dynamics and the boost pressure dynamics are two key aspects of estimating the fresh air mass trapped at IVC for torque delivery.

For modeling the intake manifold dynamics, the mass balance and ideal gas law were used, as described in equation (5.2) and (5.3), 


$$
\begin{gathered}
\dot{m}_{\text {th_air }}-n_{c} \cdot \dot{m}_{c y l \_a i r}=\dot{m}_{\text {Man_air }} \\
\dot{m}_{\text {Man_air }}=\frac{V_{\text {Man }}}{R \cdot T_{M a n}} \cdot \dot{P}_{\text {Man }}
\end{gathered}
$$

Where,

$$
\begin{array}{ll}
\dot{m}_{\text {th_air }} & \text { Air mass flow through the throttle }(\mathrm{g} / \mathrm{s}) \\
\dot{m}_{c y l \_a i r} & \text { Air mass flow into the cylinder }(\mathrm{g} / \mathrm{s}) \\
\dot{m}_{\text {Man_air }} & \text { Air mass flow through the intake manifold }(\mathrm{g} / \mathrm{s}) \\
V_{\text {Man }} & \text { Manifold volume }\left(\mathrm{m}^{3}\right) \\
\mathrm{R} & \text { Gas constant for dry air, } 287 \mathrm{~J} / \mathrm{kg} \cdot \mathrm{K}
\end{array}
$$

The fresh air mass flow through the throttle can be modeled as an ideal gas with constant specific heats flows through the orifice [26] as shown in equation (5.4). For an unchoked flow, when $P_{\text {Man }} / T_{\text {Man }}$ is larger than 0.528, air mass flow through the throttle is dependent on boost pressure, intake manifold pressure, and the effective opening area of the throttle. For a choked flow, $\psi\left(P_{\text {Man }} / T_{\text {Man }}\right)$ equals to 0.6847 , and airflow through the throttle only depends on $P_{T I P}, C_{d_{-} t h}$, and $A_{t h}$. Since both $C_{d_{-} t h}$ and $A_{t h}$ depends on throttle crosssectional open area, $C_{d_{-} t h}$ and $A_{t h}$ can be lumped together to form an integrated term expressing the cross-sectional opening area of the throttle, and there are many validated models for the lumped $C_{d_{-} t h}\left(\theta_{t h}\right) \cdot A_{t h}\left(\theta_{t h}\right)$ term [26, 97]. Equation (5.6) describes the lumped term used in this study. Model coefficients, $a_{1}$ to $a_{4}$, were calibrated to match modeled fresh air flow through throttle with experimental data. The model coefficients $a_{1}$ to $a_{4}$ are determined by constrained nonlinear optimization method using MATLAB function 'fmincon' to minimize equation (5.7). 


$$
\begin{aligned}
& \dot{m}_{t h \_a i r}=C_{D_{-} t h}\left(\theta_{t h}\right) \cdot A_{t h}\left(\theta_{t h}\right) \cdot \frac{P_{T I P}}{\sqrt{R \cdot T_{T I P}}} \cdot \Psi\left(\frac{P_{M a n}}{P_{T I P}}\right)
\end{aligned}
$$

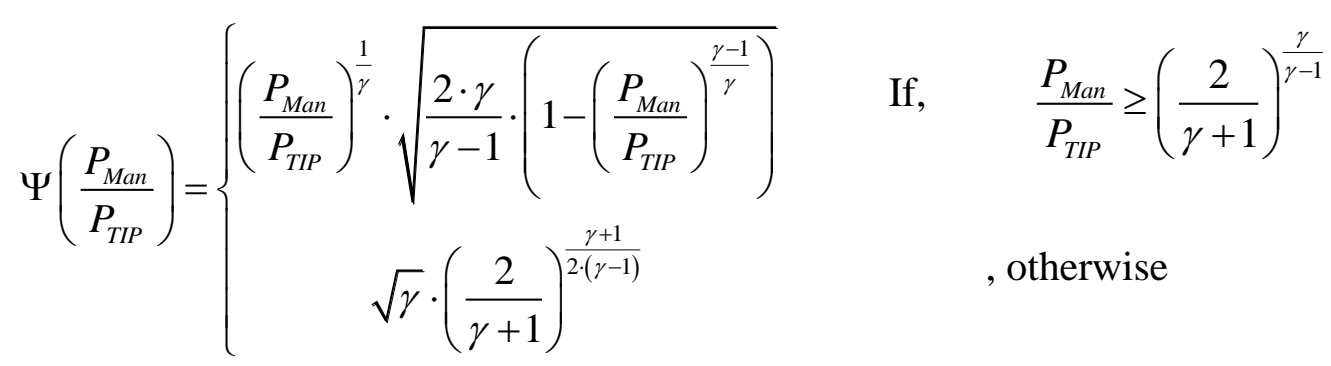

$$
\begin{aligned}
& C_{D_{-} t h}\left(\theta_{t h}\right) \cdot A_{t h}\left(\theta_{t h}\right)=a_{1} \cdot\left(1-\cos \left(a_{2} \cdot\left(\theta_{t h}\right)-a_{3}\right)\right)+a_{4} \\
& R M S E_{t h \_a i r}=\sqrt{\frac{\sum_{m=1}^{n}\left(\dot{m}_{t h_{-} a i r_{-} i n}(m)-\dot{m}_{t h_{-} a i r_{-} \exp }(m)\right)^{2}}{n-1}}
\end{aligned}
$$

Where,

$$
\begin{array}{ll}
C_{d_{-} t h} & \text { Discharge coefficients of the throttle body } \\
A_{t h} & \text { Effective throttle cross-sectional opening area }\left(\mathrm{m}^{2}\right) \\
T_{T I P d_{-} t h} & \text { Throttle inlet temperature }(\mathrm{K}) \\
\Upsilon & \text { Specific heat capacity ratio, } 1.4 \text { for dry air } \\
\dot{m}_{t h \_a i r \_e x p} & \text { Experimental air mass flow through the throttle }(\mathrm{g} / \mathrm{s})
\end{array}
$$

Estimating the mass of fresh air inducted into the cylinder is important for torque delivery and fueling control. For modeling the fresh air mass flow into the cylinder, the speeddensity method has been widely used, as shown in equation (5.8). Since the components in the intake airpath restrict the amounts of air that can be inducted in the cylinder, the volumetric efficiency $\left(\eta_{v}\right)$ is used to quantify the effectiveness of the air induction process [26]. 


$$
\dot{m}_{c y l_{\text {a }} a i r}=\eta_{v} \cdot \frac{R P M_{E n g} \cdot V_{d}}{120 \cdot T_{\text {man }} \cdot R} \cdot P_{\text {man }}
$$

Where,

$$
\begin{array}{ll}
\eta_{v} & \text { Volumetric efficiency }(\%) \\
V_{d} & \text { Cylinder displacement volume }\left(\mathrm{m}^{3}\right)
\end{array}
$$

Substituting equations (5.3) to (5.6) and (5.8) into equation (5.2), equation (5.9) gives the model of the intake manifold dynamic, which is a first order nonlinear differential equation.

$$
\begin{aligned}
& \frac{d P_{\text {Man }}}{d t}=-\eta_{v} \cdot \frac{n_{c} \cdot R P M_{E n g} \cdot V_{d}}{120 \cdot V_{\text {Man }}} \cdot P_{\text {man }} \\
& +\frac{R \cdot T_{\text {Man }}}{V_{\text {Man }}} \cdot\left(C_{D_{-} t h}\left(\theta_{t h}\right) \cdot A_{t h}\left(\theta_{t h}\right) \cdot \frac{P_{T I P}}{\sqrt{R \cdot T_{T I P}}} \cdot \Psi\left(\frac{P_{M a n}}{P_{T I P}}\right)\right)
\end{aligned}
$$

\subsubsection{Neural Network based Models}

The neural network is a popular system identification method that can capture nonlinear dynamics of MIMO systems, and mathematical equations can be derived from measured input and output signals to predict system behaviors. Different types of neural networks are being applied in the automotive industry [132], including feedforward neural networks (NNs), recurrent neural networks (RNNs), convolutional neural networks (CNNs), etc. For real-time implementation, the computational burden is related to the number of arithmetic operations used to compute the output of the model. Among various NNs, feedforward neural networks have the simplest architectures, which are computationally efficient and easy to be linearized. The feedforward NNs have been widely used for developing system models in MPC based engine control [103, 107, 133, 134]. In this study, the feedforward 
NNs were used to estimate volumetric efficiency, throttle inlet pressure, IMEPn, NOx, Coefficient of variation of IMEP (COVIMEP) and knock intensity.

Figure 5.7 shows the architecture of a typical multilayer feedforward NN. ' $m$ ' is the index of the hidden layer. ' $n$ ' is the index of the neuron numbers in each hidden layer. ' $\mathrm{W}_{\mathrm{m}, \mathrm{n}}$ ' and ' $b_{m, n}$ ' are weights and bias for each neuron. ' $f_{n m}$ ' represents the neuron with the activation function, as described in equation (5.10). Equation (5.11) is the summed input of each neuron. Considering the capabilities of capturing nonlinear dynamics and being easy to be linearized in the NMPC algorithm, the 'logsig' function was used as the activation function in all neurons in this work, as shown in equation (5.10). To prevent the activation function from being saturated, the 'preprocessing' block was used to normalize all inputs in the range of -1 and 1 . The 'postprocessing' block after the output layer was used to convert normalized values to the values with the engineering units.

$$
\begin{gathered}
f_{m n}(X)=1 /(1+\exp (-X)) \\
X=b_{m, n}+\sum_{j=1}^{n} W_{m, j} \cdot x_{j}
\end{gathered}
$$

Where,

$x_{j} \quad$ Inputs of each neuron 


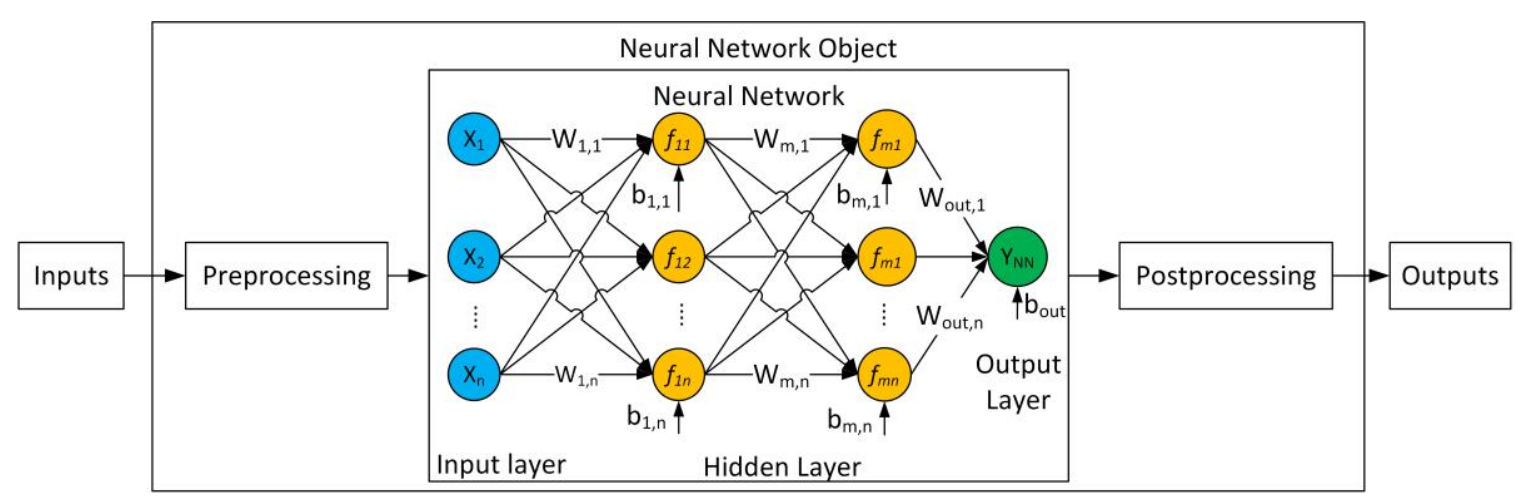

Figure 5.7 Feedforward Neural Network Architecture

During the NN development period, determining the NN's layer number and the neuron number in each layer is a important step. As previously discussed, the computational burden is related to the number of arithmetic operations. For the purpose of the real-time implementation of the NN-based model, the objective is to find a $\mathrm{NN}$ with the minimum size that can provide the acceptable model accuracy. There are two statistical terms used to quantify the performances of the NN models. The first measure is the 'bias', which is the error between the known outputs of the training data set and the predictions of the NN using the inputs from the training data set. The second measure is 'variance', which is the difference between known targets of the validation data set and the outputs of the NN using the inputs from the validation data set. The optimal NN should ensure that both the bias and variance errors are within the acceptable error ranges. The common methods used for reducing the bias error are increasing the numbers of the neural and layers of the NN. However, increasing the size of the NN may lead to higher variance errors, which is known as the overfitting problem. To avoid the overfitting issue, the Bayesian regularization [135, 136] method was used to train the feedforward NNs in this work. The feedforward NNs were trained using MATLAB, and the Bayesian regularization backpropagation method was used as the train function. Equation (5.12) is the lost function that needs to be minimized in the Bayesian regularization backpropagation, and it helps to prevent the weights of some neurons from being too large or too small by including a squared weights penalty. 


$$
L_{b}=\alpha \cdot \sum_{i=1}^{N}\left(Y_{N N}(i)-Y_{E x p}(i)\right)^{2}+\beta \cdot W^{T} \cdot W
$$

Where,
$N$
Number of training data points
$Y_{N N}$
$\mathrm{NN}$ outputs for inputs from the training data set
$Y_{\operatorname{Exp}}$
Experimental target data
$\alpha$ and $\beta$
Hyperparameters in the Bayesian regularization

Normally, the trial and error method is used to evaluate various configurations. However, this process is time consuming. This work proposes an auto-optimization method used for finding the best architecture of the feedforward NN for each data-driven based engine model, as shown in Figure 5.8. The experimental data used for developing the NN models were separated into two parts. $70 \%$ of the experimental data forms the training set, and the remaining $30 \%$ is the validation set. In this work, the Bayesian optimization was used to find optimal values of layer number and the number of hidden neurons in each layer for the feedforward NNs, which intends to minimize the RMSE between the NN validation results and the actual outputs of the validation date set. The neuron number was set to be within a range between 1 and 25 for each layer. It was found that 1-layer or 2-layer NNs are enough for control-oriented engine models, and the maximum layer number was set to be 2. At the end of the optimization process, the RMSE plot of validation data set was created, which specified the neuron number of each layer as the independent parameters, and RMSE was set to be the response parameter. Based on the RMSE plots, the NN with the minimum size and acceptable RMSE was selected for corresponding engine models. 


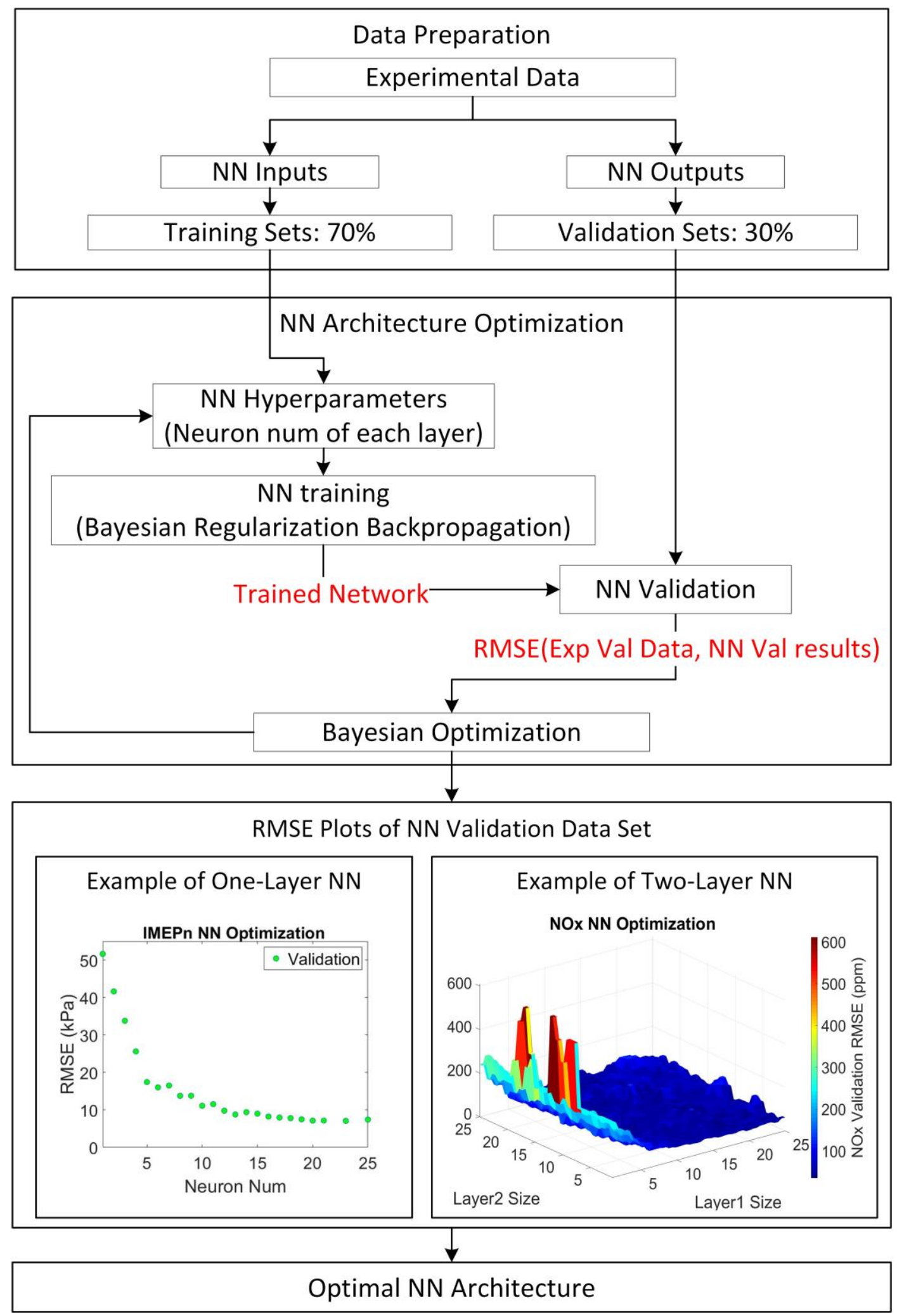

Figure 5.8 Feedforward Neural Network Architecture Optimization 


\subsubsection{Volumetric Efficiency Model}

In a production engine calibration, the volumetric efficiency is normally determined from numerous experimental tests and implemented as look-up tables [137], which can be dependent on engine speed, MAP, intake manifold temperature, etc. Since on-road SI engines are always under transient operating conditions, interpolating or surface fitting methods are normally used to estimate $\eta_{v}$ from multi-input lookup tables, and the accuracies and stabilities of $\eta_{v}$ estimation are normally impacted by interpolating and fitting methods [138]. To overcome above issues, many researchers investigated the neural network based VE models [139-141], which take engine speed, MAP, intake and exhaust cam timings as inputs, considering the engines equipped with VVT. In [142], a volumetric efficiency model was developed using the energy balance, which includes the effect of the exhaust manifold pressure on gas exchanges process during the intake stroke. Equation (5.13) describes the input-output (I/O) mapping of the VE, and the neural network was used to predict VE. For the turbocharged engine, the wastegate opening percentage is included to consider the effect of exhaust pressure on VE.

$$
\eta_{v}=f_{V E}\left(R P M_{E n g}, P_{M a n}, \theta_{I V C}, \theta_{E V O}, \theta_{w g}\right)
$$

\subsubsection{Throttle inlet pressure model}

As shown in equation (5.9), $P_{T I P}$ is one of the key parameters that impacts the intake manifold dynamics. The inlet air is compressed by the turbocharger compressor, which is connected to the turbine through the turboshaft. The energy of the exhaust gas stream is used to power the turbine. An electrical wastegate is used to regulate the amount of the exhaust gases flowing into the turbine, thus control the shaft rotating speed and the boost pressure level. Because of the mass moment of inertial of the turbine, the time is needed for the exhaust gases to spin the turbine to a certain speed and generate the desired boost pressure. This response delay is inevitable and normally called turbo lag, which describes 
the time required to reach target engine power output in response to a throttle change. To precisely estimate the throttle inlet pressure, many researchers have investigated the physics based turbocharger models $[143,144]$ by incorporating manufacturer operating maps of the turbine and the compressor. The schematic of the turbocharger equipped on the test engine is shown in Figure 5.1. Table 5.4 lists the ordinates for the turbocharger maps.

Table 5.4 Ordinates for Turbocharger Maps

\begin{tabular}{|c|c|c|c|c|}
\hline $\begin{array}{c}\text { Mechanical } \\
\text { Component }\end{array}$ & \multicolumn{2}{|c|}{ Compressor } & \multicolumn{2}{c|}{ Turbine } \\
\hline Map Output & $\dot{m}_{c}$ & $\eta_{c}$ & $\dot{m}_{t}$ & $\eta_{t}$ \\
\hline Input & $P_{c_{\_} \text {out }} / P_{c_{\_} \text {in }}, \omega_{\text {turb }}$ & $P_{t_{\_} \text {out }} / P_{t_{\_} \text {in }}, \omega_{\text {turb }}$ \\
\hline
\end{tabular}

Where,

$\begin{array}{ll}P_{c_{-} \text {out }} & \text { Compressor outlet pressure }(\mathrm{Pa}) \\ P_{c_{-} \text {in }} & \text { Compressor outlet pressure }(\mathrm{Pa}) \\ P_{t_{-} \text {in }} & \text { Turbo inlet pressure }(\mathrm{Pa}) \\ P_{t_{-} \text {out }} & \text { Turbo outlet pressure }(\mathrm{Pa}) \\ \eta_{c} & \text { Compressor efficiency } \\ \eta_{t} & \text { Turbine efficiency } \\ \dot{m}_{c} & \text { Air flow rate through the compressor }(\mathrm{g} / \mathrm{s}) \\ \dot{m}_{t} & \text { Air flow rate through the turbine }(\mathrm{g} / \mathrm{s})\end{array}$

For cycle-by-cycle engine control, the slow dynamics of intercooler outlet temperature is ignored, and $P_{T I P}$ is assumed to be the same as $P_{C_{-} o u t}$. Lumping parts between the compressor and the throttle, the throttle inlet pressure can be modeled using the ideal gas law and the mass balance, as described in equation (5.14). Newton's second law is used to model the dynamics of the rotating turboshaft, described in equation (5.15), where $J_{t}$ is the 
inertial of assembled parts, including the turboshaft, the turbo, and the compressor. Equations (5.16) and (5.17) are torque models of the turbine and the compressor respectively [136]. Equation (5.18) describes the dynamics of the turbine inlet pressure, and equation (5.20) calculates the total exhaust mass flow out of the cylinder. The wastegate mass flow rate is modeled using equation (5.19).

$$
\begin{gathered}
\frac{d P_{T I P}}{d t}=\frac{R \cdot T_{c_{-} \text {out }}}{V_{c_{-} \text {out } \_ \text {th }}} \cdot\left(\dot{m}_{c}-\dot{m}_{\text {th_air }}\right) \\
J_{t} \cdot \omega_{\text {turb }} \cdot \frac{d \omega_{\text {turb }}}{d t}=T_{t}-T_{c} \\
T_{t}=\dot{m}_{t} \cdot c_{p_{-} E x h} \cdot T_{t_{-} \text {in }} \cdot\left(1-\left(\frac{P_{t_{-} \text {in }}}{P_{t_{-} \text {out }}}\right)^{\frac{\gamma-1}{\gamma}}\right) \cdot \frac{\eta_{t}}{\omega_{\text {turb }}} \\
T_{c}=\dot{m}_{c} \cdot c_{p_{-} \text {Air }} \cdot T_{c_{-} \text {in }} \cdot\left(\left(\frac{P_{c_{-} \text {out }}}{P_{c_{-} \text {in }}}\right)^{\frac{\gamma-1}{\gamma}}-1\right) \cdot \frac{1}{\eta_{c} \cdot \omega_{\text {turb }}} \\
\frac{d P_{t_{\text {_in }}}}{d t}=\frac{R \cdot T_{t_{-} \text {in }}}{V_{t_{-} \text {in }}} \cdot\left(\dot{m}_{E x h}-\dot{m}_{W G}\right) \\
\dot{m}_{W G}=C_{D_{-} W G}\left(\theta_{w g}\right) \cdot A_{W G}\left(\theta_{w g}\right) \cdot \frac{P_{t_{-} \text {in }}}{\sqrt{R \cdot T_{t_{-} \text {in }}} \cdot \Psi\left(\frac{P_{t_{-} \text {out }}}{P_{t_{-} \text {in }}}\right)} \\
\dot{m}_{E x h}=n_{c} \cdot \dot{m_{c y l} \text { air }} \cdot\left(1+\frac{1}{14.07}\right) \cdot \lambda
\end{gathered}
$$

Where,

$$
\begin{array}{ll}
C_{p_{-} E x h} & \text { Specific heat of exhaust gases at constant pressure }(\mathrm{J} / \mathrm{kg} \cdot \mathrm{K}) \\
C_{p_{-} \text {Air }} & \text { Specific heat of fresh air at constant pressure }(\mathrm{J} / \mathrm{kg} \cdot \mathrm{K})
\end{array}
$$




$\begin{array}{ll}T_{t_{-} i n} & \text { Compressor outlet temperature }(\mathrm{K}) \\ \dot{m}_{E x h} & \text { Exhaust gas mass flow rate from the cylinder }(\mathrm{g} / \mathrm{s}) \\ \dot{m}_{W G} & \text { Exhaust gas mass flow rate passes the wastegate }(\mathrm{g} / \mathrm{s}) \\ V_{t_{-} i n} & \text { Total volume between the exhaust manifold and the turbine }\left(\mathrm{m}^{3}\right) \\ C_{D_{-} W G} & \text { Discharge coefficients of the wastegate }\end{array}$

Using manufacturer maps and substituting equations (5.15) to (5.20) into the equation (5.14), gives the physics-based turbocharger model, where the throttle inlet pressure can be estimated using the equation (5.21), where $\eta_{c}, \eta_{t}, P_{c_{-} \text {in }}, P_{c_{-} \text {out }}, P_{t_{-} \text {in }}, P_{t_{-} \text {out }}, T_{c_{-} \text {in }}$, $T_{c_{-} \text {out }}$, and $T_{t_{-} \text {in }}$ are all intermediate parameters. Since the turbocharger maps are discontinuous and nonlinear, directly using maps in the controller will cause discontinuity of the control system. Fitting methods, like surface fitting and polynomial fitting, are needed to represent the maps in the control algorithm. As the turbocharger model is nonlinear, fitting the nonlinear and discontinuous maps may significantly reduce the model accuracy and add additional complexities to the controller. Other parameters, like $C_{D_{-} W G}$, $A_{W G}, V_{t_{-} i n}$, and $V_{c_{-} \text {out }}$ also need to be accurately measured and calibrated. For real-time cycle-by-cycle engine control using NMPC, evaluating the above physics-based turbocharger models and taking partial derivatives of above nonlinear equations with respect to control signals in the NMPC algorithm are time consuming, and additional numerical differentiation and integration algorithms that are suitable for online implementation are required.

$$
\frac{d P_{T I P}}{d t}=f_{T I P}\left(\begin{array}{l}
R P M_{E n g}, P_{T I P}, P_{M a n}, \theta_{t h}, \theta_{I V C}, \theta_{E V O}, \theta_{w g}, \omega_{t u r b}, \eta_{c}, \eta_{t}, \\
P_{c_{-} i n}, P_{c_{-} \text {out }}, P_{t_{-} \text {in }}, P_{t_{-} \text {out }}, T_{c_{-} \text {in }}, T_{c_{-} \text {out }}, T_{t_{-} \text {in }}
\end{array}\right)
$$


For developing the control-oriented models used in the control system, system identification is widely used to derive computationally efficient mathematical models using measured input and output signals. In this study, equation (5.22) represents the throttle inlet pressure dynamics in an input-output (I/O) mapping form. The neural network was used to predict $P_{T I P}$. In this study, we assume that the measurements of the turbo speed is available.

$$
P_{T I P}=f_{T I P}\left(R P M_{E n g}, P_{M a n}, \theta_{t h}, \theta_{I V C}, \theta_{E V O}, \theta_{w g}, \omega_{t u r b}\right)
$$

\subsubsection{IMEPn Model}

In this work, IMEPn is used as the tracking reference in the E-NMPC. Equation (5.23) describes the definition of IMEPn in the thermodynamic point of view. Normally, IMEPn is calculated from measured in-cylinder pressure over the entire engine cycle $\left(720^{\circ}\right)$. As previously discussed, predicting the crank angle-based in-cylinder pressure is not applicable for real-time cycle-based engine control, so IMEPn was modeled using a feedforward $\mathrm{NN}$ in this work. In equation $(5.23), \eta_{n_{-} \text {ind }}$ is the indicated thermal conversion efficiency, which is the ratio of the actual work per engine cycle to the total chemical energy of certain amount of injected fuel released from the combustion [26]. For a specific engine with the fixed cylinder volume $\left(V_{d}\right)$ operated under stoichiometric condition, $\eta_{n_{-} \text {ind }}$ is mainly affected by MAP, exhaust pressure, CA50 [145], the effective compression ratio controlled by IVC [146], and effective expansion ratio controlled by EVO [147, 148] have high impact on IMEPn. Combining the $\eta_{v}$ modeled using Equation (5.13), IMEPn described by equation (5.23) can be modeled using equation (5.24), which is formulated in the I/O mapping form. 


$$
\begin{gathered}
I M E P_{n}=\frac{\eta_{n_{-} i n d} \cdot Q_{L H V}}{V_{d}} \cdot m_{c y l_{-} f}=\eta_{n_{-} i n d} \cdot \eta_{v} \cdot \frac{R P M_{E n g} \cdot P_{M a n} \cdot Q_{L H V}}{120 \cdot T_{M a n} \cdot V_{d} \cdot A F R_{S t o i}} \\
I M E P_{n}=f_{I M E P_{n}}\left(R P M_{E n g}, P_{M a n}, T_{M a n}, \theta_{I V C}, \theta_{C A 50}, \theta_{E V O}, \theta_{W G}\right)
\end{gathered}
$$

Where,

$$
\begin{array}{ll}
\eta_{n_{-} \text {ind }} & \text { net indicated thermal conversion efficiency } \\
Q_{L H V} & \text { Lower heating value of E10, } 41282 \mathrm{KJ} / \mathrm{Kg} \\
A F R_{\text {Stoi }} & 14.07 \text { for E10 }
\end{array}
$$

\subsubsection{NOx Model}

One of the objectives of the presented engine control system is minimizing engine out NOx emissions. In this work, NOx is measured by a Cambustion fast gas analyzer in the unit of parts per million (ppm). For an SI engine operated under the stoichiometric condition, thermal mechanism is the dominant factor that leads to NOx formation, and the reactions of $\mathrm{N}$ and $\mathrm{O}_{2}$ take place in the high temperature burned gas region. Although the physicsbased NOx model described by the extended Zeldovich mechanism includes detailed chemical reactions, the requirements of the precise modeling of in-cylinder chemical reactions and high computation effort make it not suitable for the online model-based NOx control application. The formation of $\mathrm{NOx}$ is sensitive to the maximum cylinder temperature, residual burned gas fraction (RGF) of the gas mixture, and spark timing [26]. The total residual gas trapped at IVC comprises two parts. The first part is burned gas remained in the cylinder after the exhaust process, which is trapped at IVO. The second part is exhaust gas re-breathed from the exhaust manifold into the cylinder during the positive valve overlap period [26]. The total RGF is governed by the relative pressures of MAP, in-cylinder pressure, and exhaust manifold during the gas exchange phase, which is controlled by intake and exhaust cam timings and engine speed. The exhaust pressure is affected by the wastegate opening percentage. Both the RGF and the combustion phasing impact the maximum in-cylinder temperature. Based on the above discussions, a 
feedforward NN was used to predict the total NOx formation at EVO, as described in equation (5.25).

$$
N O x=f_{N O x}\left(R P M_{E n g}, P_{M a n}, T_{M a n}, \theta_{I V C}, \theta_{C A 50}, \theta_{E V O}, \theta_{W G}\right)
$$

\subsubsection{Knock Model}

Engine knock is caused by self-ignition of the in-cylinder mixtures ahead of the flame front, which results in an extremely rapid release of the chemical energy and results in high local pressures in the combustion chamber [26]. Engine knock is an abnormal combustion, and severe engine knock will damage the engine. In the production engines, the knock sensors are used detect the know events. Both map-based feedforward controller and closed-loop feedback controller are used to suppress the knock. In the E-NMPC based engine control system, a predictive knock model is crucial for avoiding engine knock and safely operating the engine within the limits. Knock intensity is one of the popular knock metrics used to correlate to the significance level of the engine knock. Equation (5.26) shows the knock intensity calculation using the in-cylinder pressure signals [149], and both the engine speed and in-cylinder pressure determine the calculation of $K I_{\text {Exp }}$. Where, $P_{f}$ is the filtered incylinder pressure, $\theta$ is the crank angle, and $\mathrm{t}$ is the time for the corresponding crank angle. As previously discussed, instead of predicting the in-cylinder pressure for knock prediction, a NN-based control-oriented knock model was developed and used in the feedforward loop to suppress the knock in the upcoming engine cycle, using available control signals and production sensor measurements. Various experimental validated empirical correlations have been developed to predict the knock intensity, and equation (5.27) shows the general form of KI correlation models [150], where $\mathrm{T}$ and $\mathrm{P}$ are temperature and pressure. In this work, there was no EGR. Instead, the residual gas fraction controlled by VVT is considered in this work. In equation (5.27), the pressure and temperature of the end gas are the key parameters for correlating the knock intensity, which

are affected by MAP, IVC, CA50, and $T_{\text {Man }}$. Thus, equation (5.28) is the control-oriented KI correlation model used for knock prediction in the future cycles. Due to the confidential 
contents of the knock test data, the experimental KI values were normalized into a specific range. Normalized KI values were used to train and validate NN-based KI model.

$$
\begin{aligned}
& K I_{\text {Exp }}=\frac{1}{t_{2}-t_{1}} \cdot \int_{t_{1}\left(\theta_{1}\right)}^{t_{2}\left(\theta_{2}\right)} P_{f}(t) d t \\
& K I_{\text {corr }}=c_{1} \cdot T_{\text {end } c_{-} \text {gas }}^{c_{2}} \cdot P_{\text {end } \_ \text {gas }}^{c_{3}} \cdot \lambda^{c_{4}} \cdot e^{\frac{c_{5}}{T_{\text {end }} \text { gas }}} \cdot\left(1-c_{6} \cdot E G R\right)^{c_{7}} \\
& K I=f_{K I}\left(R P M_{E n g}, P_{M a n}, T_{M a n}, \theta_{I V C}, \theta_{C A 50}, \theta_{E V O}\right)
\end{aligned}
$$

Where,

$$
\begin{array}{ll}
c_{1} \text { to } c_{7} & \text { Model coefficients } \\
E G R & \text { Exhaust gas recirculation }
\end{array}
$$

\subsubsection{COV of IMEP Model}

Cycle-to-cycle combustion variations (CCVs) are natural characteristics of all SI engines, which are normally described by cyclic fluctuations of IMEP. Cyclic combustion variations have been studied through measurements of in-cylinder pressure traces using in-cylinder pressure sensors and visualization of cycle-by-cycle in-cylinder flame propagation via flow field measurement by particle imaging velocimetry $[32,151]$. There are three main factors resulting in $\mathrm{CCVs}$, variation in in-cylinder flow motions, variation in mass of fuel and air in each cycle, and variation in mixture composition in the cylinder and near spark plug [26]. All above three factors are impacted by engine operating conditions, which results in different levels of CCVs. Since higher cyclic combustion variations may lead to higher engine vibrations and worse drivability, which can be perceptible to the driver, the manufactures set the threshold of CCVs to ensure the drivability and comfort. COVIMEP is commonly used to indicate the levels of CCVs. Many researchers developed different types of models to correlate the COVIMEP with engine operating conditions, including 
neural network based models [152, 153], polynomial regression models [154, 155], etc. Based on the available sensors and control signals, a control-oriented COVIMEP model was built using a NN. In modern engines, VVT is widely used to introduce residual gases into the cylinder, which helps to improve the fuel economy and decrease the emissions. However, more RGF results in longer burn durations, which leads to higher CCVs. In this study, engine speed, MAP, and cam timings were included to consider the effects of the in-cylinder flow motions. CA50 was used to reflect the effect of combustion process on the CCVs, where combustion phasing/durations are affected by RGF. Equation (5.29) describes the I/O mapping of the developed COVIMEP model in this work. Experimental data were used to train and validate the COVIMEP model.

$$
C O V I M E P=f_{C O V}\left(R P M_{E n g}, P_{M a n}, \theta_{I V C}, \theta_{C A 50}, \theta_{E V O}\right)
$$

Table 5.5 summaries the developed control-oriented engine models. The KI model and the COVIMEP model were calibrated using experimental data. The other models were calibrated using the GT-POWER DOE tests data listed in Table 5.3.

Table 5.5 Control-Oriented Engine Models

\begin{tabular}{|c|c|c|c|c|}
\hline $\begin{array}{c}\text { Modeled } \\
\text { Parameters }\end{array}$ & \multicolumn{2}{|c|}{ Method } & $\mathbf{R 2}$ & RMSE \\
\hline$\dot{\boldsymbol{m}}_{\text {th_air }}$ & \multicolumn{2}{|c|}{$\begin{array}{l}\text { Ideal gas with constant specific } \\
\text { heats flow through the orifice }\end{array}$} & 0.99 & $0.43 \mathrm{~g} / \mathrm{s}$ \\
\hline$P_{M a n}$ & \multicolumn{2}{|c|}{$\begin{array}{l}\text { Mass conservation and ideal gas } \\
\text { law }\end{array}$} & 0.99 & $0.5 \mathrm{kPa}$ \\
\hline$P_{T I P}$ & \multirow{3}{*}{$\begin{array}{l}\text { Neural } \\
\text { Network }\end{array}$} & $\begin{array}{c}1 \text { layer, } 5 \\
\text { neurons }\end{array}$ & 0.99 & $0.18 \mathrm{kPa}$ \\
\hline$\eta_{v}$ & & $\begin{array}{c}1 \text { layer, } 19 \\
\text { neurons }\end{array}$ & 0.99 & $0.4 \%$ \\
\hline$I M E P n$ & & $\begin{array}{c}2 \text { layer, [14 4] } \\
\text { neurons }\end{array}$ & 0.99 & $6.6 \mathrm{kPa}$ \\
\hline
\end{tabular}




\begin{tabular}{|c|c|c|c|}
\hline $\operatorname{Nox}$ & $\begin{array}{c}2 \text { layer, [11 4] } \\
\text { neurons }\end{array}$ & 0.99 & 18 ppm \\
\hline COVIMEP & $\begin{array}{c}1 \text { layer, } 13 \\
\text { neurons }\end{array}$ & 0.97 & $0.3 \%$ \\
\hline$K I$ & $\begin{array}{c}2 \text { layer, [17 }\left[\begin{array}{ll}17\end{array}\right] \\
\text { neurons }\end{array}$ & 0.99 & 0.1 \\
\hline
\end{tabular}

\subsection{E-NMPC Development}

\subsubsection{E-NMPC Formulation}

Control-oriented dynamic models developed and calibrated in the previous sections are used to model the engine dynamics and predict the future engine behaviors over the prediction horizon. The engine is controlled in the cycle domain. The discrete time nonlinear state-space model described in equation (5.30) is used in E-NMPC.

$$
\left\{\begin{array}{c}
X(k+1)=f(X(k), U(k), V(k)) \\
Y(k)=f(X(k), U(k), V(k))
\end{array}\right.
$$

The state vector $\mathrm{X}$ consists of the intake manifold pressure and the throttle inlet pressure. There are five control variables in the control vector $\mathrm{U}$, throttle angle $\left(\theta_{t h}\right)$, intake cam advance angle $\left(\theta_{I V C}\right)$, combustion phasing $\left(\theta_{C A 50}\right)$, exhaust cam retard angle $\left(\theta_{E V O}\right)$, and wastegate opening percentage $\left(\theta_{W G}\right) . \mathrm{V}$ is a measured disturbance vector consisting of three online measurements, engine speed $\left(R P M_{E n g}\right)$, intake manifold temperature $\left(T_{M a n}\right)$, and turbo shaft speed $\left(\omega_{\text {turb }}\right)$. The temperature difference between throttle inlet and throttle outlet is ignored, that is $T_{T I P}$ is the same as $T_{M a n}$. Equation (5.34) are discrete state equations. The output vector consists of three outputs, including IMEPn, cylinder air mass trapped at IVC $\left(m_{c y l_{-} a i r}\right)$, and NOx emission at EVO. 


$$
\begin{aligned}
& X(k)=\left[\begin{array}{c}
P_{M a n}(k) \\
P_{T I P}(k)
\end{array}\right] \\
& U(k)=\left[\begin{array}{c}
\theta_{t h}(k) \\
\theta_{I V C}(k) \\
\theta_{C A 50}(k) \\
\theta_{E V O}(k) \\
\theta_{W G}(k)
\end{array}\right] \\
& V(k)=\left[\begin{array}{c}
R P M_{\text {Eng }}(k) \\
T_{\text {Man }}(k) \\
\omega_{\text {Turb }}(k)
\end{array}\right] \\
& X(k+1)=\left[\begin{array}{c}
P_{\text {Man }}(k+1) \\
P_{\text {TIP }}(k+1)
\end{array}\right]=\left[\begin{array}{l}
f_{\text {Man }}\left(R P M_{\text {Eng }}(k), P_{\text {Man }}(k), P_{T I P}(k), \theta_{t h}(k), \theta_{I V C}(k), \theta_{\text {EVO }}(k), \theta_{\text {wg }}(k)\right) \\
f_{\text {TIP }}\left(R P M_{\text {Eng }}(k), P_{\text {Man }}(k), \theta_{\text {th }}(k), \theta_{\text {IVC }}(k), \theta_{\text {EVO }}(k), \theta_{\text {wg }}(k), \omega_{\text {turb }}(k)\right)
\end{array}\right] \\
& =\left[\begin{array}{c}
\left.P_{\text {Man }}(k)+\left(\begin{array}{c}
-\eta_{v}(k) \cdot \frac{n_{c} \cdot R P M_{\text {Eng }}(k) \cdot V_{d}}{120 \cdot V_{\text {Man }}} \cdot P_{\text {Man }}(k)+ \\
\frac{R \cdot T_{\text {Man }}(k)}{V_{\text {Man }}} \cdot\left(C_{D_{-} \text {th }}\left(\theta_{t h}(k)\right) \cdot A_{t h}\left(\theta_{t h}(k)\right) \cdot \frac{P_{T I P}(k)}{\sqrt{R \cdot T_{T I P}(k)}} \cdot \Psi\left(\frac{P_{\text {Man }}(k)}{P_{T I P}(k)}\right)\right) \\
f_{\text {TIP }}\left(R P M_{\text {Eng }}(k), P_{\text {Man }}(k), \theta_{\text {th }}(k), \theta_{I V C}(k), \theta_{\text {EVO }}(k), \theta_{w g}(k), \omega_{\text {turb }}(k)\right)
\end{array}\right) \cdot \frac{30}{R P M_{E n g}(k)}\right)
\end{array}\right.
\end{aligned}
$$

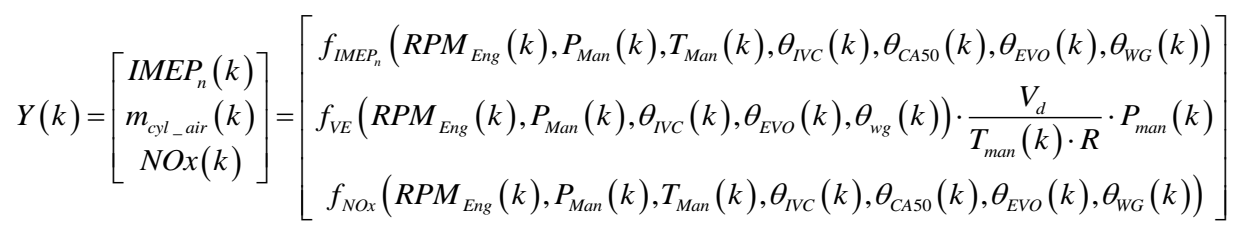

The objectives of the engine control system are to track the torque request while minimizing fuel consumption and NOx emission. An optimal control problem (OCP) has been formulated to minimize a quadratic cost function as described in equation (5.36). In the cost function, the first term is for tracking the requested IMEPn, which aims to minimize the squared error between the request IMEP ref $_{\text {and }}$ the system output IMEPn. The cycle-based fuel consumption and NOx emission over the control horizon are minimized, which are described by the second and the third term, respectively. To prevent 
unreasonable rapid changes of control actions, which may drive the system to be unstable or result in oscillated system behaviors, many researchers added constraints to limit the change rate of control signals and demonstrated the closed-loop stability of linear, discretetime systems under MPC [156]. In addition to directly imposing the constraints for change rate of control actions, a penalty term is included in the cost function to help stabilize the system. $\mathrm{A}, \mathrm{B}$, and $\mathrm{C}$ are nonnegative weighting factors. $\mathrm{D}_{1}$ to $\mathrm{D}_{5}$ nonnegative are weighting factors for change rates of control signals. These eight tunable variables need to be calibrated to satisfy the control objectives and desired system performances. Delivering the torque requests has the highest priority in the engine control system. Therefore, ' $\mathrm{A}$ ' is the dominant calibration parameter so that the torque tracking is always satisfied. Since controlling VVT to achieve fuel consumption minimization conflicts with NOx emission reduction under some engine operating conditions, ' $\mathrm{B}$ ' and ' $\mathrm{C}$ ' are balanced in a way that reducing the NOx emission within minimizing specific fuel consumption. The weighting factors, $D_{1}$ to $D_{5}$, are used to prevent aggressive control actions and ensure the smooth torque tracking performance.

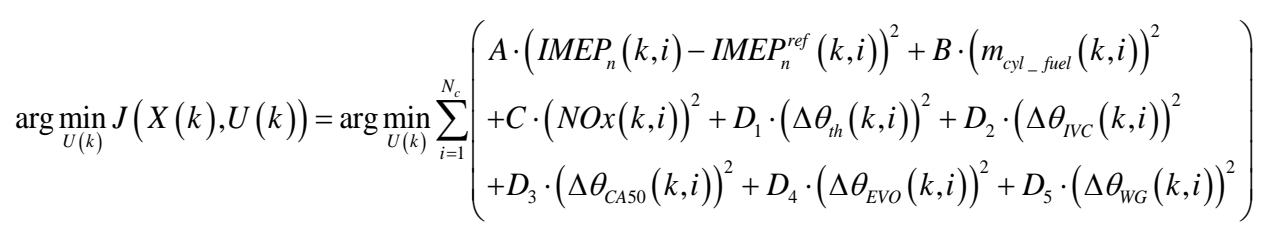

As discussed before, systematically handling the system constraints is one of the key features of MPC. The cost function in equation (5.36) is subject to constraints in equation (5.37). The first type of constraint limits the operating ranges of all mechanical actuators to ensure the physical feasibility of the system. Since the VVT actuators driven by hydraulic devices have the slowest response and time are needed to open and close the throttle and the wastegate, constraints are added to movement rate of actuators, which were determined from vehicle driving tests. COVIMEP and KI are used to avoid abnormal combustion and ensure the engine stability and avoid engine knock, respectively. Finally, 
$P_{T I P}-P_{\text {Man }}$ ensures the air flow direction and sets the upper working boundary of the turbocharger.

$$
\left\{\begin{array}{c}
0<=\theta_{t h}(k, i)<=90 \\
-25<=\Delta \theta_{t h}(k, i)<=25 \\
0<=\theta_{I V C}(k, i)<=50 \\
-20<=\Delta \theta_{I V C}(k, i)<=20 \\
0<=\theta_{E V O}(k, i)<=50 \\
-20<=\Delta \theta_{E V O}(k, i)<=20 \\
0<=\theta_{W G}(k, i)<=100 \\
-25<=\Delta \theta_{W G}(k, i)<=25 \\
5<=\theta_{C A 50}(k, i)<=20 \\
0<C O V I M E P(k, i)<=3 \\
0<=K I(k, i)<=5 \\
0<=P_{T I P}(k, i)-P_{\text {Man }}(k, i)<=100
\end{array}\right.
$$

\subsubsection{E-NMPC Implementation for SIL Co-simulation}

For cycle-by-cycle engine control, nonlinear constrained OCP in equations (5.30) to (5.37) needs to be solved once per cycle. Therefore, online algorithm with fast computational speed is crucial for real-time NMPC. This paper uses real-time iteration (RTI) based scheme developed in [157] to solve OCP, which is a sequential quadratic programming (SQP) based online solver for NMPC. For real-time implementation, the ACADO code generation tool presented in [158] and [159] was utilized to generate efficient C-code that solves the NMPC problem using SQP-based RTI algorithm. SQP is an iterative method for constrained nonlinear optimization and derives a locally optimal solution of the original OCP through applying the Newton search directions. In ACADO, an active-set QP solver, qpOASES, developed in [160] has been integrated and used to solve a sequence of QPs. Since the cost function in equation (5.36) only has least-squared terms, the generalized Gauss-Newton method is selected to approximate the Hessian matrices in QPs. To speed 
up the SQP calculations and converge to the optimal solution faster, the warm-start technique is used in the SQP. The optimal active set solution of predicted states and control actions from the previous QP is used as the initial starting values for the next QP. For the SIL co-simulation, as illustrated in Figure 5.2, a 1D high-fidelity GTDI SI engine model was built in the GT-SUITE environment, and all other models were developed in SIMULINK environment. The GT-POWER engine model receives five control signals from the SIMULINK models, and the SIMULINK models take feedback measurements from the GT-POWER engine model. The generated C-code of E-NMPC was integrated into the Simulink using an S-Function. The E-NMPC S-Function receives the cycle-based feedback measurements from the GT-POWER engine model and outputs optimal control signals to the GT-POWER engine model. Since the intake manifold dynamics model described in equation (5.9) is a first-order system, the time constant of the intake manifold system can be described by equation (5.38), which has the unit of seconds and depends on the volumetric efficiency and engine speed. Since the engine is controlled in the cycle domain, equation (5.39) converts the unit of the time constant in equation (5.38) from seconds into engine cycles. Assuming the engine speed is $3500 \mathrm{rpm}$ and $\eta_{v}$ is $50 \%$, with a step throttle change, it will take 4 cycles to reach a new intake manifold pressure. To make E-NMPC respond fast and effectively capture the intake manifold dynamics, the control horizon of E-NMPC is set to be 4 cycles in this study.

$$
\begin{gathered}
\tau_{\mathrm{sec}}=\frac{2 \cdot V_{\text {Man }}}{\eta_{V} \cdot V_{d} \cdot R P M_{E n g}} \\
\tau_{\text {cycle }}=\frac{V_{\text {Man }}}{\eta_{V} \cdot V_{d}}
\end{gathered}
$$




\subsection{Performance Assessment with SIL Co-simulation}

In this section, the performance of E-NMPC based engine control system are examined over two transient engine operating scenarios through the SIL co-simulation between SIMULINK and GT-SUITE. Two scenarios were duplicated in the engine dyno test cell by controlling the engine speeds and the pedal positions based on the recorded values during the vehicle tests. The production and instrumentation sensors showed in Figure 2.2 were used to record the engine data, and the recorded control actions were used as the baseline for comparing with E-NMPC based engine control results.

The first scenario is a transient test with intermittent ramps, which includes both speed/load transient and steady-state operating conditions. The engine operating conditions in both scenarios were recorded by the production engine controller during vehicle track tests. In the first transient test, there are five representative steady-state non-knocking engine operating conditions selected from the vehicle track tests, which covers from low speed/load conditions to high speed/load conditions. The first transient test cycle is used to check if the E-NMPC can quickly find the optimal set of control signals to achieve all three objectives, including tracking torque requests, minimizing fuel consumption, and reducing NOx emission. The second scenario is real-world vehicle driving tests, which include both gradual accelerations and decelerations of a vehicle and aggressive pedal tip-in/tip-out driving conditions. This driving test is used to examine the overall performances of the developed engine control system, including the torque tracking accuracy, specific fuel consumption and NOx emission over the entire drive cycle.

\subsubsection{Transient Test Assessment}

Figure 5.9 shows engine performance of the first transient test. The engine speed is from $1500 \mathrm{rpm}$ to $3500 \mathrm{rpm}$, and the IMEPn is from $250 \mathrm{kPa}$ to $840 \mathrm{kPa}$. It can be seen from Figure 5.9 that the E-NMPC based engine control system can smoothly and accurately 
track the target IMEPn without violating both the combustion stability constraint (COVIMEP) and the knock limit (KI). Under low speed/load conditions, between cycle 1 and 140, the E-NMPC can operate the engine near the combustion stability limit to minimize specific fuel consumption and decrease NOx emission. Over the entire test, the absolute errors of MAP prediction and IMEPn tracking are below $2 \mathrm{kPa}$ and $30 \mathrm{kPa}$, and the relative errors of above two terms are within $3 \%$ and $5 \%$. The RMSE errors of MAP prediction and IMEPn tracking are $0.5 \mathrm{kPa}$ and $10 \mathrm{kPa}$, respectively.

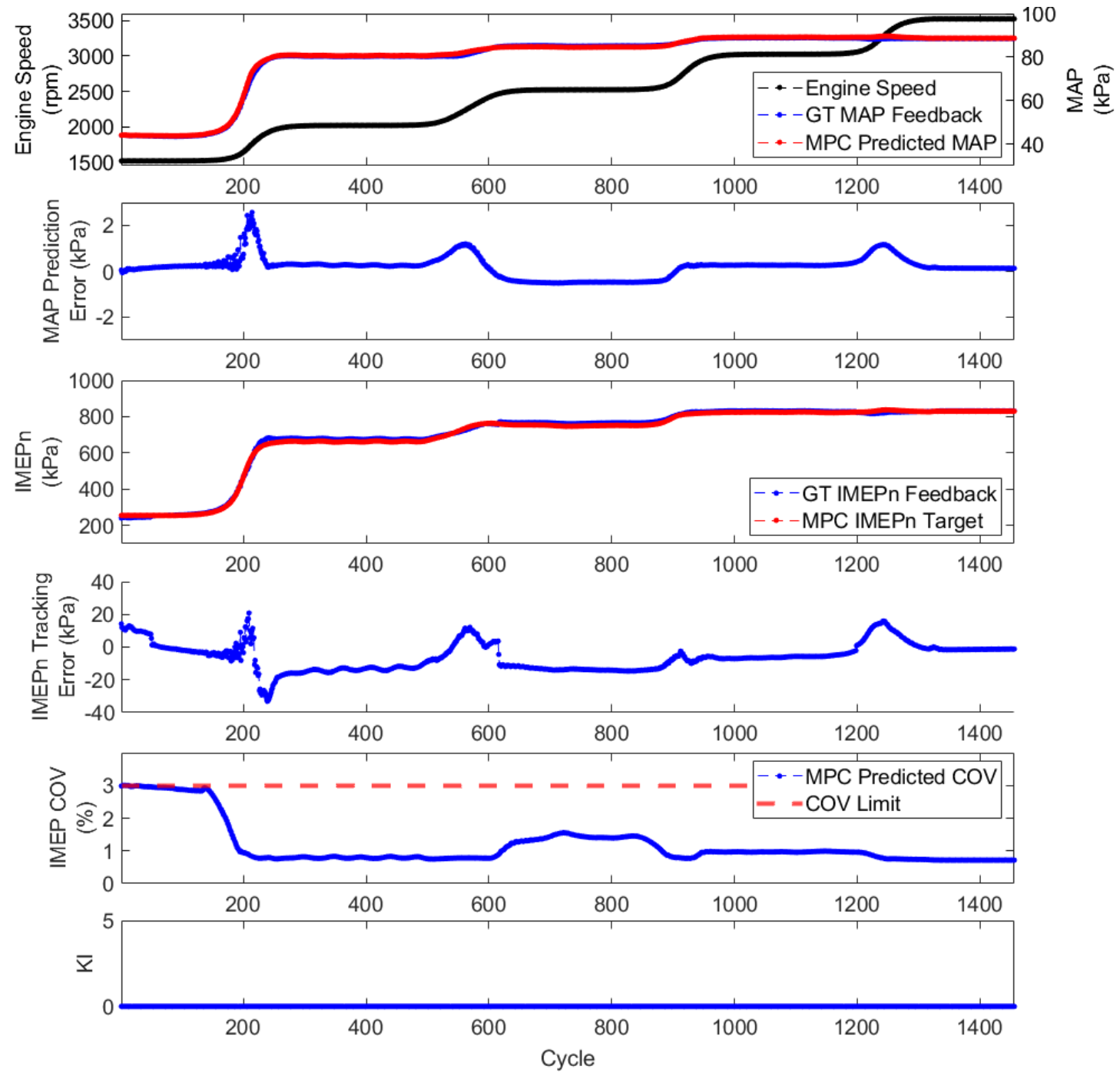

Figure 5.9 E-NMPC Engine Control Performances of the First Transient Test 
As showed in Figure 5.10, the control actions obtained by E-NMPC safely manipulate actuators with the consideration of the constraints listed in equation (5.37). The change rates of control signals considered in the cost function result in stable movement of controlled actuators. As indicated by 'GT Feedback CA50' in Figure 5.10, the adaptive spark timing management can track the optimal CA50 targets obtained from E-NMPC. The maximum absolute errors of CA50 tracking are below 1.5 CA degrees over the entire test and the RMSE error and the average error are 0.3 and $0.2 \mathrm{CA}$ degrees, respectively. The wastegate is opened to minimize the exhaust back pressure during the entire transient test, which helps to reduce the ISFC by decreasing the PMEP.
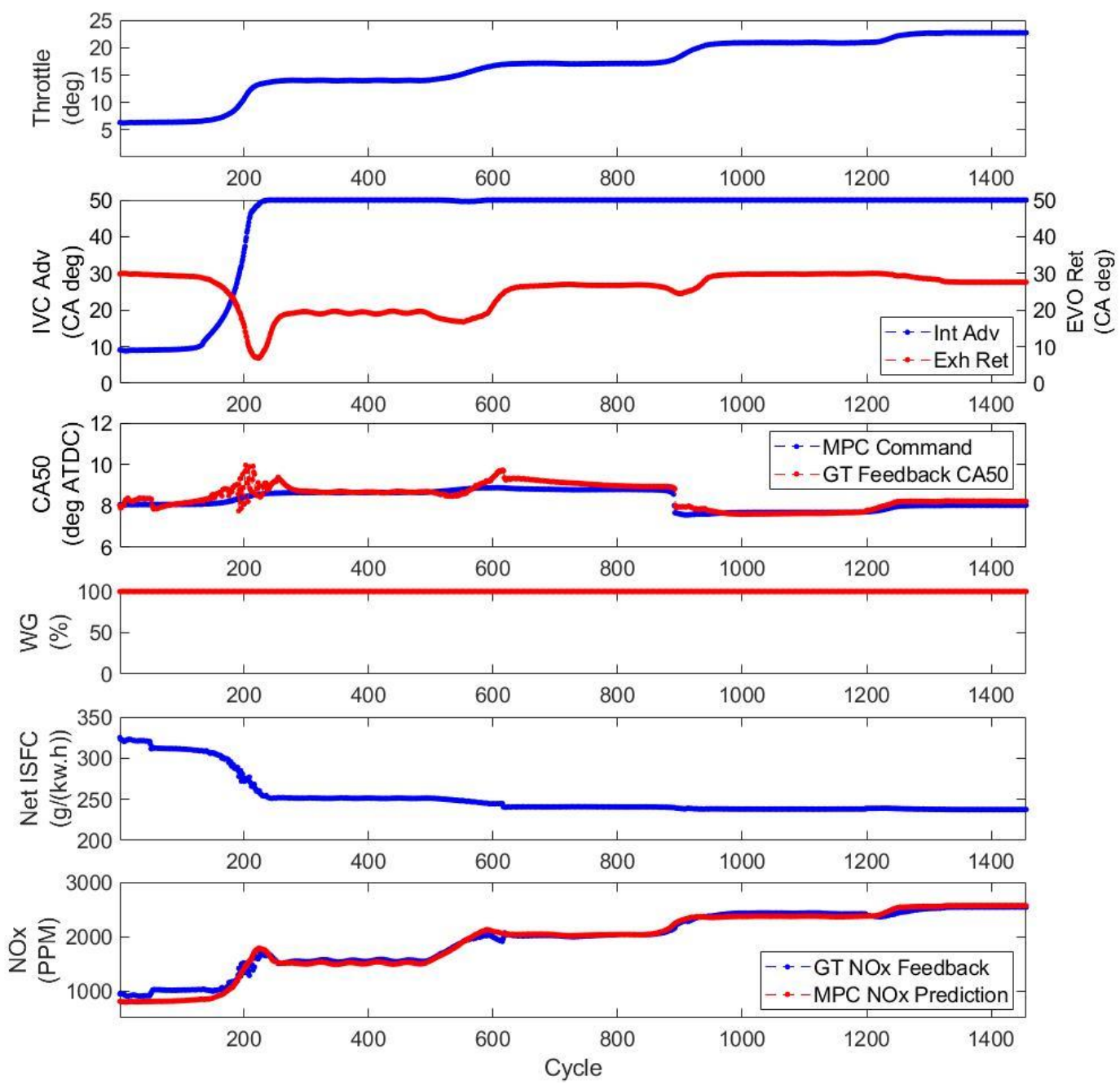

Figure 5.10 E-NMPC Engine Control Actions of the First Transient Test 


\subsubsection{Economic Performances Validation}

To evaluate the economic performance of the presented E-NMPC based engine control system, steady-state GT-POWER DOE simulations with sweeping of control actuators were conducted, which includes sweeping of CA50, intake cam timing, exhaust cam timing, and wastegate position. These DOE tests were used to examine if the control actions obtained by the E-NMPC can operate the engine within the best specific fuel consumption regions with minimum NOx emission.

\subsubsection{VVT Control Evaluation}

Figure 5.11 and Figure 5.12 are the contour plots of ISFC and cumulative NOx emission at EVO with sweeping of CAM timings, while CA50 was maintained at 8 deg ATDC. The red stars in Figure 5.11 and Figure 5.12 are average VVT control actions obtained from ENMPC under first transient operating condition. Comparing Figure 5.11 and Figure 5.12, it can be observed that the global minimum ISFC regions do not overlap with global minimum NOx areas. In Figure 5.12, the combinations of the earliest IVO and the latest EVC result in minimum NOx, where the RGF are maximized by the largest valve overlaps. Higher RGF leads to lower peak in-cylinder temperature, which reduces the NOx formation. However, for low speed/load condition, the best specific fuel consumption region (SFC) is located at the upper-left corner in Figure 5.11.(a), and this result agrees with the low speed/load experimental results conducted on 1.0L Ford engine [161]. In Figure 5.11.(a), although the late IVC deteriorates the ISFC with less effective compression ratio, retarding the EVO compensates this effect by increasing the effective expansion ratio. Late IVC also decreases PMEP by increasing the MAP through pushing gases back to the intake manifold, which increases the ISFC. Regarding higher speed/load conditions, as shown in Figure 5.11. (b) to Figure 5.11.(c), the best SFC are located at lower-right regions, and these results also agree with experimental data shown in [161]. The maximum IVC advance results in the highest effective compression ratio, and the proper EVO retard increases the effective expansion ratio. Under higher speed/load conditions, advancing IVC 
is more valuable than retarding EVO, which could be caused by less effective blow-down process. The GTDI SI engine is a dynamic system with nonlinear behaviors. Under certain speed and load conditions, the response surfaces of fuel consumption for variable valve timing may have multiple local minimum fuel consumption points [161]. It can be observed that Figure 5.11.(b) and (c) have multiple local optimal regions of the ISFC. The nonconvex response surface of fuel consumption can lead MPC to return nonoptimal solutions. In addition, MPC with multiple local optimal points may results in discontinuity of the controller. Based on above discussion, it is impossible to operate the engine within optimal regions to simultaneously achieve the minimum SFC and the minimum NOx by only including the single penalty of either fuel consumption or NOx emission in the cost function.

By incorporating both the fuel consumption penalty and the NOx emission penalty in equation (5.36), the proposed E-NMPC based engine control can derive the best achievable combinations of IVC and EVO to minimize the ISFC and reduce the NOx. Figure 5.11. (b) and (c) demonstrates that the developed E-NMPC can avoid nonoptimal solutions and operate the engine within achievable minimum fuel consumption regions. From Figure 5.10 to Figure 5.12, it can be seen that the E-NMPC can derive the continuous and smooth optimal VVT control actions during the speed/load transition periods. In this work, the weighting factors of fuel consumption and NOx emission were balanced in a way that the engine was operated within the minimum SFC region first. Then, ' $\mathrm{C}$ ' term in equation (5.36) was calibrated to reduce the NOx emission, till the engine was controlled near the borders of minimum ISFC regions. To adapt this E-NMPC based engine system to different control objectives and engine operating conditions, such as further reducing fuel consumption under part load or decreasing NOx emission under high engine load, adaptive techniques can be applied to have automated weightings on the fuel consumption penalty and NOx emission penalty based on engine operating conditions. 

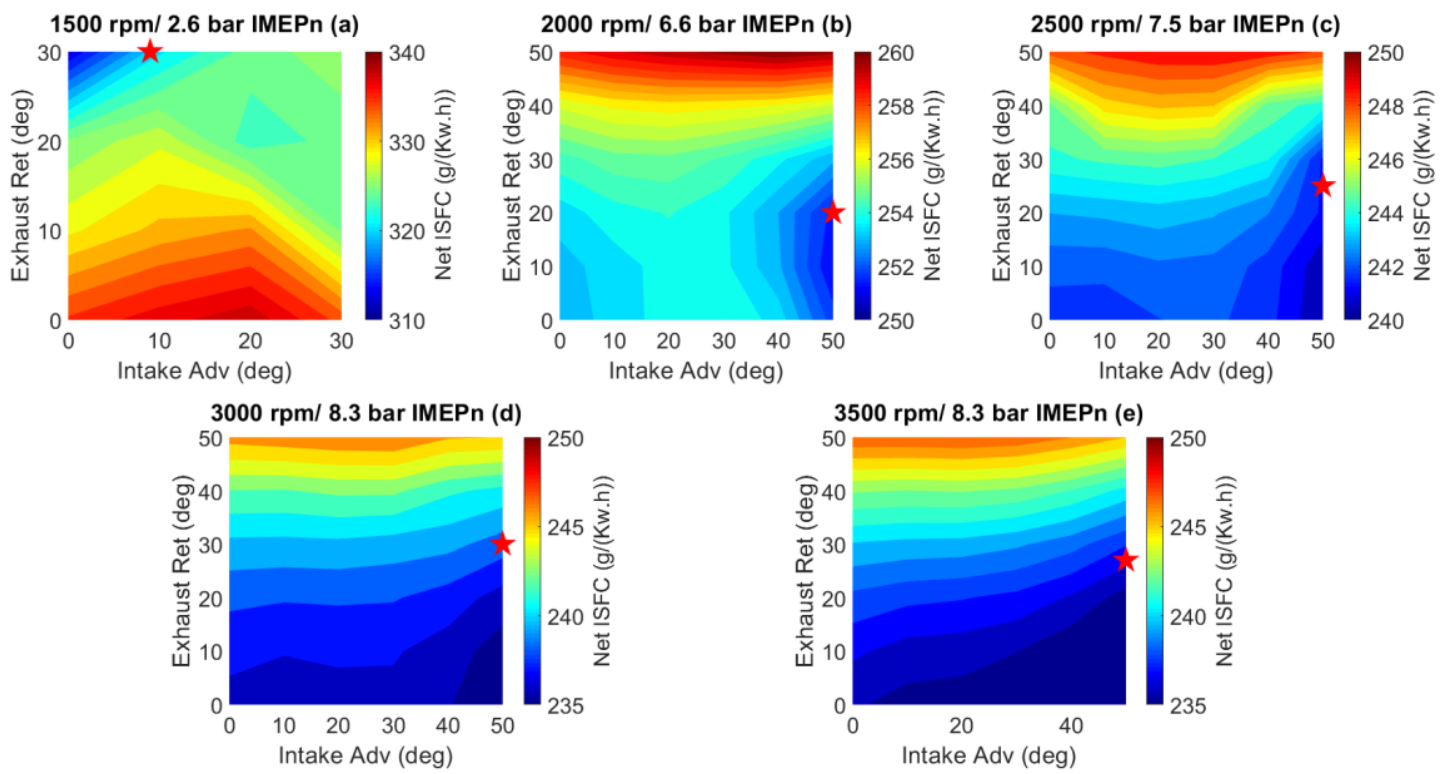

Figure 5.11 ISFC Results of GT-POWER DOE CAM Sweep Under Different Engine Speed and IMEPn with Fixed 8 degATDC CA50 and Wide Open Wastegate (Red stars are optimal CAM timings calculated by E-NMPC)
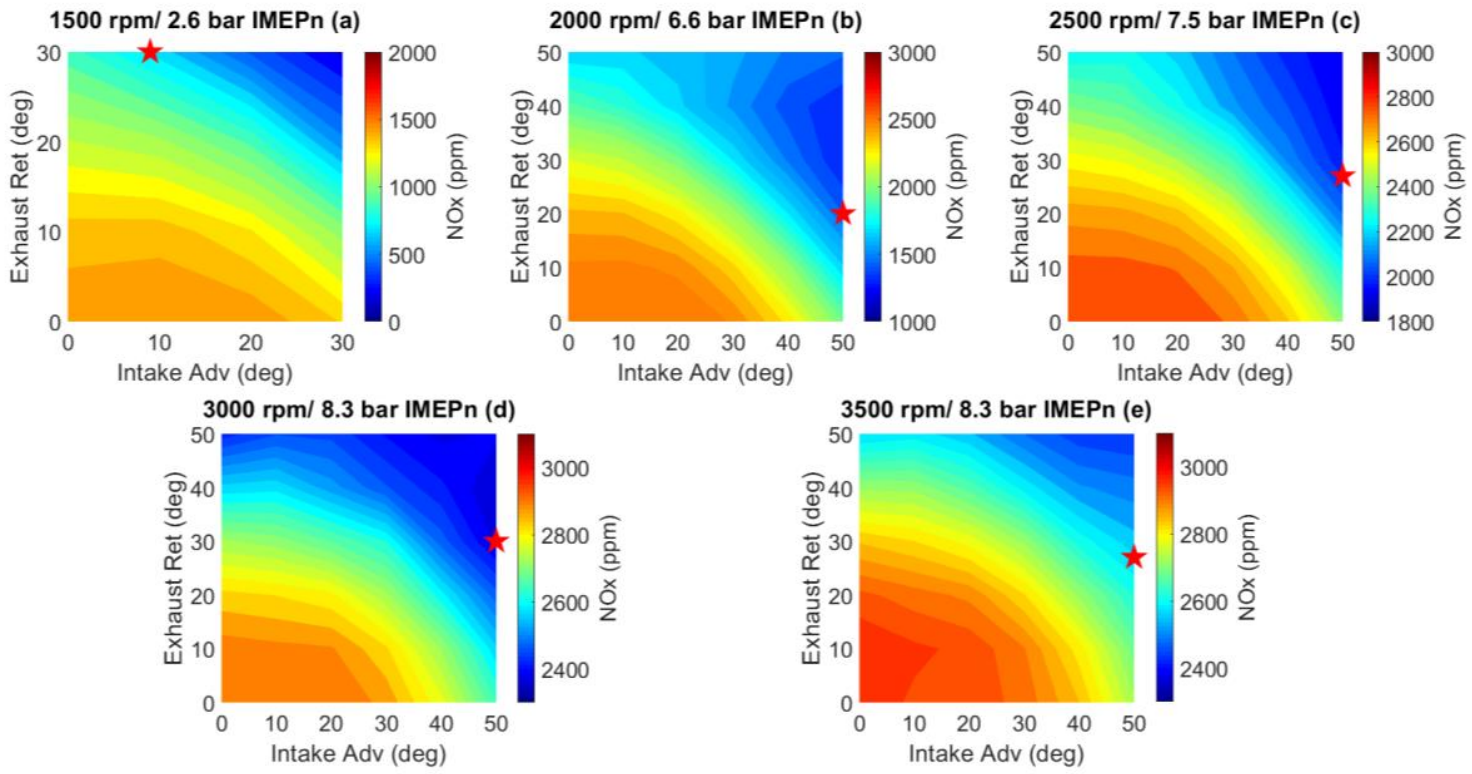

Figure 5.12 NOx Results of GT-POWER DOE CAM Sweep Under Different Engine Speed and IMEPn with Fixed 8 degATDC CA50 and 100\% Wastegate Opened (Red stars are optimal CAM timings calculated by E-NMPC) 


\subsubsection{CA50 Control Evaluation}

Figure 5.13 shows the CA50 sweep under two different speed/load conditions with fixed VVT timings. In both operating conditions, retarding the CA50 results in lower NOx emission, and this is due to the decreased peak in-cylinder temperature. When retards the CA50 from 8 deg ATDC to 10 deg ATDC, the NOx emission decreases $7.5 \%$ at $2000 \mathrm{rpm}$, and the NOx emission decreases $4.7 \%$ at $3000 \mathrm{rpm}$. Regarding the SFC, the optimal CA50 is $8 \mathrm{deg}$ ATDC at $2000 \mathrm{rpm}$. When the speed and load increases to $3000 \mathrm{rpm}$ and $830 \mathrm{kPa}$ IMEPn, the optimal CA50 is advanced to 6 deg ATDC. From Figure 5.13, it can be see that the ISFC increases $0.1 \%$ at $2000 \mathrm{rpm}$ and the ISFC increases $0.4 \%$ at $3000 \mathrm{rpm}$ when retards the CA50 from 8 deg ATDC to 10 deg ATDC. Figure 5.10 shows that the CA50 obtained by E-NMPC are continuously retarded when the engine speed and load are below $3000 \mathrm{rpm}$ and $830 \mathrm{kPa}$ IMEPn, which intends to decrease NOx and sacrifice a little bit SFC. The trends of the E-NMPC computed CA50 agree with the above discussion, because the ISFC is less sensitive to CA50 under lower engine speed and load operating conditions. The CA50 is advanced to optimal points at high engine speed and load operating conditions, this is because the fuel consumption penalty is more weighted than NOx emission penalty in equation (5.36) and the gradient of reducing ISFC is larger under higher engine speed and load operating conditions.
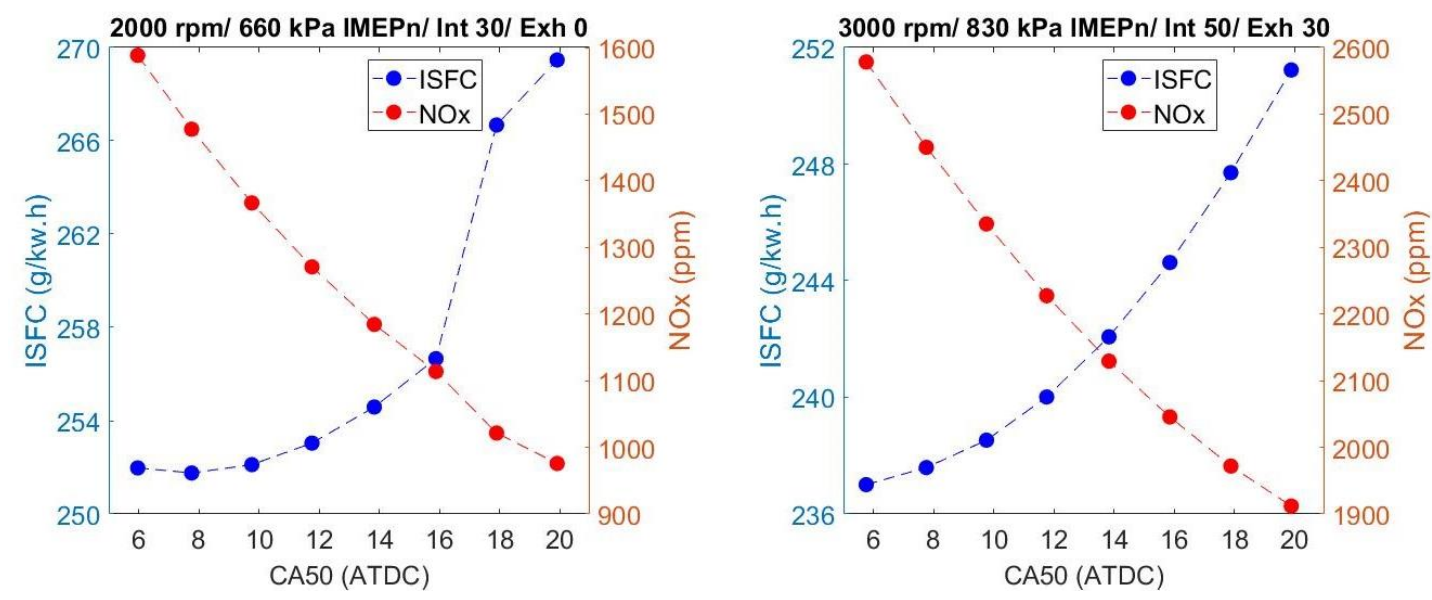

Figure 5.13 ISFC and NOx Results of GT-POWER DOE CA50 Sweep with 100\% Wastegate Opened 


\subsubsection{Real World Driving Cycle Assessment}

In order to examine the overall performances of E-NMPC engine control, a real-world driving cycle is used for SIL simulation. The process of fuel consumption and NOx emission comparison between the baseline engine control and the E-NMPC engine control is described in Figure 5.14. During on-road vehicle track test, the driver pedal position and engine speed are logged in the test vehicle. These logged values are fed to the dynamometer controller in the MTU APS test cell, and the test engine is controlled by the baseline engine controller to reproduce the engine operating conditions during the track test. During the dynamometer test, five control signals from the baseline engine control are recorded. To evaluate the fuel consumption and NOx emission of the baseline engine control, the above recorded control signals are fed to the GT-POWER engine model. The recorded engine speed and interpreted IMEPn are inputs of the E-NMPC engine controller. The E-NMPC controller issues the control actions to the GT-POWER engine model. The average ISFC and specific NOx emission described in equation (5.40) and (5.41) were used as the metrics for quantification and comparison of fuel consumption and NOx emission between the baseline engine control and E-NMPC engine control over the entire driving cycle. Equation (5.42) and (5.43) are used to quantify the differences of SFC and NOx emission between baseline engine control and E-NMPC engine control. 


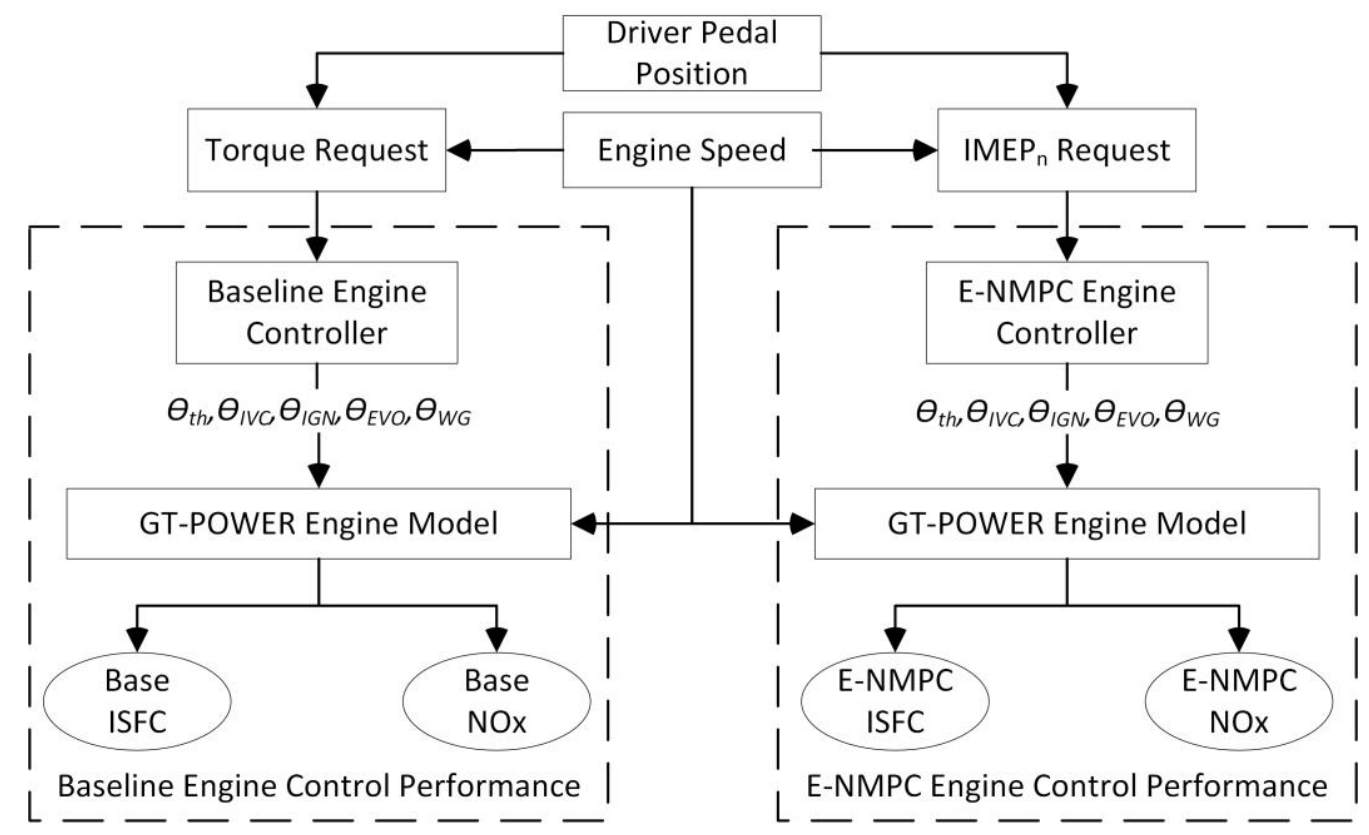

Figure 5.14 Process of Fuel Consumption and NOx Emission Comparison

$$
\begin{aligned}
& I S F C_{\text {Ave }}=\frac{\int_{t=0}^{t_{\text {cycle }}} \dot{m}_{f} \cdot d t}{\int_{t=0}^{t_{\text {cycle }}} P_{\text {Ind }} \cdot d t} \\
& N O x_{\text {Ave }}=\frac{\int_{t=0}^{t_{\text {cycle }}} Y_{N O x} \cdot \frac{M W_{N O_{2}}}{M W_{E x h}} \cdot \dot{m}_{E x h} \cdot d t}{\int_{t=0}^{t_{\text {cycle }}} P_{I n d} \cdot d t} \\
& I S F C_{\text {Ave_Diff }}=\frac{I S F C_{\text {Ave_Baseline }}-I S F C_{\text {Ave_NMPC }}}{I S F C_{\text {Ave_Baseline }}} \\
& N O x_{A v e_{-} \text {Diff }}=\frac{N O x_{A v e_{-} B a s e l i n e}-N O x_{A v e_{-} N M P C}}{N O x_{A v e_{-} B a s e l i n e}}
\end{aligned}
$$


Where,

$\begin{array}{ll}I S F C_{\text {Ave }} & \begin{array}{l}\text { Average indicated specific fuel consumption over the entire } \\ \text { driving cycle }(\mathrm{g} / \mathrm{KW} \cdot \mathrm{h})\end{array} \\ N O x_{\text {Ave }} & \text { Average NOx emission over the entire driving cycle }(\mathrm{g} / \mathrm{KW} \cdot \mathrm{h}) \\ t_{\text {cycle }} & \text { Total time of the driving cycle } \\ \dot{m}_{\text {fuel }} & \text { Fuel flow rate }(\mathrm{g} / \mathrm{s}) \\ P_{\text {Ind }} & \text { indicated power }(\mathrm{KW}) \\ M W & \text { Molecular weight }(\mathrm{g} / \mathrm{mol})\end{array}$

Figure 5.15 and Table 5.6 shows the speed/load map of the driving cycle test and details of engine acceleration/deceleration conditions. The transient engine speed profile was implemented in the GT-SUITE environment, and the E-NMPC receives the signal of IMEPn in the SIMULINK environment. The entire transient test takes 206 seconds and includes 2151 cycles of data.

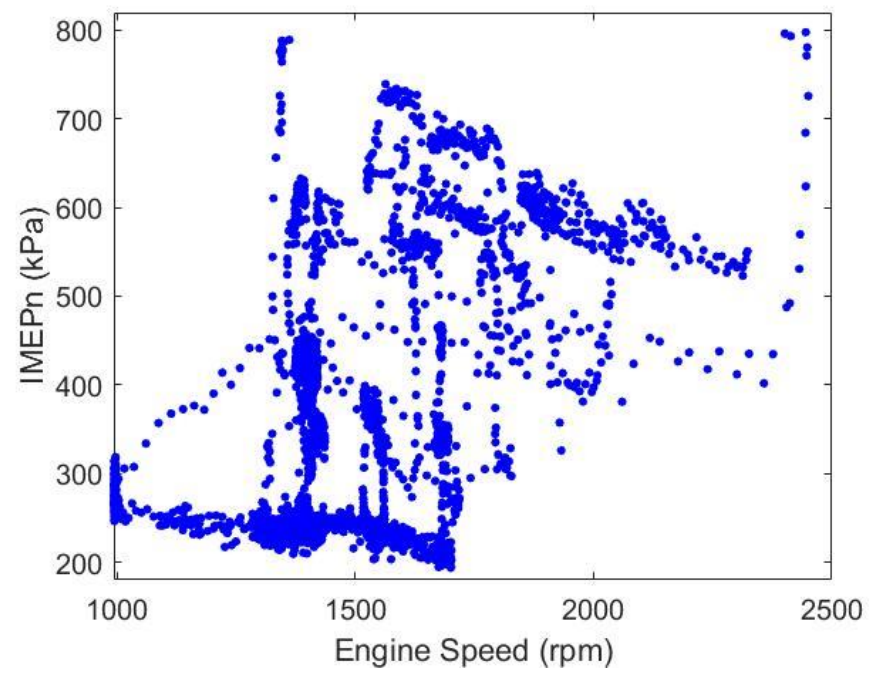

Figure 5.15 Dyno Test Engine Operating Points: Replicated from Real-Word Driving Cycle 
Table 5.6 Real-Word Driving Cycle Engine Acceleration/Deceleration Conditions

\begin{tabular}{|c|c|c|c|}
\hline $\begin{array}{c}\text { Max speed acceleration/deceleration } \\
(\mathbf{r p m} / \mathbf{s})\end{array}$ & \multicolumn{2}{c|}{$\begin{array}{c}\text { Max load (IMEPn) } \\
\text { acceleration/deceleration (bar/s) }\end{array}$} \\
\hline Acceleration & Deceleration & Acceleration & Deceleration \\
\hline 876 & -700 & 14.3 & -12.3 \\
\hline
\end{tabular}

Figure 5.16 and Figure 5.17 shows engine performances and E-NMPC control actions for the real-world driving test. Table 5.7 summarizes the performances of E-NMPC regarding IMEPn tracking and control-oriented engine model accuracies. From Figure 5.16 and Table 5.7, It can be observed that the E-NMPC based engine control system can track the target IMEPn with respecting both the combustion stability constraint (COVIMEP) and the knock limit (KI) during the entire driving test. For all pedal tip-in maneuvers, it can be seen that E-NMPC quickly advances IVC. This is because advancing IVC helps to trap enough air in the cylinder at higher engine speed and increase the peak in-cylinder pressure, which results in fast delivery of increased driver's torque request. For the tip-in events at low to medium engine speed/load conditions, like from cycle 400 to 500 and cycle 1400 to 1500 , EVO are retarded to increase the expansion work for fast increased load tracking. For pedal tip-out maneuvers, it can be observed that E-NMPC retards IVC, advances EVO, and immediately retards CA50 to decrease the engine torque output. Since spark timing is the fastest control actuation in the SI engine, sudden retard of CA50 helps to compensate for delay of intake manifold pressure dynamics during the tip-out event.

The third and fourth sub-plots in Figure 5.16 are the instantaneous ISFC and specific NOx emission. Compared to the baseline engine control, E-NMPC based engine control results in less fuel consumption and NOx emission over $84 \%$ and $99 \%$ of total cycles respectively. Table 5.8 summarizes the average fuel consumption and NOx emission over the entire driving cycle. E-NMPC based engine control reduces SFC by $1.9 \%$ and reduces the NOx emission by $47.9 \%$. Under low speed/load operating conditions, like from cycle 1 to 66, cycle 400 to 800 , and cycle 1163 to 1216 , E-NMPC manipulates the VVT to run the engine 
at COVIMEP limit by introducing longest valve overlap to achieve minimum SFC and reduce NOx emission. Under high engine speed/load operating conditions, like from cycle 142 to 245 and cycle 8602 to 960, E-NMPC issues the earliest IVC and appropriate EVO to reach the achievable operating points with minimum fuel consumption and NOx emission. The wastegate is widely opened under majority of the driving cycle to have minimum exhaust back pressure, which helps to reduce the SFC.

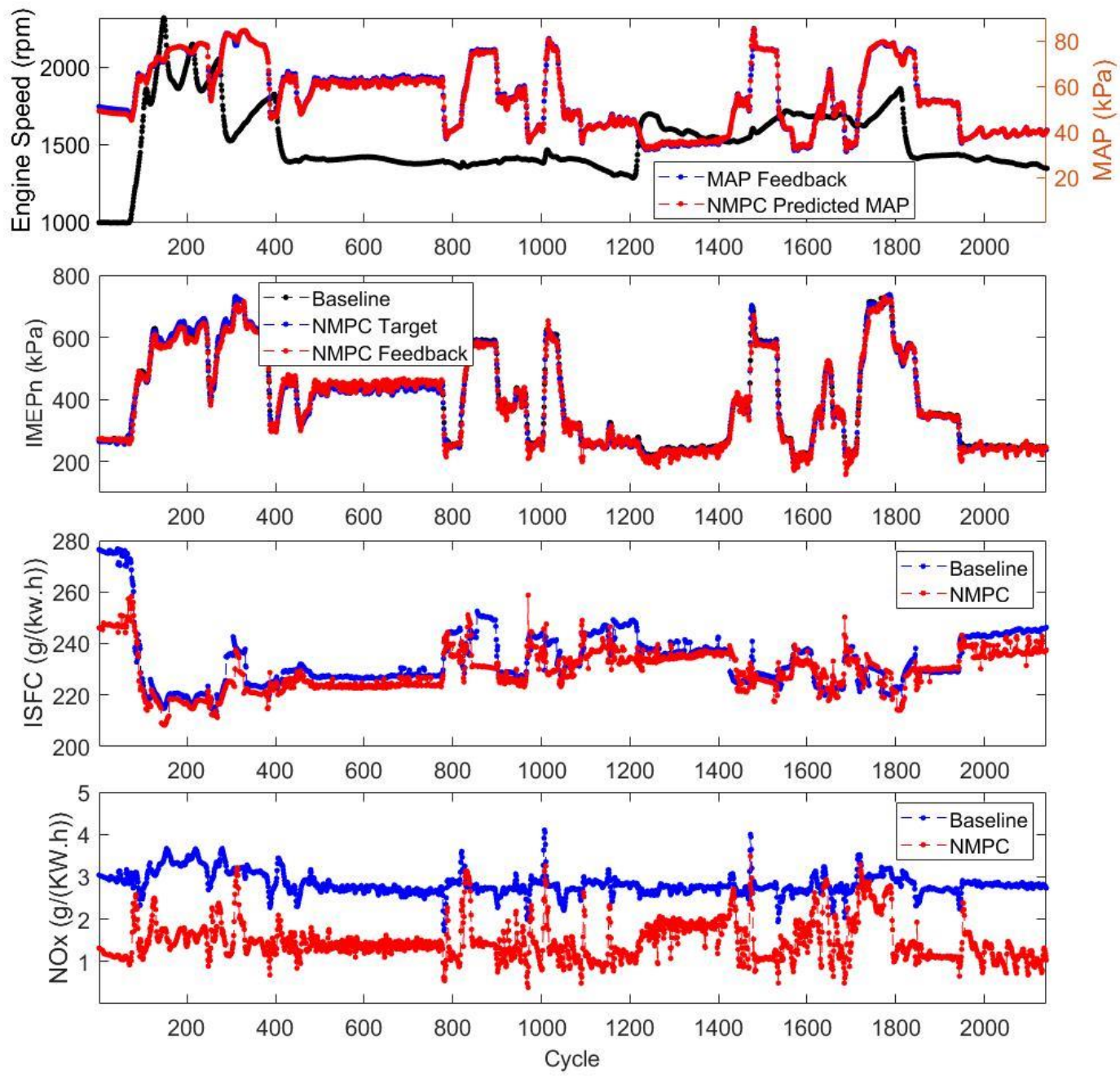

Figure 5.16 E-NMPC Engine Control Performances of the Real-Driving Cycle Test 

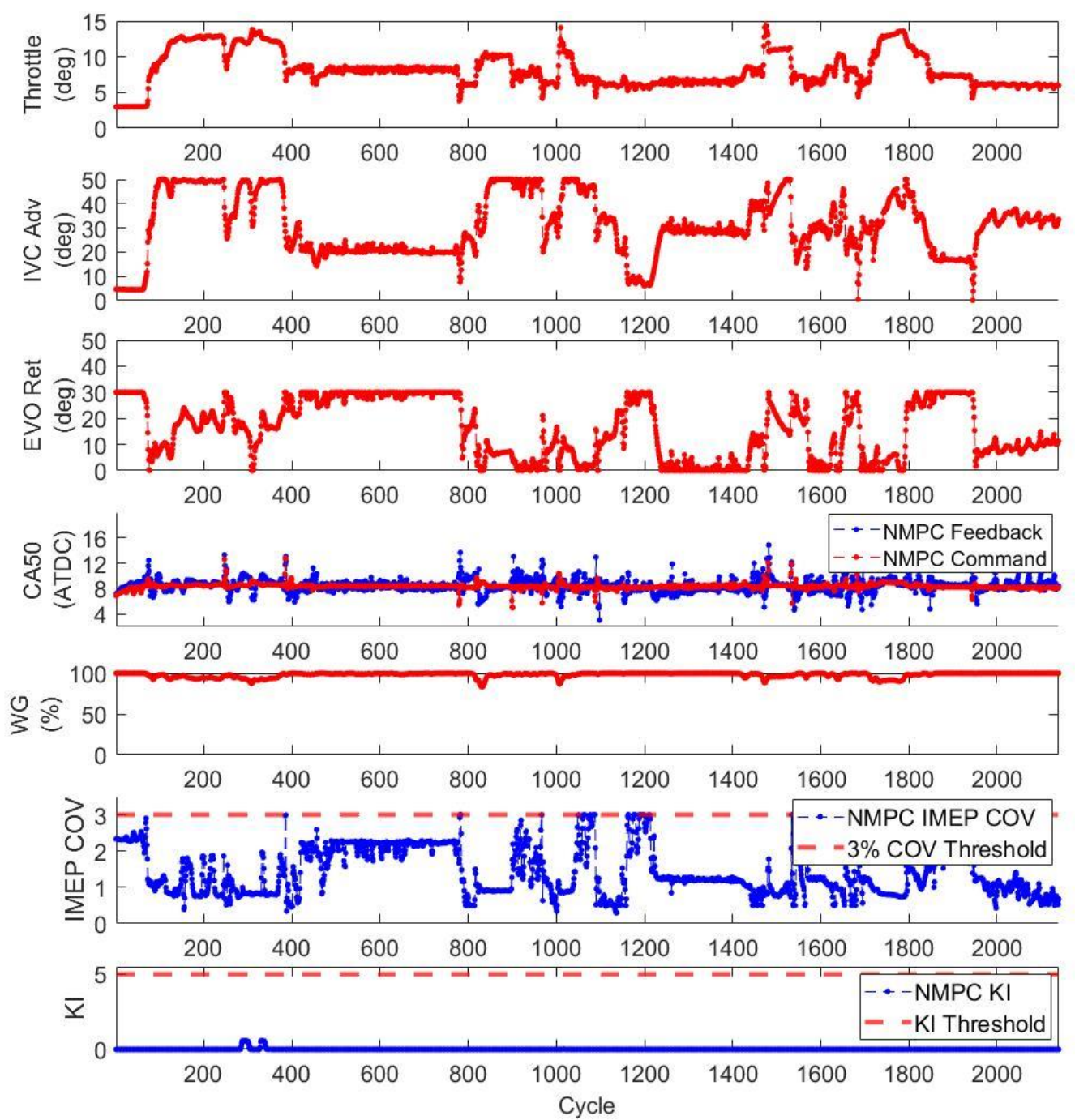

Figure 5.17 E-NMPC Engine Control Actions of the Real-Driving Cycle Test

Table 5.7 IMEPn Tracking and Control-Oriented Model Prediction Performance of ENMPC Engine Control

\begin{tabular}{|c|c|c|c|c|c|}
\hline & $\begin{array}{c}\text { IMEPn } \\
\text { Tracking } \\
\mathbf{( k P a )}\end{array}$ & $\begin{array}{c}\text { MAP } \\
\text { Prediction } \\
(\mathbf{k P a})\end{array}$ & $\begin{array}{c}\text { Boost } \\
\text { Pressure } \\
\text { Prediction } \\
\mathbf{( k P a )}\end{array}$ & $\begin{array}{c}\text { Volumetric } \\
\text { Efficiency } \\
\text { Prediction } \\
(\%)\end{array}$ & $\begin{array}{c}\text { EVO NOx } \\
\text { Prediction } \\
(\text { PPM) }\end{array}$ \\
\hline $\begin{array}{c}\text { Absolute } \\
\text { Mean } \\
\text { Error }\end{array}$ & 1.1 & 0.1 & 0.5 & 1.6 & 11 \\
\hline RMSE & 16.4 & 1.0 & 1.9 & 4.3 & 49 \\
\hline
\end{tabular}


Table 5.8 E-NMPC Engine Control vs. Baseline Engine Control

\begin{tabular}{|c|c|c|c|}
\hline \multicolumn{2}{|c|}{ ISFC } & \multicolumn{2}{|c|}{ NOxe $(\mathbf{g} / \mathbf{K W} \cdot \mathbf{h})$} \\
\hline Baseline & E-NMPC & Baseline & E-NMPC \\
\hline 232.3 & 228.0 & 2.90 & 1.51 \\
\hline \multicolumn{2}{|c|}{ ISFC $_{\text {Ave_Diff }}(\%)$} & \multicolumn{2}{|c|}{ NOx } \\
\hline \multicolumn{2}{|c|}{1.9} & \multicolumn{2}{c|}{48} \\
\hline
\end{tabular}

\subsection{Summary}

This chapter presents an E-NMPC based cycle-by-cycle engine control system for a GDTI SI engine. The presented E-NMPC aims to produce the requested IMEPn and simultaneously minimize the fuel consumption and NOx emission with consideration of a comprehensive set of engine operating constraints. To work with E-NMPC, a controloriented MIMO GTDI SI engine model has been developed by combining both physicsbased and neural-network based models. The E-NMPC model and control-oriented engine models were implemented in SIL environment using SIMULINK S-function blocks. The Adaptive Spark Timing Management model was implemented by a MATLAB function block in Simulink. The entire model-based engine control system was developed in MATLAB/SIMULINK and SIL testing was performed with a calibrated high-fidelity 1D GT-POWER engine model.

The performance of the E-NMPC engine control system was examined over two transient engine operating scenarios through the SIL simulation. In the first transient test, analysis has been conducted and the results demonstrate that the E-NMPC engine control system can stably and accurately track the driver's torque request and operate the engine within best achievable SFC regions and reduce the NOx emissions. In the real-world driving cycle test, E-NMPC based engine control is able to track the requested IMEPn with average absolute error of $1.1 \mathrm{kPa}$. Compared to baseline engine control, E-NMPC based engine control reduces the SFC by $1.9 \%$ and reduces the NOx emission by $48 \%$. In the future, the presented E-NMPC engine control system can be easily adapted to different engine operating scenarios and applications, such as the cold start, by modifying the weighting 
factors in the cost function and extending the control-oriented engine models to cover wider engine operating conditions. 


\section{Conclusions and Future Work}

\subsection{Conclusions}

In conclusion, this research develops a model-based cycle-by-cycle GDTI SI engine control system, which aims to deliver requested IMEPn, reduce the fuel consumption and NOx emissions with the consideration of engine operating constraints. Compared to traditional engine control system, the developed model-based engine control system can simultaneously achieve multiple objectives by systematically controlling all actuators, which has high potential to significantly reduce calibration efforts and shorten production cycle.

To achieve fast and accurate CA50 control, a framework of model-based feedforward ignition timing management using a computationally efficient control-oriented combustion model has been proposed. The combustion model uses only the information at and before the intake valve close (IVC) and considers the engine operating conditions. With only 64 calibration points, a RMSE of 1.7 degree and $\mathrm{R}^{2}$ of 0.95 shows the accuracy of the calibrated combustion model. On-road vehicle testing data is used to evaluate the performance of the developed model-based burn duration and ignition timing algorithm. From the transient tests, the developed combustion model is shown to cover wide engine operating conditions. When comparing the model predicted burn duration and ignition timing with experimental data, $83 \%$ of the prediction error falls within \pm 3 CAD. From the results of two different transient tests, the developed control-oriented combustion model has good interpolation/extrapolation ability and dynamic response. With the proper selection of the boundary calibration points, the control-oriented combustion model can be easily adapted to different engines, which contributes to shorten the engine development cycle. The dynamic burn duration and ignition timing prediction algorithm has been proposed to output the burn duration and the feedforward spark timing at IVC for the coming cycle based on the desired CA50. From the results of transient test, it can be observed that this algorithm only needs less than 5 iterations to predict the accurate burn duration based on the desired CA50 target. The algorithm can be easily implemented into 
the engine ECU for the real-time combustion phasing control without heavy computational burden.

In the fourth chapter, an RLS-VFF based adaptation algorithm is developed to handle operating-point-dependent model errors, which helps to maintain the accuracy of combustion model and ignition timing prediction throughout the engine lifetime and further reduces the need of calibration. A novel variable forgetting factor considers both cyclic combustion variations and base model errors is integrated into the RLS algorithm, which avoids fault adaptation and assists the adaptation algorithm to be stable. The developed RLS-VFF based adaptation is able to expand the working ranges of the original modelbased feedforward ignition timing management and cover the entire engine operating conditions without additional calibration efforts. From the transient test, the developed VFF-RLS-based model adaptation technique helps to reduce average prediction error of burn duration from 1.5 CAD to $0.1 \mathrm{CAD}$, and the average ignition timing prediction error is reduced from 1.4 CAD to 0 CAD. Since $99 \%$ of cycles converge within 3 iterations, it shows the capability of online application. Based on the stochastic characteristics of cycleto-cycle combustion variation, a CA50 observer is proposed using an adaptive Extended Kalman Filter and developed combustion model. The AEKF-based feedback CA50 estimation has been evaluated using heavy transient test and the results show the accurate and fast estimation of CA50 under transient operating conditions. Compared to traditional CA50 estimation using a moving average method, the AEKF algorithm successfully 'filters' out the 'stochastic noise' and provides 'filtered' CA50 estimation without significant cycle delays.

In the fifth chapter, a model-based control system for cycle-by-cycle control of a GTDI SI engine using an economic nonlinear model predictive controller has been developed to simultaneously achieve three objectives: instantaneous delivery of requested IMEPn, minimization of fuel consumption, and reduction of NOx emissions. A control-oriented MIMO GTDI SI engine model has been developed to predict the future engine behaviors by incorporating both physics-based and data-driven modeling approaches. The controloriented MIMO engine model is calibrated using DOE simulation results from a high- 
fidelity 1D GT-POWER engine model, which has been calibrated and validated by experimental engine data. The performance of the E-NMPC engine control system has been evaluated through the SIL co-simulation between SIMULINK and GT-POWER using two transient tests. The first transient test demonstrates that the developed E-NMPC engine control system can track the IMEPn targets within 3\% error and operate the engine within the best achievable minimum fuel and NOx emission regions through coordinating VVT, ignition timings, and wastegate opening percentages. The second transient test is used to quantify the overall performance of entire model-based engine control system. Over the entire driving cycle, the average error of IMEPn tracking is $5 \mathrm{kPa}$. Compared to baseline engine control, the proposed model-based engine control system reduces the SFC by $1.9 \%$ and reduces the NOx emission by $48 \%$. The E-NMPC based engine control system can be easily adapted to different engine design and future upgrading by recalibrating system models used in MPC. Adaptive techniques can be applied to introduce adaptive weightings on the fuel consumption penalty and NOx emission penalty to achieve different control objectives based on engine operating conditions. 


\subsection{Future Work}

This research work provides a framework of the model-based control for cycle-by-cycle GTDI SI engine control. The future work is suggested as follows:

- Since the in-cylinder pressure sensor is costly, modify the model-based feedforward ignition timing management for combustion phasing control by eliminating in-cylinder pressure sensors.

- The E-NMPC based engine control system is developed and calibrated for normal engine operating conditions after the engine warmup stage. The adaptation method can be integrated to expand the working ranges of the control system, such as cold start.

- Knock control in a SI engine is a stochastic process and has many uncontrolled noise factors that change the onset of knock including effective fuel anti-knock index (AKI), deposits, humidity, intake air temperature, etc. Therefore, in addition to feedforward combustion knock prediction, a closed-loop knock controller is needed to control SI combustion knock effectively.

- The E-NMPC based engine control system can be utilized for assistance/assessment of production calibration and tuning for steady-state and transient engine control.

- Implement E-NMPC based engine control system in prototyping system for further development, verification, and validation. 


\section{$7 \quad$ Reference List}

1. Agency, I.E., World Energy Outlook 2019. 2019.

2. National Highway Traffic Safety Administration, U.S.E.P.A.E. March 31, 2020; Available from: https://www.nhtsa.gov/press-releases/safe-final-rule.

3. Agency, U.S.E.P., Proposed Rule for Technical Amendments to the Light-duty Vehicle Greenhouse Gas Program, U.S.E.P. Agency, Editor. 2018.

4. Yamaji, K., M. Tomimatsu, I. Takagi, A. Higuchi, T. Yoshida, and E. Murase, New 2.OL I4 Gasoline Direct Injection Engine with Toyota New Global Architecture Concept. 2018, SAE International.

5. Chen, W., C. Xia, K. Mao, J. Tao, J. Fang, and Z. Huang, The Effects of Injection Strategies on Particulate Emissions from a Dual-Injection Gasoline Engine. 2019, SAE International.

6. Osorio, J.D. and A. Rivera-Alvarez, Efficiency enhancement of spark-ignition engines using a Continuous Variable Valve Timing system for load control. Energy, 2018. 161: p. 649-662.

7. hakariya, M., T. Toda, and M. Sakai, The New Toyota Inline 4-Cylinder 2.5L Gasoline Engine. 2017, SAE International.

8. Yamazaki, D., A. Mori, and E. Murase, The Development of a New V6 3.5L Turbocharged Gasoline Engine, in SAE Technical Paper Series. 2018, SAE International.

9. Shibata, M., M. kawamata, H. Komatsu, K. Maeyama, M. Asari, N. Hotta, K. Nakada, and H. Daicho, New 1.OL I3 Turbocharged Gasoline Direct Injection Engine. 2017, SAE International.

10. Kargul, J., M. Stuhldreher, D. Barba, C. Schenk, S. Bohac, J. McDonald, P. Dekraker, and J. Alden, Benchmarking a 2018 Toyota Camry 2.5-Liter Atkinson Cycle Engine with Cooled-EGR. 2019, SAE International.

11. Zhang, D., Q. Lu, M. Kocsis, I. Gilbert, M. Megel, X. Liu, J. Gu, Q. Liu, and Y. He, The New BAIC High Efficiency Turbocharged Engine with LPL-EGR. 2017, SAE International.

12. Kojima, S., S. Kiga, K. Moteki, E. Takahashi, and K. Matsuoka, Development of a New $2 L$ Gasoline VC-Turbo Engine with the World's First Variable Compression Ratio Technology, in SAE Technical Paper Series. 2018, SAE International. 
13. Teodosio, L., V. De Bellis, F. Bozza, and D. Tufano, Numerical Study of the Potential of a Variable Compression Ratio Concept Applied to a Downsized Turbocharged VVA Spark Ignition Engine. 2017, SAE International.

14. Bosch GmbH, R., Gasoline Engine Management. 2015.

15. Anjum, R., A. Yar, I.K. Yousufzai, Q. Ahmed, and A.I. Bhatti. Second Order Sliding Mode based Speed Tracking Control for Torque Management of Gasoline Engines. in 2019 12th Asian Control Conference (ASCC). 2019.

16. Triwiyatno, A., M. Nuh, A. Santoso, and I. Sutantra, Engine Torque Control of Spark Ignition Engine using Fuzzy Gain Scheduling. TELKOMNIKA (Telecommunication Computing Electronics and Control), 2012. 10.

17. Kolyubin, S., D. Efimov, V. Nikiforov, and A. Bobtsov, Two-channel adaptive hybrid control of the air-to-fuel ratio and torque of automobile engines. Automation and Remote Control, 2012. 73.

18. Jaiprakash and G. Habib, On-road assessment of light duty vehicles in Delhi city: Emission factors of CO, CO2 and NOX. Atmospheric Environment, 2018. 174: p. 132-139.

19. Wu, B., R.G. Prucka, Z. Filipi, D.M. Kramer, and G.L. Ohl, Cam-phasing Optimization Using Artificial Neural Networks as Surrogate Models-Fuel Consumption and NOx Emissions. 2006, SAE International.

20. Pourkhesalian, A.M., A.H. Shamekhi, and F. Salimi, NOx Control Using Variable Exhaust Valve Timing and Duration. 2010, SAE International.

21. Corre, S., B. Mason, T. Steffen, E. Winward, Z. Yang, T. Childs, M. Cary, and R. Lygoe, Application of Multi-Objective Optimization Techniques for Improved Emissions and Fuel Economy over Transient Manoeuvres. 2019.

22. Yang, Z., E. Winward, B. Mason, S.L. Corre, T. Childs, and A. Shahzad. Nonlinear Model Predictive Control of a Variable Valve Timing System in a Turbocharged Spark Ignition Engine*. in 2019 American Control Conference (ACC). 2019.

23. Cyclic dispersion in engine combustion-Introduction by the special issue editors. International Journal of Engine Research, 2015. 16(3): p. 255-259.

24. Luo, W., B. Chen, J. Naber, and C. Glugla, Stochastic Knock Detection, Control, Software Integration, and Evaluation on a V6 Spark-Ignition Engine under Steady-State Operation. 2014, SAE International. 
25. Rassweiler, G.M. and L. Withrow, Motion Pictures of Engine Flames Correlated with Pressure Cards. 1938, SAE International.

26. Heywood, J.B., Internal combustion engine fundamentals. 2018.

27. Zhang, B., C.W. Ji, and S.F. Wang, Combustion analysis and emissions characteristics of a hydrogen-blended methanol engine at various spark timings. International Journal of Hydrogen Energy, 2015. 40(13): p. 4707-4716.

28. Miganakallu, N., Z. Yang, R. Rogóż, Ł.J. Kapusta, C. Christensen, S. Barros, and J. Naber, Effect of water - methanol blends on engine performance at borderline knock conditions in gasoline direct injection engines. Applied Energy, 2020. 264: p. 114750.

29. Rodriguez, J.F. and W.K. Cheng, Analysis of NOx Emissions during Crank-Start and Cold Fast-Idle in a GDI Engine. 2017, SAE International.

30. Liu, Y., J. Deng, Z. Hu, and L. Li, In-cycle combustion feedback control for abnormal combustion based on digital ion current signal. International Journal of Engine Research, 2017. 19(2): p. 241-249.

31. Advanced Combustion Engines and Fuels, U.S.D.o. Energy, Editor. 2019.

32. Wang, Y., J. Zhang, Z. Yang, X. Wang, P. Dice, M. Shahbakhti, J. Naber, M. Czekala, Q. Qu, and G. Huberts, Investigation of Flow Conditions and Tumble near the Spark Plug in a DI Optical Engine at Ignition. 2018, SAE International.

33. Gao, J. and T. Shen, Cylinder pressure sensor-based real-time combustion phase control approach for SI engines. IEEJ Transactions on Electrical \& Electronic Engineering, 2017. 12(2): p. 244-250.

34. Maurya, R.K., Closed-Loop Combustion Control, in Characteristics and Control of Low Temperature Combustion Engines: Employing Gasoline, Ethanol and Methanol. 2018, Springer International Publishing: Cham. p. 483-510.

35. Cavina, N., N. Rojo, A. Businaro, and R. Cevolani, Comparison between Pressure- and Ion-Current-Based Closed-Loop Combustion Control Performance. 2019, SAE International.

36. Malaczynski, G., G. Roth, and D. Johnson, Ion-Sense-Based Real-Time Combustion Sensing for Closed Loop Engine Control. 2013, SAE International.

37. Zhang, Y., X. Shen, and T. Shen, A survey on online learning and optimization for spark advance control of SI engines. Science China Information Sciences, 2018. 61(7): p. 70201. 
38. Gao, J., Y. Zhang, and T. Shen, An On-Board Calibration Scheme for Map-Based Combustion Phase Control of Spark-Ignition Engines. IEEE/ASME Transactions on Mechatronics, 2017. 22(4): p. 1485-1496.

39. Grasreiner, S., J. Neumann, M. Wensing, and C. Hasse, Model-based virtual engine calibration with the help of phenomenological methods for spark-ignited engines. Applied Thermal Engineering, 2017. 121: p. 190-199.

40. Xiang, L., E. Song, and Y. Ding, A Two-Zone Combustion Model for Knocking Prediction of Marine Natural Gas SI Engines. Energies, 2018. 11(3): p. 561.

41. Khoa, N.X. and O. Lim, The effects of combustion duration on residual gas, effective release energy, engine power and engine emissions characteristics of the motorcycle engine. Applied Energy, 2019. 248: p. 54-63.

42. Xiao, B., S. Wang, and R.G. Prucka, An investigation of semiphysical artificial neural networks for multi-fuel combustion phasing control of spark ignition engines. Proceedings of the Institution of Mechanical Engineers, Part D: Journal of Automobile Engineering, 2014. 228(11): p. 1262-1273.

43. Johnson, R., D. Kaczynski, W. Zeng, A. Warey, R. Grover, and S. Keum, Prediction of Combustion Phasing Using Deep Convolutional Neural Networks. 2020, SAE International.

44. Blizard, N.C. and J.C. Keck, Experimental and Theoretical Investigation of Turbulent Burning Model for Internal Combustion Engines. 1974, SAE International.

45. Wang, S., R. Prucka, Q. Zhu, M. Prucka, and H. Dourra, A Real-Time Model for Spark Ignition Engine Combustion Phasing Prediction. SAE International Journal of Engines, 2016. 9(2).

46. Zhu, Q., R. Prucka, S. Wang, M. Prucka, and H. Dourra, Model-Based Optimal Combustion Phasing Control Strategy for Spark Ignition Engines. SAE International Journal of Engines, 2016. 9(2): p. 1170-1179.

47. Wang, S., Q. Zhu, R. Prucka, M. Prucka, and H. Dourra, Input Adaptation for Control Oriented Physics-Based SI Engine Combustion Models Based on Cylinder Pressure Feedback. SAE International Journal of Engines, 2015. 8(4).

48. Ling, C.H. and M.A. Abas, One-dimensional simulation using port water injection for a spark ignition engine. International Journal of Automotive and Mechanical Engineering, 2018. 15: p. 5803-5814. 
49. De Bellis, V., F. Bozza, S. Fontanesi, E. Severi, and F. Berni, Development of a Phenomenological Turbulence Model through a Hierarchical 1D/3D Approach Applied to a VVA Turbocharged Engine. 2016, SAE International.

50. Bozza, F., V. De Bellis, F. Berni, A. D'Adamo, and L. Maresca, Refinement of a OD Turbulence Model to Predict Tumble and Turbulent Intensity in SI Engines. Part I: 3D Analyses. 2018, SAE International.

51. Hires, S.D., R.J. Tabaczynski, and J.M. Novak, The Prediction of Ignition Delay and Combustion Intervals for a Homogeneous Charge, Spark Ignition Engine, in SAE Technical Paper Series. 1978, SAE International.

52. Martin, J.K., S.L. Plee, and D.J. Remboski, Burn Modes and Prior-Cycle Effects on Cyclic Variations in Lean-Burn Spark-Ignition Engine Combustion. 1988, SAE International.

53. Lindström, F., Elmqvist, G. Kalghatgi, and A. H-E, An Empirical SI Combustion Model Using Laminar Burning Velocity Correlations. Vol. 144. 2005.

54. Qu, Z., M. Ma, and F. Zhao, An Online Crank-Angle-Resolved Mean-Value Combustion Model of Gasoline Engines Including Effects of Cycle Initial States. 2012, SAE International.

55. Yeliana, Parametric combustion modeling for ethanol-gasoline fuelled spark ignition engines. 2010.

56. Bane, S.P.M., J.L. Ziegler, and J.E. Shepherd. Development of One-Step Chemistry Models for Flame and Ignition Simulation. 2010.

57. Qin, S.J. and T.A. Badgwell, A survey of industrial model predictive control technology. Control Engineering Practice, 2003. 11(7): p. 733-764.

58. Ding, Y., L. Wang, Y. Li, and D. Li, Model predictive control and its application in agriculture: A review. Computers and Electronics in Agriculture, 2018. 151: p. 104-117.

59. Lozano Santamaría, F. and J.M. Gómez, An Algorithm for Tuning NMPC Controllers with Application to Chemical Processes. Industrial \& Engineering Chemistry Research, 2016. 55(34): p. 9215-9228.

60. Mejía, J.A.P., L.A. Silva, and J.A.P. Flórez. Control Strategy for Oil Production Wells with Electrical Submersible Pumping Based on the Nonlinear Model-Based Predictive Control Technique. in 2018 IEEE ANDESCON. 2018. 
61. Gros, S., R. Quirynen, and M. Diehl. Aircraft control based on fast non-linear MPC \& multiple-shooting. in 2012 IEEE 51 st IEEE Conference on Decision and Control (CDC). 2012.

62. Swief, A., A. El-Zawawi, and M. El-Habrouk. A Survey of Model Predictive Control Development in Automotive Industries. in 2019 International Conference on Applied Automation and Industrial Diagnostics (ICAAID). 2019.

63. Bromnick, P., Development of a Model Predictive Controller for Engine Idle Speed using CPower. 1999, SAE International.

64. Bemporad, A., D. Bernardini, R. Long, and J. Verdejo, Model Predictive Control of Turbocharged Gasoline Engines for Mass Production, in SAE Technical Paper Series. 2018.

65. Zhu, Q., S. Onori, and R. Prucka, An Economic Nonlinear Model Predictive Control Strategy for SI Engines: Model-Based Design and Real-Time Experimental Validation. IEEE Transactions on Control Systems Technology, 2018: p. 1-15.

66. Pizzonia, F., T. Castiglione, and S. Bova, A Robust Model Predictive Control for efficient thermal management of internal combustion engines. Applied Energy, 2016. 169: p. 555-566.

67. Bemporad, A., D. Bernardini, M. Livshiz, and B. Pattipati, Supervisory Model Predictive Control of a Powertrain with a Continuously Variable Transmission. 2018, SAE International.

68. Hatanaka, T., T. Yamada, M. Fujita, S. Morimoto, and M. Okamoto, Explicit Receding Horizon Control of Automobiles with Continuously Variable Transmissions, in Nonlinear Model Predictive Control: Towards New Challenging Applications, L. Magni, D.M. Raimondo, and F. Allgöwer, Editors. 2009, Springer Berlin Heidelberg: Berlin, Heidelberg. p. 561-569.

69. Carneiro, J.S.A. and L. Ferrarini, Preventing Thermal Overloads in Transmission Circuits via Model Predictive Control. IEEE Transactions on Control Systems Technology, 2010. 18(6): p. 1406-1412.

70. Bordons, C., M.A. Ridao, A. Pérez, A. Arce, and D. Marcos. Model Predictive Control for power management in hybrid fuel cell vehicles. in 2010 IEEE Vehicle Power and Propulsion Conference. 2010.

71. Hu, X., C. Zou, X. Tang, T. Liu, and L. Hu, Cost-Optimal Energy Management of Hybrid Electric Vehicles Using Fuel Cell/Battery Health-Aware Predictive Control. IEEE Transactions on Power Electronics, 2020. 35(1): p. 382-392. 
72. Golchoubian, P. and N.L. Azad, Real-Time Nonlinear Model Predictive Control of a Battery-Supercapacitor Hybrid Energy Storage System in Electric Vehicles. IEEE Transactions on Vehicular Technology, 2017. 66(11): p. 9678-9688.

73. Ripaccioli, G., D. Bernardini, S.D. Cairano, A. Bemporad, and I.V. Kolmanovsky. A stochastic model predictive control approach for series hybrid electric vehicle power management. in Proceedings of the 2010 American Control Conference. 2010.

74. Wang, Y., X. Wang, Y. Sun, and S. You, Model predictive control strategy for energy optimization of series-parallel hybrid electric vehicle. Journal of Cleaner Production, 2018. 199: p. 348-358.

75. Fu, J., S. Song, Z. Fu, and J. Ma, Hierarchical Model Predictive Control for Parallel Hybrid Electrical Vehicles. Asian Journal of Control, 2018. 20(6): p. 2331-2342.

76. Huang, Y., H. Wang, A. Khajepour, H. He, and J. Ji, Model predictive control power management strategies for HEVs: A review. Journal of Power Sources, 2017. 341: p. 91-106.

77. Cheng, M. and B. Chen, Nonlinear Model Predictive Control of a Power-Split Hybrid Electric Vehicle With Consideration of Battery Aging. Journal of Dynamic Systems, Measurement, and Control, 2019. 141(8).

78. Wang, H., J. Oncken, and B. Chen. Receding Horizon Control for Mode Selection and Powertrain Control of a Multi-mode Hybrid Electric Vehicle. in 2019 IEEE 90th Vehicular Technology Conference (VTC2019-Fall). 2019.

79. Oncken, J.E. and B. Chen, Real-Time Model Predictive Powertrain Control for a Connected Plug-In Hybrid Electric Vehicle. IEEE Transactions on Vehicular Technology, 2020: p. 1-1.

80. Oncken, J., J. Orlando, P.K. Bhat, B. Narodzonek, C. Morgan, D. Robinette, B. Chen, and J. Naber, A Connected Controls and Optimization System for Vehicle Dynamics and Powertrain Operation on a Light-Duty Plug-In Multi-Mode Hybrid Electric Vehicle. 2020, SAE International.

81. Moser, D., R. Schmied, H. Waschl, and L.d. Re, Flexible Spacing Adaptive Cruise Control Using Stochastic Model Predictive Control. IEEE Transactions on Control Systems Technology, 2018. 26(1): p. 114-127.

82. Naus, G.J.L., J. Ploeg, M.J.G. Van de Molengraft, W.P.M.H. Heemels, and M. Steinbuch, Design and implementation of parameterized adaptive cruise control: An explicit model predictive control approach. Control Engineering Practice, 2010. 18(8): p. 882-892. 
83. Tang, L., F. Yan, B. Zou, K. Wang, and C. Lv, An Improved Kinematic Model Predictive Control for High-Speed Path Tracking of Autonomous Vehicles. IEEE Access, 2020. 8: p. 51400-51413.

84. Barik, B., P.K. Bhat, J. Oncken, B. Chen, J. Orlando, and D. Robinette, Optimal velocity prediction for fuel economy improvement of connected vehicles. IET Intelligent Transport Systems, 2018. 12(10): p. 1329-1335.

85. Murilo, A., R. Rodrigues, E.L.S. Teixeira, and M.M.D. Santos, Design of a Parameterized Model Predictive Control for Electric Power Assisted Steering. Control Engineering Practice, 2019. 90: p. 331-341.

86. Xu, S., H. Peng, P. Lu, M. Zhu, and Y. Tang, Design and Experiments of Safeguard Protected Preview Lane Keeping Control for Autonomous Vehicles. IEEE Access, 2020. 8: p. 29944-29953.

87. Cheng, S., L. Li, H. Guo, Z. Chen, and P. Song, Longitudinal Collision Avoidance and Lateral Stability Adaptive Control System Based on MPC of Autonomous Vehicles. IEEE Transactions on Intelligent Transportation Systems, 2020. 21(6): p. 2376-2385.

88. Baharuddin, M., K. Kassim, and M.R.A. Mansor, Autonomous Emergency Brake (AEB) for Pedestrian for ASEAN NCAP Safety Rating Consideration: A Review. 2019. 3: p. 63-73.

89. Herceg, M., T. Raff, R. Findeisen, and F. Allgowe. Nonlinear model predictive control of a turbocharged diesel engine. in 2006 IEEE Conference on Computer Aided Control System Design, 2006 IEEE International Conference on Control Applications, 2006 IEEE International Symposium on Intelligent Control. 2006.

90. Ortner, P. and L.d. Re, Predictive Control of a Diesel Engine Air Path. IEEE Transactions on Control Systems Technology, 2007. 15(3): p. 449-456.

91. Huang, M., H. Nakada, K. Butts, and I. Kolmanovsky, Nonlinear Model Predictive Control of a Diesel Engine Air Path: A Comparison of Constraint Handling and Computational Strategies. IFAC-PapersOnLine, 2015. 48(23): p. 372-379.

92. Hadef, J.E., S. Olaru, P. Rodriguez-Ayerbe, G. Colin, Y. Chamaillard, and V. Talon. Explicit nonlinear model predictive control of the air path of a turbocharged spark-ignited engine. in 2013 IEEE International Conference on Control Applications (CCA). 2013.

93. Zhu, Q., R. Koli, L. Feng, S. Onori, and R. Prucka. Nonlinear model predictive air path control for turbocharged SI engines with low pressure EGR and a continuous surge valve. in 2017 American Control Conference (ACC). 2017. 
94. Hu, Y., H. Chen, P. Wang, H. Chen, and L. Ren, Nonlinear model predictive controller design based on learning model for turbocharged gasoline engine of passenger vehicle. Mechanical Systems and Signal Processing, 2018. 109: p. 7488.

95. Keller, M., S. Geiger, M. Günther, S. Pischinger, D. Abel, and T. Albin, Model predictive air path control for a two-stage turbocharged spark-ignition engine with low pressure exhaust gas recirculation. International Journal of Engine Research, 2020: p. 1468087420936398.

96. Zhu, Q., R. Prucka, M. Prucka, and H. Dourra, A Nonlinear Model Predictive Control Strategy with a Disturbance Observer for Spark Ignition Engines with External EGR. SAE International Journal of Commercial Vehicles, 2017. 10(1): p. $360-372$.

97. Kang, M. and T. Shen. Modeling and optimal control for torque tracking of spark-ignition engines with low pumping loss. in 2016 35th Chinese Control Conference (CCC). 2016.

98. Xin, Z., L. You, H. Yunfeng, and C. Hong. Torque tracking control of turbocharged gasoline engine using nonlinear MPC. in 2015 European Control Conference (ECC). 2015.

99. Kang, M. and T. Shen. Experimental comparisons between LQR and MPC for spark-ignition engine control problem. in 2017 36th Chinese Control Conference (CCC). 2017.

100. Lee, T.-K. and Z. Filipi, Nonlinear Model Predictive Control of Advanced Engines Using Discretized Nonlinear Control Oriented Models. 2010, SAE International.

101. Grüne, L.P., Jürgen, Nonlinear Model Predictive Control: Theory and Algorithms. 2017, Springer: Springer.

102. Yang, L., Y. Huang, M. Xia, and H. Li, Neural-network based boost pressure prediction for two-stage turbocharging system of diesel engine. IFACPapersOnLine, 2019. 52(5): p. 178-184.

103. Egan, D., R. Koli, Q. Zhu, and R. Prucka, Use of Machine Learning for RealTime Non-Linear Model Predictive Engine Control. 2019, SAE International.

104. Irdmousa, B.K., S.Z. Rizvi, J.M. Veini, J.D. Nabert, and M. Shahbakhti. Datadriven Modeling and Predictive Control of Combustion Phasing for RCCI Engines. in 2019 American Control Conference (ACC). 2019. 
105. Kang, M. and T. Shen. Model predictive control for automotive gasoline engines. in 2017 13th IEEE International Conference on Control \& Automation (ICCA). 2017.

106. Zhu, Q., S. Onori, and R. Prucka. Nonlinear economic Model Predictive Control for SI engines based on Sequential Quadratic Programming. in 2016 American Control Conference (ACC). 2016.

107. Koli, R., D. Egan, Q. Zhu, and R. Prucka, Quantification of Linear Approximation Error for Model Predictive Control of Spark-Ignited Turbocharged Engines. 2019, SAE International.

108. Wang, X., A. Khameneian, P. Dice, B. Chen, M. Shahbakhti, J.D. Naber, C. Archer, Q. Qu, C. Glugla, and G. Huberts. Model-Based Combustion Duration and Ignition Timing Prediction for Combustion Phasing Control of a SparkIgnition Engine Using In-Cylinder Pressure Sensors. in ASME 2019 International Design Engineering Technical Conferences and Computers and Information in Engineering Conference. 2019.

109. Yazdani, A., J. Naber, M. Shahbakhti, P. Dice, C. Glugla, S. Cooper, D. McEwan, and G. Huberts, Air Charge and Residual Gas Fraction Estimation for a SparkIgnition Engine Using In-Cylinder Pressure. 2017, SAE International.

110. Medina, A., P.L. Curto-Risso, A.C. Hernández, L. Guzmán-Vargas, F. AnguloBrown, and A.K. Sen, Quasi-dimensional simulation of spark ignition engines. 2014: Springer.

111. Bonatesta, F., B. Waters, and P.J. Shayler, Burn angles and form factors for Wiebe function fits to mass fraction burned curves of a spark ignition engine with variable valve timing. International Journal of Engine Research, 2009. 11(2): p. 177-186.

112. Tabaczynski, R.J., F.H. Trinker, and B.A.S. Shannon, Further refinement and validation of a turbulent flame propagation model for spark-ignition engines. Combustion and Flame, 1980. 39(2): p. 111-121.

113. van Tiggelen, A. and J. Deckers, Chain branching and flame propagation. Symposium (International) on Combustion, 1957. 6(1): p. 61-66.

114. Wang, S., R. Prucka, M. Prucka, and H. Dourra, Control-oriented residual gas mass prediction for spark ignition engines. International Journal of Engine Research, 2014. 16(7): p. 897-907.

115. Asmus, T.W., Valve Events and Engine Operation. 1982, SAE International. 
116. Khameneian, A., X. Wang, P. Dice, B. Chen, M. Shahbakhti, J.D. Naber, C. Archer, Q. Qu, C. Glugla, and G. Huberts. MODEL-BASED DYNAMIC INCYLINDER AIR CHARGE, RESIDUAL GAS AND TEMPERATURE ESTIMATION FOR A GDI SPARK IGNITION ENGINE USING CYLINDER, INTAKE AND EXHAUST PRESSURES. in ASME 2020 Dynamic Systems and Control Conference. 2020.

117. Toulson, E., A. Huisjen, X. Chen, C. Squibb, G. Zhu, H. Schock, and W.P. Attard, Visualization of Propane and Natural Gas Spark Ignition and Turbulent Jet Ignition Combustion. 2012, SAE International.

118. Irimescu, A., S.S. Merola, and G. Valentino, Application of an entrainment turbulent combustion model with validation based on the distribution of chemical species in an optical spark ignition engine. Applied Energy, 2016. 162: p. 908923.

119. Robinet, C. and P. Higelin, Crossed Study of Residual Gas Rate - Firing Device for a Better Understanding of SI Engines Cycle-to-Cycle Variations. 1998, SAE International.

120. Ozdor, N., M. Dulger, and E. Sher, Cyclic Variability in Spark Ignition Engines A Literature Survey. 1994, SAE International.

121. Elisei-Iliescu, C., C. Paleologu, and R. Tamaş. On the performance of variable forgetting factor recursive least-squares algorithms. in Advanced Topics in Optoelectronics, Microelectronics, and Nanotechnologies 2016. 2016. SPIE.

122. Fortescue, T.R., L.S. Kershenbaum, and B.E. Ydstie, Implementation of selftuning regulators with variable forgetting factors. Automatica, 1981. 17(6): $\mathrm{p}$. 831-835.

123. Paleologu, C., J. Benesty, and S. Ciochina, A Robust Variable Forgetting Factor Recursive Least-Squares Algorithm for System Identification. IEEE Signal Processing Letters, 2008. 15: p. 597-600.

124. Agrell, F., H.-E. Ångström, B. Eriksson, J. Wikander, and J. Linderyd, Transient Control of HCCI Combustion by aid of Variable Valve Timing Through the use of a Engine State Corrected CA50-Controller Combined with an In-Cylinder State Estimator Estimating Lambda. 2005, SAE International.

125. Zhu, Q., S. Wang, R. Prucka, M. Prucka, and H. Dourra, Model-Based ControlOriented Combustion Phasing Feedback for Fast CA50 Estimation. SAE International Journal of Engines, 2015. 8(3). 
126. Raut, A., B.K. Irdmousa, and M. Shahbakhti, Dynamic modeling and model predictive control of an RCCI engine. Control Engineering Practice, 2018. 81: p. 129-144.

127. Ravi, N., H.-H. Liao, A.F. Jungkunz, A. Widd, and J.C. Gerdes, Model predictive control of HCCI using variable valve actuation and fuel injection. Control Engineering Practice, 2012. 20(4): p. 421-430.

128. Akhlaghi, S., N. Zhou, and Z. Huang, Adaptive Adjustment of Noise Covariance in Kalman Filter for Dynamic State Estimation. 2017.

129. Almagbile, A., J. Wang, and W. Ding, Evaluating the performances of adaptive Kalman filter methods in GPS/INS integration. Journal of Global Positioning Systems, 2010. 9(1): p. 33-40.

130. Raghupatruni, I., S. Burton, M. Boumans, T. Huber, and A. Reiter, Credibility of software-in-the-loop environments for integrated vehicle function validation. 2020, Springer Fachmedien Wiesbaden: Wiesbaden. p. 299-313.

131. Ranuzzi, F., N. Cavina, A. Brusa, M. De Cesare, and M. Panciroli, Development and Software in the Loop Validation of a Model-based Water Injection Combustion Controller for a GDI TC Engine. 2019, SAE International.

132. Singh, K. and M. Arat, Deep Learning in the Automotive Industry: Recent Advances and Application Examples. 2019.

133. Ashok, B., S. Denis Ashok, and C. Ramesh Kumar, A review on control system architecture of a SI engine management system. Annual Reviews in Control, 2016. 41: p. 94-118.

134. Turkson, R.F., F. Yan, M.K.A. Ali, and J. Hu, Artificial neural network applications in the calibration of spark-ignition engines: An overview. Engineering Science and Technology, an International Journal, 2016. 19(3): p. 1346-1359.

135. MacKay, D.J.C., Bayesian Interpolation. Neural Computation, 1992. 4(3): p. 415447.

136. Foresee, F.D. and M.T. Hagan. Gauss-Newton approximation to Bayesian learning. in Proceedings of International Conference on Neural Networks (ICNN'97). 1997.

137. Magner, S., M. Jankovic, and S. Cooper, Methods to Reduce Air-charge Characterization Data for High Degree of Freedom Engines. 2004, SAE International. 
138. Peyton Jones, J.C. and K.R. Muske, Identification and adaptation of linear lookup table parameters using an efficient recursive least-squares technique. ISA Transactions, 2009. 48(4): p. 476-483.

139. de Nola, F., G. Giardiello, A. Gimelli, A. Molteni, M. Muccillo, and R. Picariello, Volumetric efficiency estimation based on neural networks to reduce the experimental effort in engine base calibration. Fuel, 2019. 244: p. 31-39.

140. El Hadef, J., G. Colin, V. Talon, and Y. Chamaillard, Neural Model for Real-Time Engine Volumetric Efficiency Estimation. 2013, SAE International.

141. Malaczynski, G.W., M. Mueller, J. Pfeiffer, D. Cabush, and K. Hoyer, Replacing Volumetric Efficiency Calibration Look-up Tables with Artificial Neural Networkbased Algorithm for Variable Valve Actuation. 2010, SAE International.

142. Kocher, L., E. Koeberlein, D. Alstine, K. Stricker, and G. Shaver, PhysicallyBased Volumetric Efficiency Model for Diesel Engines Utilizing Variable Intake Valve Actuation. International Journal of Engine Research - INT J ENGINE RES, 2012. 13: p. 169-184.

143. Albin, T., D. Ritter, N. Liberda, R. Quirynen, and M. Diehl, In-Vehicle Realization of Nonlinear MPC for Gasoline Two-Stage Turbocharging Airpath Control. IEEE Transactions on Control Systems Technology, 2018. 26(5): p. 1606-1618.

144. Koli, R., H. Arunachalam, Q. Zhu, S. Onori, A. Vahidi, and R. Prucka. Nonlinear Model Predictive Control of Dual Loop - Exhaust Gas Recirculation in a Turbocharged Spark Ignited engine. in 2018 Annual American Control Conference (ACC). 2018.

145. Caton, J.A., Combustion phasing for maximum efficiency for conventional and high efficiency engines. Energy Conversion and Management, 2014. 77: p. 564576.

146. Teodosio, L., D. Pirrello, F. Berni, V. De Bellis, R. Lanzafame, and A. D'Adamo, Impact of intake valve strategies on fuel consumption and knock tendency of a spark ignition engine. Applied Energy, 2018. 216: p. 91-104.

147. Yuan, Z., J. Liu, J. Fu, Q. Liu, S. Wang, and Y. Xia, Quantitative analysis on the thermodynamics processes of gasoline engine and correction of the control equations for heat-work conversion efficiency. Energy Conversion and Management, 2017. 132: p. 388-399.

148. Liu, Q., J. Fu, G. Zhu, Q. Li, J. Liu, X. Duan, and Q. Guo, Comparative study on thermodynamics, combustion and emissions of turbocharged gasoline direct 
injection (GDI) engine under NEDC and steady-state conditions. Energy

Conversion and Management, 2018. 169: p. 111-123.

149. Naber, J.D., J.R. Blough, D. Frankowski, M. Goble, and J.E. Szpytman, Analysis of Combustion Knock Metrics in Spark-ignition Engines. SAE Transactions, 2006. 115: p. 223-243.

150. Li, T., T. Yin, and B. Wang, A phenomenological model of knock intensity in spark-ignition engines. Energy Conversion and Management, 2017. 148: p. 12331247.

151. Alam, A., M. Mittal, and V. Lakshminarasimhan, Analysis of In-Cylinder Flow and Cycle-to-Cycle Flow Variations in a Small Spark-Ignition Engine at Different Throttle Openings. 2020, SAE International.

152. Di Mauro, A., H. Chen, and V. Sick, Neural network prediction of cycle-to-cycle power variability in a spark-ignited internal combustion engine. Proceedings of the Combustion Institute, 2019. 37(4): p. 4937-4944.

153. Zhu, Q., R. Prucka, S. Wang, M. Prucka, and H. Dourra. Control oriented modelling of engine IMEP variation. in ASME 2016 Internal Combustion Engine Division Fall Technical Conference. 2016. American Society of Mechanical Engineers Digital Collection.

154. Galloni, E., Analyses about parameters that affect cyclic variation in a spark ignition engine. Applied Thermal Engineering, 2009. 29(5): p. 1131-1137.

155. Lee, T.K., R.G. Prucka, and Z.S. Filipi, Real-time estimation of combustion variability for model-based control and optimal calibration of spark ignition engines. Proceedings of the Institution of Mechanical Engineers, Part D: Journal of Automobile Engineering, 2009. 223(11): p. 1361-1372.

156. Durand, H., M. Ellis, and P.D. Christofides, Economic model predictive control designs for input rate-of-change constraint handling and guaranteed economic performance. Computers \& Chemical Engineering, 2016. 92: p. 18-36.

157. Gros, S., M. Zanon, R. Quirynen, A. Bemporad, and M. Diehl, From linear to nonlinear MPC: bridging the gap via the real-time iteration. International Journal of Control, 2016: p. 1-19.

158. Houska, B., H.J. Ferreau, and M. Diehl, An auto-generated real-time iteration algorithm for nonlinear MPC in the microsecond range. Automatica, 2011. 47(10): p. 2279-2285. 
159. Quirynen, R., M. Vukov, M. Zanon, and M. Diehl, Autogenerating microsecond solvers for nonlinear MPC: A tutorial using ACADO integrators. Optimal Control Applications and Methods, 2015. 36(5): p. 685-704.

160. Ferreau, H.J., C. Kirches, A. Potschka, H.G. Bock, and M. Diehl, qpOASES: a parametric active-set algorithm for quadratic programming. Mathematical Programming Computation, 2014. 6(4): p. 327-363.

161. Bonatesta, F., G. Altamore, J. Kalsi, and M. Cary, Fuel economy analysis of partload variable camshaft timing strategies in two modern small-capacity spark ignition engines. Applied Energy, 2016. 164: p. 475-491. 


\title{
Appendix A: Copyright Documentation
}

\author{
Permission to use the entire peer-reviewed paper from ASME
}

$8 / 11 / 2020$

Michigan Technological University Mail - Permission Request for ASME 2019 IDETC Conference Paper No. IDETC2019-97703

Michigan Tech

Xin Wang <xwang15@mtu.edu>

Permission Request for ASME 2019 IDETC Conference Paper No. IDETC2019-97703

Beth Darchi <DarchiB@asme.org>

To: Xin Wang <xwang15@mtu.edu>

Thu, Jul 23, 2020 at 1:58 PM

Dear Mr. Wang,

This permission has been revised. It is our pleasure to grant you permission to use all or any part of the following ASME papers:

- Control-Oriented Model-Based Burn Duration and Ignition Timing Prediction With RecursiveLeast-Square Adaptation for Closed-Loop Combustion Phasing Control of a Spark Ignition Engine, by Xin Wang, Amir Khameneian, Paul Dice, Bo Chen, Mahdi Shahbakhti, Jeffrey D. Naber, Chad Archer, Quuping Qu, Chris Glugla, Garlan Huberts, Paper No: DSCC2019-9073

- Model-Based Combustion Duration and Ignition Timing Prediction for Combustion Phasing Control of a Spark-Ignition Engine Using In-Cylinder Pressure Sensors, by Xin Wang, Amir Khameneian, Paul Dice, Bo Chen, Mahdi Shahbakhti, Jeffrey D. Naber, Chad Archer, Qiuping Qu, Chris Glugla, Garlan Huberts, Paper No: DETC2019-97703

cited in your letter for inclusion in a dissertation tentatively entitled A STUDY OF MODEL-BASED CONTROL STRATEGY FOR A GASOLINE TURBOCHARGED DIRECT INJECTION SPARK IGNITED ENGINE to be published by Michigan Technological University.

Permission is granted for the specific use as stated herein and does not permit further use of the materials without proper authorization. Proper attribution must be made to the author(s) of the materials. Please note: if any or all of the figures and/or Tables are of another source, permission should be granted from that outside source or include the reference of the original source. ASME does not grant permission for outside source material that may be referenced in the ASME works.

As is customary, we request that you ensure full acknowledgment of this material, the author(s), source and ASME as original publisher.

Many thanks for your interest in ASME publications.

Sincerely,

\section{Beth Darchi}


8/11/2020 Michigan Technological University Mail - Permission Request for ASME 2019 IDETC Conference Paper No. IDETC2019-97703

Publishing Administrator

ASME

2 Park Avenue

New York, NY 10016-5990

Tel 1.212.591.7700

darchib@asme.org

\begin{abstract}
From: Xin Wang <xwang15@mtu edu>
Sent: Monday, July 20, 2020 2:15 PM
\end{abstract}

[Quoted text hidden]

[Quoted text hidden] 